\title{
Set Identified Linear Models
}

\section{CHRISTIAN BONTEMPS, THIERRY MAGNAC AND ÉRIC MAURIN}




\title{
Set Identified Linear Models
}

\author{
Christian Bontemps, Thierry Magnac, Eric Maurin ${ }^{\ddagger}$
}

\author{
First version, August 2006 \\ This Version, September 2009
}

\begin{abstract}
We analyze the identification and estimation of parameters $\beta$ satisfying the incomplete linear moment restrictions $E\left(z^{\top}(x \beta-y)\right)=E\left(z^{\top} u(z)\right)$ where $z$ is a set of instruments and $u(z)$ an unknown bounded scalar function. We first provide empirically relevant examples of such a set-up. Second, we show that these conditions set identify $\beta$ where the identified set $B$ is bounded and convex. We provide a sharp characterization of the identified set not only when the number of moment conditions is equal to the number of parameters of interest but also in the case in which the number of conditions is strictly larger than the number of parameters. We derive a necessary and sufficient condition of the validity of supernumerary restrictions which generalizes the familiar Sargan condition. Third, we provide new results on the asymptotics of analog estimates constructed from the identification results. When B is a strictly convex set, we also construct a test of the null hypothesis, $\beta_{0} \in B$, whose size is asymptotically correct and which relies on the minimization of the support function of the set $B-\left\{\beta_{0}\right\}$. Results of some Monte Carlo experiments are presented.
\end{abstract}

\footnotetext{
*Toulouse School of Economics (GREMAQ and IDEI), Toulouse, France, bontemps@ cict.fr ${ }^{\dagger}$ Toulouse School of Economics, (GREMAQ and IDEI), Toulouse, France, magnac@ cict.fr

${ }^{\ddagger}$ Paris School of Economics, Paris, France. Eric.Maurin@ens.fr
} 


\section{Introduction $^{1}$}

Point identification is often achieved by using strong and difficult to motivate restrictions on the parameters of interest. This paper contributes to the growing literature that uses weaker assumptions, under which parameters of interest are set identified only. A parameter is set identified when the identifying restrictions impose that it lies in a set that is smaller than its potential domain of variation, but larger than a single point. We exhibit a class of semi-parametric models in which set identification and estimation can be achieved at low cost and using inference tools close to what is standard in applied work.

In our set-up, parameters of interest are defined by a set of restrictions that we call incomplete linear moment restrictions. Specifically, we consider $y$, a dependent variable, $x$, a vector of $p$ variables and $z$ a vector of $m$ variables and assume that parameter $\beta$ satisfies:

$$
E\left(z^{\top}(x \beta-y)\right)=E\left(z^{\top} u(z)\right)
$$

where $u(z)$ is any single-dimensional measurable function that takes its values in a given bounded interval $I(z)$. One leading example is the familiar linear projection model $y=x \beta+\varepsilon$, where $\varepsilon$ is uncorrelated with $z$, but where the continuous dependent variable, $y$, is censored by intervals. The issue addressed in this paper is to identify and estimate the set, $B$, lying in $\mathbb{R}^{p}$ of all values which satisfy equation (1) for at least one admissible $u($.

A general approach to inference when a set only is identified has recently been proposed by Chernozukov, Hong and Tamer (2007). They define the identified set as the set of zeroes of a functional, called the criterion, and there is no constraint on its shape. In particular, their procedure is valid even when the identified set is neither convex nor bounded. In contrast, the identified set $B$ analysed in this paper is, by construction, bounded and convex. These are key features that we exploit using the concept of a support function which sharply characterize convex sets (Rockafellar, 1970) as Beresteanu and Molinari (2008) did in the linear projection model in which variables $x$ and $z$ are identically the same.

\footnotetext{
${ }^{1}$ This paper was developed for the invited session that one of us gave at ESEM'06 in Vienna. We thank Richard Blundell, Andrew Chesher, Guy Laroque, Whitney Newey, Adam Rosen and particularly Francesca Molinari for helpful discussions as well as three anonymous referees for their insightful comments. We also thank the participants at seminars at PUC-Rio, CEMMAP, CREST Malinvaud seminar, Mannheim, Yale, NYU, Paris I, Cornell, MIT, Northwestern, Toulouse and Cambridge as well as in workshops and conferences (ESRC Bristol '07, Montréal Conference on GMM '07, London Cemmap-Northwestern University Conference on "Inference in Partially Identified Models" '08, Marseille Festschrift for Russell Davidson '08) for comments. We thank Mehtap Akguc for her excellent research assistance. The usual disclaimer applies.
} 
Our first contribution relates to identification. We first show that in the set-up in which there are as many instruments as explanatory variables $(m=p)$, the identified set is necessarily non-empty. The support function of the identified set $B$, whose argument is any direction $q$ of the unit sphere in $\mathbb{R}^{p}$, is sharply characterized as the expectation of a simple random function. In contrast, when there are supernumerary instruments $(m>p)$, the identified set $B$ might be empty. We exhibit a necessary and sufficient condition, a generalization of the usual overidentifying condition à la Sargan, under which the identified set is not empty. It remains bounded and convex and its support function which sharply characterizes it results from the minimization of the expectation of a simple random function. We also exhibit conditions under which the existence of supernumerary instruments restores point identification.

The next contribution of the paper is to provide a simple estimator of the support function of the identified set. This estimator is the empirical analogue of the expectation of the random function to which the support function is equal. In their closely related contribution, Beresteanu and Molinari (2008) provide an estimation procedure for a class of convex identified sets using the theory of random sets. We find it more fruitful to directly use the theory of stochastic process from which the theory of random sets is derived because the results can be obtained under less restrictive conditions and are easier to generalize to the endogenous case. Specifically, when the support function is not differentiable, we show that the estimate converges in distribution at a $\sqrt{n}$ rate to the sum of a Gaussian process and of a process that we characterize and whose support comprises the points of non differentiability only. Given the prevalence of discrete regressors that leads to such non differentiability issues, this generalization is worthy of attention. Interestingly enough, our approach also reveals that the asymptotic results of Beresteanu and Molinari (2008) actually simplify to a quite standard linear model format for the covariance matrix in the case in which the support function is differentiable.

Furthermore and more importantly, we develop a new test procedure for null hypotheses concerning parameter values such as $H_{0}: \beta_{0} \in B$ when the support function is differentiable. We argue that this class of hypotheses is more attractive to economists than hypotheses about sets (such as, say, $H_{0}: B_{0} \subset B$ ). For example, the generalized Sargan condition developed in the supernumerary moment case can be written this way. Moreover, our test has correct asymptotic size and is very easy to adapt to hypotheses about sub-vectors of the complete parameter. The convexity of support functions associated to convex sets is the key feature that simplifies our test 
procedure. The test statistic is constructed as the minimum value of a convex function over the compact unit sphere in a finite-dimensional space. We exploit this characteristic to derive the asymptotic distribution of the test statistic even in the case in which the convex set $B$ has kinks. The form of the test statistic is reminiscent of the test statistic proposed by Galichon \& Henry (2009) in a more general context since the space over which the minimum of the test statistic is taken in our case is much smaller than theirs because of the convexity assumption.

Finally, the same key feature of convexity allows us to derive asymptotic properties of the estimates in the case in which there are supernumerary moment restrictions and the identified set is a proper set. Estimates are uniformly almost surely consistent and when the support function is differentiable, the inflated difference between the estimated and true functions converges to a Gaussian process whose covariance operator can be characterized and estimated simply.

This paper belongs to the growing literature on set identification. From the very start of structural modeling, identification meant point identification. Dispersed in the literature though, there are examples of the weaker concept of set identification. Set identification can come from two broad sets of causes : information might be missing or structural models might not generate enough moment restrictions or inequality restrictions only. The oldest examples of the first case corresponds to measurement errors. They were introduced by Gini (1921), Frish (1934) and further analyzed, decades later, by Klepper and Leamer (1984), Leamer (1987) or Bollinger (1996). There are many other examples of missing information generating incomplete identification (see Manski, 2003 for a survey). Seminal analysis of the incomplete information case include Fréchet (1951), Hoeffding (1940) and Manski (1989) whereas recent applications include Vazquez-Alvarez, Melenberg and van Soest (2001), Blundell, Gosling, Ichimura and Meghir (2007), Honoré and Lleras-Muney (2006) and Ciliberto and Tamer (2009). Horowitz and Manski (1995) consider the case where the data are corrupted or contaminated while Ridder and Moffitt (2007) provide a survey of the results relative to two-sample combination. Structural models delivering moment inequality restrictions (instead of equalities) are the second type of models leading to set identification (Andrews, Berry and Jia, 2002, Pakes, Porter, Ho and Ishii, 2005, Haile and Tamer, 2003, Galichon and Henry, 2009 among others). Set identification can also be generated by discrete exogeneous variation such as in Chesher (2005). In both cases, Chernozhukov, Hong and Tamer (2007) use a criterion approach for the definition of the identified set and subsampling techniques for estimation and inference (see also Romano and Shaikh, 2009). 
In the moment inequality set-up, Rosen (2008) develops simple testing procedures and Bugni (2009) and Canay (2009) investigate the properties of canonical bootstrap and modifications of it. Andrews and Guggenberger (2009) studies cases that do not fall under the assumptions of Imbens and Manski (2004) or Stoye (2009).

The class of models considered in this paper belongs to both branches of the literature. Incomplete linear conditions can be interpreted as a specific set of inequality restrictions generated by some missing information. Yet, our framework is more restrictive than the popular moment inequality restrictions set up since the unknown function $u(z)$ is single dimensional. The leading examples that we propose are derived from partial observation when outcomes are censored by intervals (Manski \& Tamer, 2002, Stoye, 2007, Beresteanu \& Molinari, 2008), when the continuous regressor in a binary model is observed by intervals or is discrete (Magnac \& Maurin, 2008) or when categorical data on opinions and attitudes are analyzed.

Incomplete linear moment conditions define identified sets which are convex and bounded. The approach developed in this paper relies directly on these two properties and we expect that the same procedure can be adapted to other contexts where the identified set is convex and bounded. In contrast, we believe that estimation is more difficult to implement in set-ups such as those proposed by Klepper and Leamer (1984) or Erikson (1993) because the corresponding identified sets are not bounded and convex. Finally, while our results are given in a global linear set-up, their adaptation to a local linear set-up seems to be achievable at low cost.

Section 2 defines the set up of Incomplete Linear Models and develops examples that are of interest for applied econometricians. Section 3 sharply characterizes the identified set. We analyze the case in which the number of parameters is equal to the number of restrictions as well as the case in which the number of restrictions is larger than the number of parameters. In the latter case, we provide the extension of the Sargan condition. For the sake of simplicity, Section 4 specializes to the case of outcomes measured by intervals. Under general conditions, we derive asymptotic properties of estimates in the case of no moment restrictions in surplus. We also develop test procedures, construct confidence regions by inversion of the tests and derive asymptotic properties of the estimates in the case in which there are supernumerary restrictions. Section 5 is devoted to Monte Carlo experiments about estimation and testing procedures and Section 6 concludes. 


\section{The Set-up of Incomplete Linear Models}

In this paper, we analyze the identification and estimation of parameters $\beta$ of what we call an incomplete linear model. In this model, the variables satisfy the incomplete linear moment conditions:

$$
E\left(z^{\top}(x \beta-y)\right)=E\left(z^{\top} u(z)\right)
$$

where $y$ is a scalar dependent variable, $x$ a vector of $p$ covariates, $z$ a vector of $m$ instruments and $u(z)$ a measurable function which takes values ${ }^{2}$ in an admissible set $I(z)=[\underline{\Delta}(z), \bar{\Delta}(z)]$. These two bounds $\underline{\Delta}(z)$ and $\bar{\Delta}(z)$ can be constructed using two observable variables $\bar{y}$ and $\underline{y}$ such that $\bar{y} \geq \underline{y}, y \in[\underline{y} ; \bar{y}]$, and

$$
E(\bar{y}-y \mid z)=\bar{\Delta}(z)>0>E(\underline{y}-y \mid z)=\underline{\Delta}(z) .
$$

The next subsections provide examples, the leading one being that a dependent variable is observed by intervals only so that the lower, $\underline{y}$, and upper bounds, $\bar{y}$, of the interval are explicitly reported in the dataset (e.g. Manski and Tamer, 2002). We assume the following regularity conditions:

\section{Assumption R(egularity):}

R.i. (Dependent variables) $\bar{y}, \underline{y}$ and $y$ are scalar random variables.

R.ii. (Covariates \& Instruments) The support of the distribution $F_{x, z}$ of $(x, z)$ is $S_{x, z} \subset$ $\mathbb{R}^{p} \times \mathbb{R}^{m}$. The dimension of the set $S_{x, z}$ is $r \leq p+m$ where $p+m-r$ are the potential overlaps and functional dependencies. ${ }^{3}$. Furthermore, the conditions of full rank, $\operatorname{rank}\left(E\left(z^{\top} x\right)\right)=p$ and $\operatorname{rank}\left(E\left(z^{\top} z\right)\right)=m$ hold. Finally, $\operatorname{Pr}\{z=0\}=0$.

R.iii. The random vector $(\bar{y}, \underline{y}, x, z)$ belongs to the space $L^{2}$ of square integrable variables.

Along with equation (2), assumptions $R . i-i i$ defines the linear model where there are $p$ explanatory variables and $m$ instrumental variables (assumption R.ii). Assumption R.ii, accommodates the standard exogenous case $x=z$ as a particular case and the absence of a mass point at $\{0\}$ is an assumption simplifying the interpretation later on. Assumption R.iii implies that all cross-moments and regression parameters are well defined. As shown in the next section, it implies that the set of identified parameters is bounded.

\footnotetext{
${ }^{2}$ For ease of notation, all statements referring to any value of a random variable should be understood as this random variable almost surely.

${ }^{3}$ With no loss of generality, the $p$ explanatory variables $x$ can partially overlap with the $m \geq p$ instrumental variables $z$. Variables $(x, z)$ may also be functionally dependent (for instance $x, x^{2}, \log (x), \ldots$ ).
} 


\subsection{Censored Dependent Variables}

The first interesting set of examples corresponds to common linear regression models where the dependent variable $y$ is observed by interval only (see e.g. Manski and Tamer, 2002). Household income, individual wages, hours worked or time spent at school represent continuous outcomes that are often reported by interval in survey or administrative data. ${ }^{4}$ For example, the long standing (and still growing) literature on the long run variations in the distribution of income relies on tax data reporting the number of tax payers for a finite number of income brackets only (see e.g., Piketty, 2005). Researchers typically use parametric extrapolation techniques to estimate the fractiles of the latent income distributions and to analyse variations across periods and countries. The robustness of these analyses to alternative extrapolation assumptions remains unclear, however.

In these examples, the data are given by the distribution of a random vector $w=(\bar{y}, \underline{y}, x)$ where $[y, \bar{y}]$ represents the observed interval ${ }^{5}$ of a latent variable $y^{*}$ and $x$ is a vector of $p$ covariates. The observed bounds $\underline{y}$ and $\bar{y}$ are assumed to satisfy R.iii. ${ }^{6}$ Within this framework, we consider linear latent models :

$$
y^{*}=x \beta+\varepsilon,
$$

where $\varepsilon$ is a random variable uncorrelated with $x, E\left(x^{\top} \varepsilon\right)=0$. The issue is to characterize the set $B$ of parameters $\beta$ such that the latent model defined by equation (4) is consistent with the observed bounds. By definition, $\beta$ belongs to $B$ if and only if there exists a random variable, $\varepsilon$, uncorrelated with $x$ and such that $x \beta+\varepsilon \in[\bar{y}, \underline{y}]$.

Assuming that all variables are in $L^{2}$ so that all cross-moments exist, the following proposition shows that $B$ is defined by an incomplete linear regression of the center of the interval measurement $y=\frac{\bar{y}+\underline{y}}{2}$ on covariates $x$.

Proposition 1 Denote $y=\frac{\bar{y}+\underline{y}}{2}$ the center and $\Delta(x)=E\left(\frac{\bar{y}-\underline{y}}{2} \mid x\right)$ half of the average length of the observed interval $[\underline{y}, \bar{y}]$. Then $\beta$ belongs to $B$ if and only if there exists a measurable

\footnotetext{
${ }^{4}$ Also, for anonymity reasons, only interval information could be made available to researchers even though the information collected is actually continuous.

${ }^{5}$ When the interval is not closed, it is not set $B$ itself but the closure of $B$ which is identified (see Magnac and Maurin, 2008 for more precise statements about closure).

${ }^{6}$ Without this condition, parameter $\beta$ is not identified in the strong sense, i.e. any value of $\beta$ rationalizes the data. It stems from the well known argument that there is no robust estimator for the mean (see Magnac and Maurin, 2007 , for an example).
} 
function $u(x)$ which takes values in $I(x)=[-\Delta(x) ; \Delta(x)]$ such that,

$$
E\left(x^{\top}(x \beta-y)\right)=E\left(x^{\top} u(x)\right) .
$$

Proof. See Appendix A.1

\subsection{Discussion of other Applications}

Other interesting examples correspond to contingent valuation studies where participants are asked whether their willingness-to-pay $\left(w^{*}\right)$ for a good or resource exceeds a bid, $-v$, chosen by experimental design (see e.g., McFadden, 1994). The outcome under consideration $w$ equals one if the respondent willingness-to-pay exceeds the experimental bid (i.e., $w^{*}+v>0$ ) and the relationship of interest between $w^{*}$ and a set of covariates $x$ is to be inferred from available observations on $w, x$ and $v$. Dosage response models are a related example in which $w$ is equal to one when a lethal dose $w^{*}$ exceeds a treatment dose, $-v$, chosen by experimental design. In all these cases, the latent model is written as that $w^{*}=x \beta+\varepsilon$ and the semiparametric binary model $w=1(x \beta+v+\varepsilon>0)$ is estimated under three assumptions. The random term $\varepsilon$ is uncorrelated with regressors $x$ and is independent of regressor $v$ conditional on $x$ (i.e., $F_{\varepsilon}(. \mid x, v)=F_{\varepsilon}(. \mid x)$ ) if only because of experimental design. Also, it is often plausible to suppose that the support of $w^{*}$ is small relative to the support of $v$ (i.e., $\operatorname{Supp}(x \beta+\varepsilon) \subset \operatorname{Supp}(-v)$ ). Assuming that $(x \beta+\varepsilon)$ represents the latent propensity to buy an object and, $-v$, is the price of this object, it simply amounts to assume that for sufficiently high (respectively low) price no one (respectively everyone) buys the object under consideration.

When $v$ is continuously observed and its support is an interval, we are in the case studied by Lewbel (2000) and $\beta$ is point identified. In contrast, when the distribution of $v$ is not continuous, the set $B$ of observationally equivalent parameters is a proper set defined by a moment condition similar to equation (2) (see Magnac and Maurin, 2008).

Categorical data on individual opinions or attitudes provide another potential field of applications. Surveys on job satisfaction or happiness typically contain categorical data on subjective outcomes such as "Taking all things together, how would you say things are these days - would you say you are very happy, fairly happy or not too happy these days?". It is assumed that these responses are function of a continuous intensity measure $y^{*}=x \beta+\varepsilon$ where $\varepsilon$ has a parametric distribution (ordered probit or logit). Alternatively, if the distribution of $\varepsilon$ is unspecified, the identified set of parameters is defined by moment conditions similar to equation (2). 


\section{The Identified Set of Structural Parameters}

This section provides a detailed description of $B$, the set of observationally equivalent parameters, $\beta$, that are compatible with the incomplete linear model above. We first focus on the case in which the number of instruments $z$ is equal to the number of variables $x$ (the exogenous case $z=x$ being a particular example). Second we show how the results can be extended to the case in which the number of instruments $z$ is larger than the number of explanatory variables, $x$.

\subsection{No Moment Conditions in Surplus}

When the number of instruments is equal to the number of variables, the assumption (R.ii) that $E\left(z^{\top} x\right)$ is full rank implies that equation (2) has one and only one solution in $\beta$ for any function $u(z)$ varying in the admissible set. The set of identified parameters, $B$, is the collection of such parameters:

$$
B=\left\{\beta: \beta=\left(E\left(z^{\top} x\right)\right)^{-1} E\left(z^{\top}(y+u(z))\right), u(z) \in[\underline{\Delta}(z), \bar{\Delta}(z)]\right\}
$$

The identified set $B$ is therefore non empty (set e.g. $u(z)=0$ ), convex and closed since the admissible set is convex and closed.

The key object that we exploit is the support function ${ }^{7}$ of a convex set defined as:

$$
\forall q \in \mathbb{R}^{p}, \delta^{*}(q \mid B)=\sup \left\{q^{\top} \beta \mid \beta \in B\right\}
$$

Given that support functions are positively homogenous in $q$, it is sufficient to define them over the unit sphere of $\mathbb{R}^{p}$ i.e. $\mathbb{S}=\left\{q \in \mathbb{R}^{p} ;\|q\|=1\right\}$. Furthermore, following Rockafellar (1970), set $B$ can be unambiguously characterized as:

$$
\beta \in B \Leftrightarrow \forall q \in \mathbb{S}^{p}, q^{\top} \beta \leq \delta^{*}(q \mid B)
$$

and identification of $B$ is therefore equivalent to the identification of its support function $\delta^{*}(. \mid$ $B)$.

We now show that the support function of $B$ can be written as a population moment of two simple random variables. Let $\beta$ a point which belongs to set $B$. From (6), there exists some function $u(z) \in[\underline{\Delta}(z), \bar{\Delta}(z)]$ such that

$$
\beta=\left(E\left(z^{\top} x\right)\right)^{-1} E\left(z^{\top}(y+u(z))\right) .
$$

\footnotetext{
${ }^{7}$ Beresteanu and Molinari (2008) also use this function in order to apply the theory of random set variables.
} 
We can multiply this equation by vector $q$ to express:

$$
q^{\top} \beta=q^{\top}\left(E\left(z^{\top} x\right)\right)^{-1} E\left(z^{\top}(y+u(z))\right)=E\left(z_{q}(y+u(z))\right)
$$

where $z_{q}=q^{\top} \Sigma^{\top} z^{\top}$ and $\Sigma=\left(E\left(x^{\top} z\right)\right)^{-1}$. As the support function in the direction $q$ is the supremum of $q^{\top} \beta$ when $\beta \in B$, it is the supremum of (7) over the set of admissible $u(z) \in$ $[\underline{\Delta}(z), \bar{\Delta}(z)]$. It reduces to a simple single-dimensional optimization problem whose solution is given by:

Proposition 2 Let $w_{q}=\underline{y}+1\left\{z_{q}>0\right\}(\bar{y}-\underline{y})$. The support function of $B$ is equal to:

$$
\delta^{*}(q \mid B)=E\left(z_{q} w_{q}\right)
$$

The interior of $B$ is not empty and $\beta_{q}=\left(E\left(z^{\top} x\right)\right)^{-1} E\left(z^{\top} w_{q}\right)$ is a frontier point of $B$ such that $\delta^{*}(q \mid B)=q^{\top} \beta_{q}$.

\section{Proof. See Appendix B.1}

This proposition sharply characterizes set $B$. The support function is well defined because assumption (R.iii) ensures that all cross-moments are well defined. In particular, the support function is bounded and therefore set $B$ is bounded. Furthermore, as a convex function, $\delta^{*}(q \mid B)$ is differentiable except at a countable number of points in a set $D_{f}$. The following lemma provides geometric properties of set $B$ and an explicit characterisation of $D_{f}$.

Lemma 3 The support function $\delta^{*}(q \mid B)$ is differentiable on $\mathbb{S}$ except on a set $D_{f}$ which is composed of directions $q \in \mathbb{S}$ such that $\operatorname{Pr}\left(z_{q}=0\right)$ is positive. The directions in $D_{f}$ are orthogonal to exposed faces of the identified set.

\section{Proof. See Appendix B.2}

Exposed faces of the identification set $B$ are intersections of $B$ and supporting hyperplanes that are not reduced to singletons (see Rockafellar, 1970, pp.162-163) and set $D_{f}$ is not empty for instance when some variables $z$ have mass points. Defining this set turns out to be important for asymptotic properties derived in the next section. One Monte Carlo experiment in Section 5.3 analyzes the common case in which one explanatory variable is a dummy variable. 


\subsection{Supernumerary Moment Conditions}

We consider now that the dimension, $m$, of the random vector $z$ is larger than the dimension, $p$, of covariates $x$ and we denote $x(z)$ the linear projection of $x$ onto instruments $z$, i.e., $x(z)=z E\left(z^{\top} z\right)^{-1} E\left(z^{\top} x\right)$. Without loss of generality, we assume that the $m-p$ supernumerary instruments $z^{s}=\left(z_{p+1}, \ldots, z_{m}\right)$ provide supernumerary moment conditions in the sense that no linear combination of these additional instruments is linearly dependent of $x(z)$. These instruments always exist because of the rank condition R.iii. Formally, if $\zeta^{s}$ denotes the vector of residuals of the linear projection of these $m-p$ instruments onto $x(z)$, we assume rank $\left(E\left(\zeta^{s^{\top}} \zeta^{s}\right)\right)=m-p$. It may very well be the case that other subsets of $m-p$ instruments satisfy this condition, but, as discussed in Appendix B.3, our results do not depend on the choice of a specific subset.

The parameters of interest $\beta$ satisfy the incomplete linear moment conditions (2):

$$
E\left(z^{\top} x\right) \beta=E\left(z^{\top}(y+u(z))\right)
$$

and the identified set $B$ is again closed, convex and bounded. The first two properties hold as before because the moment conditions are linear and the admissible set $I(z)$ containing $u(z)$ is closed and convex. To show that $B$ is bounded, we can always restrict equation (8) to a subset of $p$ instruments, say $x(z)$, and construct the identified region as in the previous section. The true identified set is included in this identified region.

Yet in contrast to the case in which $(m=p)$, the identified set $B$ could be empty. In the next sub-section, we derive a necessary and sufficient condition which generalizes the usual over-identifying condition à la Sargan. To do that, we need new notations. Define first $z_{F}$ the ortho-normalization of the linear projection $x(z)$ defined above

$$
z_{F}=x(z) E\left(x(z)^{\top} x(z)\right)^{-1 / 2}
$$

Define also $z_{H}$ the ortho-normalization of the residuals $\zeta^{s}$ of the projection of the supernumerary instruments $z^{s}$ onto $z_{F}$. Analytically, $z_{H}=\zeta^{s} E\left(\zeta^{s \top} \zeta^{s}\right)^{-1 / 2}$ where,

$$
\zeta^{s}=z^{s}-z_{F} E\left(z_{F}^{\top} z^{s}\right)=z^{s}-x(z) E\left(x(z)^{\top} x(z)\right)^{-1} E\left(x(z)^{\top} z^{s}\right) .
$$

After some algebraic manipulations, we have $E\left(\zeta^{s \top} x\right)=0_{m-p, p}$ and, consequently, $E\left(z_{H}^{\top} x\right)=$ $0_{m-p, p}$. 


\subsubsection{The Validity of Supernumerary Moment Conditions}

Both vectors $z_{F}$, of dimension $p$, and $z_{H}$, of dimension $m-p$, are linear combinations of the $m$ instruments $z$, so that equation (8) implies,

$$
E\left(z_{F}^{\top} x\right) \beta=E\left(\left(z_{F}^{\top}(y+u(z))\right) \text { and } E\left(z_{H}^{\top} x\right) \beta=E\left(z_{H}^{\top}(y+u(z))\right)\right.
$$

As $E\left(z_{H}^{\top} x\right)=0_{m-p, p}$, the second set writes $E\left(z_{H}^{\top}(y+u(z))\right)=0$. Not only these two sets of restrictions are necessary, but they can be proven to be sufficient:

Lemma 4 Parameter $\beta$ belongs to $B$ if and only if there exists $u(z)$ in $[\underline{\Delta}(z), \bar{\Delta}(z)]$ such that:

$$
\begin{aligned}
& E\left(z_{F}^{\top} x\right) \beta=E\left(z_{F}^{\top}(y+u(z))\right) \\
& E\left(z_{H}^{\top}(y+u(z))\right)=0
\end{aligned}
$$

Proof. See Appendix B.3

Interestingly enough, the second set of restrictions does not depend on $\beta$ whereas the first set provides a one-to-one relationship between admissible $u(z)$ and admissible $\beta$. It follows that $B$ is non empty if and only if there is $u(z)$ in $[\underline{\Delta}(z), \bar{\Delta}(z)]$ such that

$$
E\left(z_{H}^{\top}(y+u(z))\right)=0
$$

Denote $B_{\text {Sargan }}$ the identified set of parameters of the incomplete regression of $y$ on the supernumerary instruments $z_{H}$, i.e.:

$$
\begin{aligned}
B_{\text {Sargan }} & =\left\{\gamma: E\left(z_{H}^{\top}\left(z_{H} \gamma-y\right)\right)=E\left(z_{H}^{\top} u(z)\right), u(z) \in[\underline{\Delta}(z), \bar{\Delta}(z)]\right\} \\
& =\left\{\gamma: \gamma=E\left(z_{H}^{\top}(y+u(z))\right), u(z) \in[\underline{\Delta}(z), \bar{\Delta}(z)]\right\} \subset \mathbb{R}^{m-p} .
\end{aligned}
$$

The adapted Sargan condition given by equation (11) means that $B_{\text {Sargan }}$ contains the point $\gamma=0$, that is $O_{m-p}$, the origin point of $\mathbb{R}^{m-p}{ }^{8}$

Proposition 5 The two following conditions are equivalent:

i. B is not empty,

ii. $B_{\text {Sargan }} \ni O_{m-p}$.

\footnotetext{
${ }^{8}$ As discussed at the end of the proof of Lemma 4, the adapted Sargan condition imposes restrictions on the set of admissible $u(z)$ which do not depend on the choice of supernumerary instruments, $z_{H}$.
} 
Proof. Using the previous developments.

This extends the usual overidentification restrictions. When moment conditions are complete, the set of admissible $u(z)$ is reduced to $\{0\}$ and the set $B_{\text {Sargan }}$ is reduced to the point $E\left(z_{H}^{\top} y\right)$. The Sargan or J-test consists in testing $O_{m-p} \in B_{\text {Sargan }}=\left\{E\left(z_{H}^{\top} y\right)\right\}$ or equivalently that $E\left(z_{H}^{\top} y\right)=0$. In section 4, we will construct a general test for the assumption $H_{0}: \beta_{0} \in B$, when $B$ is the identified region of an incomplete linear moment model. It will provide us with a direct way for testing the Sargan condition given in Proposition 5. Before, the next subsection provides a characterization of the identified set when there are supernumerary moment conditions.

\subsubsection{Geometric and Analytic Characterization of the Identified Set}

Assuming that the Sargan condition holds true, the identified set $B$ is defined by the incomplete moment conditions:

$$
E\left(z^{\top}(x \beta-y)\right)=E\left(z^{\top} u(z)\right) \text { subject to } u(z) \in[\underline{\Delta}(z), \bar{\Delta}(z)] \text {. }
$$

This set of restrictions can be rewritten by introducing auxiliary parameters, $\gamma$, as:

$$
\left\{\begin{array}{l}
E\left(z^{\top}\left(x \beta+z_{H} \gamma-y\right)\right)=E\left(z^{\top} u(z)\right) \\
\gamma=0
\end{array}\right.
$$

under the same constraint for $u(z)$. Let $B_{U}$ ( $U$ for unconstrained) be the set of $m$ parameters $(\beta, \gamma)$ satisfying the relaxed program,

$$
E\left(z^{\top}\left(x \beta+z_{H} \gamma-y\right)\right)=E\left(z^{\top} u(z)\right), \text { subject to } u(z) \in[\underline{\Delta}(z), \bar{\Delta}(z)]
$$

An interesting feature of this relaxed program is that the number of explanatory variables $m$ is equal to the number of moment conditions, and no moments are in surplus. Consequently, the support function of $B_{U}$ can be characterized using Proposition 2. The second interesting feature of this construction is that the identified set $B$ is equal to the intersection of $B_{U}$ and the hyperplane defined by $\gamma=0$. General results for the support function of intersection of convex sets (Rockafellar, 1970) can be used to characterize set $B$ and this yields:

Proposition 6 Let $q$ a vector of $\mathbb{R}^{p}$ and $(q, \lambda)$ a vector of $\mathbb{R}^{m}$. We have:

$$
\delta^{*}(q \mid B)=\inf _{\lambda} \delta^{*}\left((q, \lambda) \mid B_{U}\right) .
$$


Proof. Rockafellar (1970) and Appendix B.4

The geometric intuition is the following. For any point $\beta_{f} \in \partial B$, the frontier of $B$, there always exists one projection direction such that the projection of $B_{U}$ onto $\gamma=0$ into this direction, admits $\beta_{f}$ as a frontier point. The vector $\lambda_{m}(q)$ characterizes this projection direction. It corresponds to a tangent space (not necessarily unique) of $B_{U}$ at $\beta_{f}$.

Note also that the orthogonal projection of $B_{U}$ onto $\gamma=0$,

$$
\left\{\beta \in \mathbb{R}^{p}, \exists u(z) \in[\underline{\Delta}(z), \bar{\Delta}(z)], \beta=\left(E\left(z_{F}^{\top} x\right)\right)^{-1} E\left(z_{F}^{\top}(y+u(z))\right)\right\},
$$

is equal to the set of unconstrained solutions to equation (9). This projection contains set $B$ since parameters in set $B$ are generated by functions $u(z)$ satisfying also condition (10). Supernumerary restrictions therefore reduce the size of the identified set and generically they strictly do so. ${ }^{9}$

\subsubsection{Supernumerary Moment Conditions as a Way to Restore Point Identification}

The adapted Sargan condition $\left(O \in B_{\text {Sargan }}\right)$ imposes restrictions on the size of the set of admissible functions $u(z)$ and, consequently, on the size of the identified set $B$. This section explores whether $B$ can eventually be reduced to a singleton and point identification be restored.

When the point $O_{m-p}$ belongs to the interior of $B_{\text {Sargan }}$, functions which satisfy the Sargan condition (11) are not unique and set $B$ has necessarily a non empty interior. More interesting cases arise when $O_{m-p}$ belongs to the frontier of $B_{\text {Sargan }}$. Using the proof of Proposition 2, the frontier points of $B_{\text {Sargan }}$ are generated by functions $u_{q}^{\text {Sargan }}(z)$ defined as,

$$
u_{q}^{\text {Sargan }}(z)=(\bar{\Delta}(z)-\underline{\Delta}(z)) \mathbf{1}\left\{z_{q}>0\right\}+\Delta^{*}(z) \mathbf{1}\left\{z_{q}=0\right\}
$$

where $\Delta^{*}(z)$ can be any function taking values in $[\underline{\Delta}(z), \bar{\Delta}(z)]$.

Suppose first that $O_{m-p}$ lies on an exposed face of $B_{\text {Sargan }}$ and let $q_{O}$ the vector of $\mathbb{S}_{m-p}$ orthogonal to this face. As Lemma 3 implies that $\operatorname{Pr}\left\{z_{H q_{O}}=0\right\}>0$, the generating function $u_{q_{O}}^{\text {Sargan }}(z)$ is not unique and the identified set is not reduced to a singleton. In contrast, if $O_{m-p}$ is not on an exposed face of $B_{\text {Sargan }}, u_{q_{O}}^{\text {Sargan }}(z)$ is unique and the set $B$ is reduced to a singleton $\left\{\beta_{0}\right\}$ defined as:

$$
\beta_{0}=\left(E\left(z_{F}^{\top} x\right)\right)^{-1} E\left(z_{F}^{\top}\left(y+u_{q_{O}}^{\text {Sargan }}(z)\right)\right) .
$$

We summarize this result in:

\footnotetext{
${ }^{9}$ Appendix B.5 shows that the resulting set $B$ does not depend on which version of $z_{H}$ was chosen and thus on which version of unconstrained $B_{U}$ was selected.
} 
Proposition 7 If $O_{m-p}$ belongs to the frontier $\partial B_{\text {Sargan }}$ albeit not to an exposed face of $B_{\text {Sargan }}$, parameter $\beta$ is point-identified.

\section{Estimation and Inference}

This section provides a description of how we estimate the support function of $B$ and how we test hypotheses of interest. We will deal only with random samples $i=1, ., n$, where $\left(\bar{y}_{i}, \underline{y}_{i}, y_{i}, x_{i}, z_{i}\right)$ is observed in the data and independently and identically distributed. ${ }^{10}$ We start by analysing the case where there is no supernumerary moment conditions.

\subsection{Asymptotic Properties: No Supernumerary Moment Conditions}

In this section, we provide an estimate of the support function of the identified set $B$ as characterized in Proposition 2 :

$$
\delta^{*}(q \mid B)=E\left(z_{q} w_{q}\right)
$$

To apply the analogy principle, we first construct $\hat{\Sigma}_{n}$ a bounded estimate ${ }^{11}$ of $\left(E\left(x^{\top} z\right)\right)^{-1}$ and we define for any $i$ :

$$
\begin{aligned}
z_{n, q i} & =z_{i} \hat{\Sigma}_{n} q \\
w_{n, q i} & =\mathbf{1}\left\{z_{n, q i}>0\right\}\left(\bar{y}_{i}-\underline{y}_{i}\right)+\underline{y}_{i} .
\end{aligned}
$$

We define the estimate of $\delta^{*}(q \mid B)$ as:

$$
\hat{\delta}_{n}^{*}(q \mid B)=\frac{1}{n} \sum_{i=1}^{n} z_{n, q i} w_{n, q i}=q^{\top} \hat{\Sigma}_{n}^{\top}\left(\frac{1}{n} \sum_{i=1}^{n} z_{i}^{\top} w_{n, q i}\right) .
$$

Under usual conditions (White, 1999, p35), the estimate $\hat{\delta}_{n}^{*}(q \mid B)$ is uniformly consistent.

Proposition 8 Assume that there exist $M>0$ and $\gamma>0$, such that $\|\Sigma\|^{1+\gamma}, E\left(\left\|x^{\top} z\right\|^{1+\gamma}\right)$, $E\left(\left\|z^{\top} \bar{y}\right\|^{1+\gamma}\right)$ and $E\left(\left\|z^{\top} \underline{y}\right\|^{1+\gamma}\right)$ are bounded by $M$. Then, $\hat{\delta}_{n}^{*}(q \mid B)$ is, uniformly over $\mathbb{S}$, strongly consistent:

$$
\hat{\delta}_{n}^{*}(q \mid B) \stackrel{\text { a.s.u. }}{\rightarrow} \delta^{*}(q \mid B) .
$$

\footnotetext{
${ }^{10}$ Note that it precludes pre-estimation of the bounds as in Magnac and Maurin (2008). We leave this extension for future work.

${ }^{11}$ See Appendix $\mathrm{C}$ for the exact definition where the usual estimate is trimmed to make it bounded.
} 
Proof. See Beresteanu and Molinari, 2008 and Additional Appendix E

The proof builds on the fact that the expression $z_{q} w_{q}$ within the expectation defining $\delta^{*}(q \mid B)$ can be written as a random function $f_{(q, \Sigma)}\left(z_{i}, \bar{y}_{i}, \underline{y}_{i}\right)$ indexed by parameter $(q, \Sigma) \in \Theta=\mathbb{S} \times$ $\{\|\Sigma\| \leq M\}$. Under the conditions of Proposition 8, the parametric class of functions $f_{(q, \Sigma)}$ is Glivenko-Cantelli. If $\Sigma$ is known, the empirical expectation of $f_{(q, \Sigma)}$ converges almost surely to $\delta^{*}(q \mid B)$ uniformly over $\Theta$ under the conditions stated above. Using results for parametric classes (van der Vaart, 1998), we can replace $\Sigma$ by a bounded consistent estimate $\hat{\Sigma}_{n} \in\{\|\Sigma\| \leq M\}$ and the same result holds true.

We use similar reasoning to derive the asymptotic distribution of the estimate by considering the stochastic process defined on $\mathbb{S}$ :

$$
\tau_{n}(q)=\sqrt{n}\left(\hat{\delta}_{n}^{*}(q \mid B)-\delta^{*}(q \mid B)\right)=\sqrt{n}\left(\frac{1}{n} \sum z_{n, q i} w_{n, q i}-E\left(z_{q} w_{q}\right)\right),
$$

whose asymptotic behavior is characterized under usual conditions (White, 1999, p. 118) in the following.

Proposition 9 Assume that there exist $M>0$ and $\gamma>0$ such that $\|\Sigma\|^{2+\gamma}, E\left(\left\|x^{\top} z\right\|^{2+\gamma}\right)$, $E\left(\left\|z^{\top} \bar{y}\right\|^{2+\gamma}\right)$ and $E\left(\left\|z^{\top} \underline{y}\right\|^{2+\gamma}\right)$ are bounded by $M$. The process, $\tau_{n}(q)$ uniformly converges in distribution when $n$ tends to $\infty$ to the sum of a Gaussian stochastic process centered at zero and of a point process which is asymptotically equivalent to :

$$
E_{z}\left(\left|\eta^{\top} W^{1 / 2}\left(I_{p} \otimes q\right) z_{i}^{\top}\right|\left(\bar{y}_{i}-\underline{y}_{i}\right)\left(\mathbf{1}\left\{z_{i} \Sigma q=0\right\}\right)\right) / 2
$$

where $W$ is the asymptotic variance of vec $\left(\hat{\Sigma}_{n}^{\top}\right)$ and whereas $\eta$ is a normally distributed random vector of dimension $p^{2}$, independent of $(\bar{y}, \underline{y}, z)$. Vectors $q$ at which this process are non zero are orthogonal to the exposed faces of B (see Lemma 3).

When set $B$ has no exposed faces, $\tau_{n}(q)$ uniformly converges in distribution when $n$ tends to $\infty$ to a Gaussian stochastic process centered at zero. The covariance function of this process for vectors $(q, r) \in \mathbb{S}$ is,

$$
E\left(z_{q i} \varepsilon_{q i} \varepsilon_{r i} z_{r i}\right)
$$

where $\varepsilon_{q}=w_{q}-x\left(E\left(z^{\top} x\right)\right)^{-1} E\left(z^{\top} w_{q}\right)$ are the residuals of the IV regression of $w_{q}$ on $x$ using instruments $z$.

Proof. See Appendix C.1 
When there are no exposed faces, Beresteanu and Molinari (2008) already derived the asymptotic distribution of $\tau_{n}(q)$ using the formalism of set valued random variables. Proposition 9 provides an alternative characterization of the covariance function of the Gaussian process in terms of residuals $\varepsilon_{q}$ that are easy to compute. When there are exposed faces, this Proposition provides the original result that the limit in distribution is the sum of a Gaussian process and a countable point process which takes non zero values at directions $q$ orthogonal to exposed faces of set $B$.

\subsection{Tests}

This section provides testing procedures for null hypothesis such as $H_{0}: \beta_{0} \in B$, where $\beta_{0}$ represents a potential parameter value. The proposed tests have the correct asymptotic size. Similar approaches could be used for testing assumption about sets, such as $H_{0}: B_{0}=B$, where $B_{0}$ represents a potential value of the identified set. We will focus on the case where the identified set $B$ has no exposed faces (see Lemma 3 for deep conditions on the support of $z$ ) so that the estimate of the support function is asymptotically Gaussian (see Proposition 9). ${ }^{12}$

\section{Assumption D: The support function $\delta^{*}(q \mid B)$ is differentiable everywhere.}

Assumption D does not exclude cases where the derivative of the support function has the same value at different points of the unit sphere. Put differently, it does not exclude that some points of the surface of $B$ may have several different tangent spaces (kinks). In such a case, the relationship between directions of the unit sphere $q$ and points of the surface of $B$ is not one-to-one anymore, which complicates the testing procedure. Set-ups where $B$ has kinks are characterized at the end of the proof of Lemma 3, one leading example being when the density function of some instruments is not positive over the whole real line.

Within this framework, an alternative characterization of $H_{0}: \beta_{0} \in B$ is:

$$
\begin{aligned}
\beta_{0} \in B & \Longleftrightarrow \forall q \in \mathbb{S}, T_{\infty}\left(q ; \beta_{0}\right)=\delta^{*}(q \mid B)-q^{\top} \beta_{0} \geq 0 \\
& \Longleftrightarrow \min _{q \in \mathbb{S}} T_{\infty}\left(q ; \beta_{0}\right) \geq 0,
\end{aligned}
$$

as $\mathbb{S}$ is compact. If we knew a minimizer $q_{0}$ of $T_{\infty}\left(q ; \beta_{0}\right)$, we could consider the empirical analog

\footnotetext{
${ }^{12}$ The difficulty with dealing with the additional point process is that the limit is not asymptotically equicontinuous. We leave this subject for future research.
} 
of $T_{\infty}\left(q_{0} ; \beta_{0}\right)$ :

$$
T_{n}\left(q_{0} ; \beta_{0}\right)=\hat{\delta}_{n}^{*}\left(q_{0} \mid B\right)-q_{0}^{\top} \beta_{0},
$$

and use that $\sqrt{n}\left(T_{n}\left(q_{0} ; \beta_{0}\right)-T_{\infty}\left(q_{0} ; \beta_{0}\right)\right)$ is asymptotically normally distributed and have variance $V_{q_{0}}=V\left(z_{q_{0}}^{\top} \varepsilon_{q_{0}}\right)$. Observe that, when the point $\beta_{0}$ belongs to the frontier of the set, $T_{\infty}\left(q_{0} ; \beta_{0}\right)=0$ and $\sqrt{n} T_{n}\left(q_{0} ; \beta_{0}\right) / \sqrt{V_{q_{0}}}$ qualifies as a test statistics for $H_{0}$.

The two issues that we have to deal with are (i) $q_{0}$ is not known (ii) it needs not be unique if set $B$ has kinks. We thus have to select one admissible $q_{0}$ and replace it by an estimate. The next Proposition shows how to address the second issue by perturbing function $T_{\infty}\left(q ; \beta_{0}\right)$ and the first issue by minimizing the empirical analogue of such a function.

Proposition 10 Under Assumption D and conditions given in Proposition 9, there exist two sequences $v_{0, n} \in \mathbb{S}$ and $a_{n} \in \mathbb{R}^{+}$characterized in the proof, such that any sequence $q_{n}$ of local minimizers of the perturbed program :

$$
\hat{\Psi}_{n, a_{n}}\left(q ; \beta_{0}\right)=T_{n}\left(q ; \beta_{0}\right)-a_{n} q^{\top} v_{0, n},
$$

converges, when $n$ tends to $\infty$, to a single minimizer $q_{0}^{*}$ of $T_{\infty}\left(q ; \beta_{0}\right)$. Then,

$$
\left\{\begin{array}{cc}
\sqrt{n} T_{n}\left(q_{n} ; \beta_{0}\right) / \sqrt{\hat{V}_{n}} \underset{n \rightarrow \infty}{\stackrel{d}{\longrightarrow}} \mathcal{N}(0,1), \quad \text { if } \beta_{0} \in \partial B, \\
\sqrt{n} T_{n}\left(q_{n} ; \beta_{0}\right) / \sqrt{\hat{V}_{n}} \underset{n \rightarrow \infty}{\stackrel{a . s .}{\longrightarrow}}+\infty, \quad \text { if } \beta_{0} \in \operatorname{int}(B), \\
\sqrt{n} T_{n}\left(q_{n} ; \beta_{0}\right) / \sqrt{\hat{V}_{n}} \underset{n \rightarrow \infty}{\stackrel{a . s}{\longrightarrow}}-\infty, \quad \text { if } \beta_{0} \notin B,
\end{array}\right.
$$

where $\hat{V}_{n}=\widehat{V}\left(z_{n, q_{n}} \varepsilon_{n, q_{n}}\right)$ is a consistent estimator of $V_{q_{0}^{*}}$.

Proof. See appendix C.2

Two special cases in which there is no need to actually perturb the program and $a_{n}$ can be set to zero are worth noticing. First, when set $B$ has no kink, for instance when the density of $z$ is positive everywhere, $T_{\infty}\left(q ; \beta_{0}\right)$ is strictly convex and any sequence of local minimizers of $T_{n}\left(q ; \beta_{0}\right)$ tends to the unique minimizer of $T_{\infty}\left(q ; \beta_{0}\right)$. Second, we can set $a_{n}$ to zero when we test a single component of $\beta_{0}$, or a single linear combination of components since there is no kink in the single dimensional case.

Critical regions with asymptotical level $\alpha$ for two interesting null hypotheses can be constructed:

- Test 1: $H_{0}: \beta_{0} \in B$ against $H_{a}: \beta_{0} \notin B$. The critical region $W_{n}^{1}(\alpha)$ is defined by:

$$
W_{n}^{1}(\alpha)=\left\{\beta_{0} \in \mathbb{R}^{p}, \sqrt{n} T_{n}\left(q_{n} ; \beta_{0}\right) / \sqrt{\hat{V}_{n}}<\mathcal{N}_{\alpha}\right\}
$$


- Test 2: $H_{0}: \beta_{0} \in \partial B$ against $H_{a}: \beta_{0} \notin \partial B$. The critical region $W_{n}^{2}(\alpha)$ is:

$$
\left.W_{n}^{2}(\alpha)=\left\{\beta_{0} \in \mathbb{R}^{p}, \mid \sqrt{n} T_{n}\left(q_{n} ; \beta_{0}\right) / \sqrt{\hat{V}_{n}}\right) \mid>\mathcal{N}_{1-\frac{\alpha}{2}}\right\}
$$

where $\mathcal{N}_{\alpha}$ denotes the $\alpha$-quantile of the standard normal distribution and where $q_{n}$ is defined by Proposition 10. In addition, the test statistics is asymptotically pivotal so that we could enhance its finite sample properties by bootstrapping it.

We are specifically interested by the first test. The second one is also of practical interest for instance when testing whether supernumerary instruments help in recovering point identification (i.e., for testing $\left.O \in \partial B_{\text {Sargan }}\right)$.

\subsection{Confidence Regions}

By inverting the first test developed previously with a level of significance equal to $\alpha$, we can construct confidence regions of nominal size asymptotically equal to $100-100 \alpha \%$. Following Lehmann \& Romano (2005), the confidence region $C I_{\alpha}^{n}$ is the collection of parameters $\beta \in \mathbb{R}^{d}$ for which the null hypothesis is not rejected i.e. which does not belong to $W_{n}^{1}(\alpha)$. The following proposition expresses this statement and Appendix E.2 provides a simple way of constructing the confidence region.

Proposition 11 Let $\alpha$ be a significance level, and let $C I_{\alpha}^{n}$ be the set of points of $\mathbb{R}^{p}$ such that $\xi_{n}(\beta)>\mathcal{N}_{\alpha}$, where

$$
\xi_{n}(\beta)=\frac{\sqrt{n} T_{n}\left(q_{n} ; \beta\right)}{\sqrt{\hat{V}_{n}}},
$$

where $\hat{V}_{n}$ is defined in Proposition 10. Under the conditions of Proposition 10,

$$
\lim _{n \rightarrow+\infty} \inf _{\beta \in B} \operatorname{Pr}\left(\beta \in C I_{\alpha}^{n}\right)=1-\alpha .
$$

The limit expressed in the proposition is valid for a fixed data generating process leading to the identification of a proper set $B$. It is not uniformly valid for all data generating processes even if they satisfy the condition, under which we work, that the corresponding identified set B has a non-empty interior. As a consequence, the confidence region is not uniformly asymptotically of nominal size equal to $(1-\alpha)$. However uniformity is important as we might never know, in practice, how far we are from a just identified case.

For simplicity, assume for the remaining part of the section that set $B$ is strictly convex and smooth i.e. the support function is differentiable and strictly convex. Let us consider the limit 
case in which set $B=\left\{\beta_{0}\right\}$. If we construct a confidence region for the parameters using the last Proposition the coverage probability will tend to $1-2 \alpha$ (see Additional Appendix E.3). Indeed, the statistics developed above is discontinuous with respect to the diameter of the identified set at the boundary i.e. when the diameter is equal to zero. ${ }^{13}$

The construction of Imbens and Manski (2004) is uniformly valid in a context of single dimensional sets and more recently, Stoye (2009) clarified the conditions under which this result can be obtained. We can adapt Lemma 4 in Imbens and Manski (2004) to our set-up where the length of the interval is replaced by the diameter of the set and construct a uniform confidence region (see Additional Appendix E.4) ${ }^{14}$. A consequence of what was shown in appendix B.1, is that the diameter of $B$ is positive.

\subsection{Asymptotic Properties: The Supernumerary Case}

We use the characterization given in equation (13) in Proposition 6. If $q$ is a vector of $\mathbb{R}^{p}$ and $(q, \lambda)$ a vector of $\mathbb{R}^{m}$, we have:

$$
\left.\delta^{*}(q \mid B)=\inf _{\lambda} \delta^{*}\left((q, \lambda) \mid B_{U}\right)\right)
$$

On top of assumption D, we assume that:

Condition S: The infimum is attained at values, $\lambda_{m}(q)$, which belong to a compact set $\Lambda \subset R^{m-p}$

The ultimate part of the proof of Proposition 6 exhibits the necessary and sufficient condition under which this is obtained and which, combined with assumption D of differentiability, implies that set $B$ is a proper set.

Let $\hat{\delta}_{n}^{*}\left((q, \lambda) \mid B_{U}\right)$ the estimate of $\delta^{*}\left((q, \lambda) \mid B_{U}\right)$ as derived in Section 4.1 and such that, by Proposition 8:

$$
\hat{\delta}_{n}^{*}\left((q, \lambda) \mid B_{U}\right) \stackrel{\text { a.s.u. }}{\rightarrow} \delta^{*}\left((q, \lambda) \mid B_{U}\right),
$$

and, by Proposition 9, under assumption D:

$$
\tau_{n}^{U}((q, \lambda))=\sqrt{n}\left(\hat{\delta}_{n}^{*}\left((q, \lambda) \mid B_{U}\right)-\delta^{*}\left((q, \lambda) \mid B_{U}\right)\right)
$$

\footnotetext{
${ }^{13}$ The diameter of the set $B$ is the maximum of $\delta(q \mid B)+\delta(-q \mid B)$ on the compact unit sphere.

${ }^{14}$ Stoye also extends the construction of the confidence region to the case in which the estimated size is not a superefficient estimator of the true one although it remains asymptotically normal. In a more general case, Andrews and Guggenberger (2009) focus on the construction of confidence regions using subsampling techniques when the assumption of asymptotic normality is no longer valid.
} 
uniformly converges to a Gaussian process when $n$ tends to infinity.

For any $q$, define:

$$
\hat{\lambda}_{n}(q) \in \arg \min _{\lambda \in \Lambda}\left[\hat{\delta}_{n}^{*}\left((q, \lambda) \mid B_{U}\right)+a_{n} \lambda^{\top} \lambda\right]
$$

where $a_{n}$ is a sequence converging to zero with $n$, defined in the proof below. The estimate $\hat{\lambda}_{n}(q)$ is a solution to a perturbed objective function as in Section 4.2. Define the estimate of the support function of the identified set as:

$$
\hat{\delta}_{n}^{*}(q \mid B)=\hat{\delta}_{n}^{*}\left(\left(q, \hat{\lambda}_{n}(q)\right) \mid B_{U}\right)
$$

The same kind of proof as in Sections 4.1 and 4.2 then applies. Estimating $\hat{\lambda}_{n}(q)$ does not affect the consistency and asymptotic normality of the support function estimates.

Proposition 12 Under the conditions stated in Proposition 9 and conditions $D$ and S, we have:

$$
\hat{\delta}_{n}^{*}(q \mid B) \stackrel{\text { a.s.u. }}{\rightarrow} \delta^{*}(q \mid B)
$$

and:

$$
\tau_{n}(q)=\sqrt{n}\left(\hat{\delta}_{n}^{*}(q \mid B)-\delta^{*}(q \mid B)\right)
$$

converges uniformly to a Gaussian process when $n$ tends to infinity. The Gaussian process has expectation equal to zero and its covariance operator for two directions $(q, r) \in \mathbb{S}$ is given by:

$$
E\left(z_{\left(q, \hat{\lambda}_{n}(q)\right)} \varepsilon_{\left(q, \hat{\lambda}_{n}(q)\right)} \varepsilon_{\left(r, \hat{\lambda}_{n}(r)\right)} z_{\left(r, \hat{\lambda}_{n}(r)\right)}\right)
$$

Proof. See appendix C.3

\section{Monte-Carlo Experiments}

In this section, we develop three simple experiments to assess the performance of our inference and test procedures. In these experiments, the dependent variable is bounded and censored by intervals and the identified set is of dimension 2 for simplicity. In the first two experiments, the frontier of the identified set has no kinks and no exposed faces. In the first experiment, the number of instruments is the same as the number of parameters while we use one supernumerary instrument in the second experiment. We explore the case of an identified set that is neither smooth nor strictly convex in the third experiment. 


\subsection{Smooth and Strictly Convex Sets}

Consider the model:

$$
y^{*}=0 . x_{1}+0 . x_{2}+\varepsilon
$$

where $x^{\top}=\left(x_{1}, x_{2}\right)^{\top}$ is a standard normal vector while $\varepsilon$ is independent of $x$ and uniformly distributed on $[-1 / 2,1 / 2]$. The true value of $\beta$ is $(0,0)^{\top}$. We assume that $y^{*}$ is observed by intervals defined as $\left(I_{k}=[-1 / 2+k / K ;-1 / 2+(k+1) / K], k=0 \ldots K-1\right)$.

The support function of the identified set $B$ is constant (see Appendix D.1):

$$
\delta^{*}(q \mid B)=\frac{2 \Delta}{\sqrt{2 \pi}}
$$

where $\Delta=\frac{1}{2 K}$. In other words, the identified set $B$ is a circle whose radius is $\frac{2 \Delta}{\sqrt{2 \pi}}$ (see Table 1 ).

We draw 1000 simulations in four different sample size experiments: $n=100,500,1000$ and 2500. We report results when the number of intervals, $K$, is equal to 2 as our results are robust when $K$ increases. The three quartiles as well as the mean of the distribution of the estimated support function at one angle are displayed in Table 1 although all angles give the same results. Even for small sample size, the identified set is well estimated and unsurprisingly, the interquartile interval decreases when the sample size increases.

With respect to the performance of test procedures, let $\beta^{0}=0$ be the center of $B$ and let $\beta^{r}$ a point on a ray such that the distance between 0 and $\beta^{r}$ is equal to $r$ times the value of the radius of $B$, a definition that is valid for any ray since set $B$ is a disk around the true value $\beta^{0}=0$. Point $\beta^{r}$ belongs to $B$ if and only if $r \leq 1$ and $\beta^{1}$ belongs to the frontier. For $r$ varying stepwise from 0 to 3 , we computed the rejection frequencies at a $5 \%$ level for the two tests developed in Section 4.2: Whether $\beta^{r}$ belongs to $B$ against the alternative that it does not (Test 1 ); Whether it belongs to the frontier of $B$ against the alternative that it does not (Test 2). Results are reported in Table 2. These results show that the size of the three tests is very accurate and remains very close to $5 \%$ even for $n=100$ and that the power of these tests is very good even in small samples.

\subsection{Smooth set with one supernumerary instrument}

The simulated model is as before except that the second explanatory variable $x_{2}$ is now generated by:

$$
x_{2}=\pi e_{2}+\sqrt{1-\pi^{2}} e_{3}
$$


where $\left(e_{2}, e_{3}\right)$ are i.i.d. standard normal variables. Moreover let $w=\nu e_{3}+\sqrt{1-\nu^{2}} e_{4}$ be another observed variable where $e_{4}$ is i.i.d. standard normal. Using general notations, we have $x=$ $\left(x_{1}, x_{2}\right)$ and $z=\left(x_{1}, e_{2}, w\right)$. Variables $x_{1}, e_{2}$ and $w$ are used for estimating set $B$ instead of $x_{1}$ and $x_{2}$ and we have therefore one supernumerary instrument. Note that parameter $\pi$ (respectively $\nu$ ) measures the strength of the correlation between $x_{2}$ and $e_{2}$ (respectively $x_{2}$ and $w$ ).

Setting $q=(\cos \theta, \sin \theta)^{\top}$, the support function can be expressed as (see Appendix D.2):

$$
\delta^{*}(q \mid B)=\frac{2 \Delta}{\sqrt{2 \pi}} \sqrt{\cos ^{2} \theta+\frac{\sin ^{2} \theta}{\pi^{2}+\nu^{2}\left(1-\pi^{2}\right)}}
$$

When $\nu=1$, set $B$ is the same as in the previous example because $x_{2}$ is a deterministic function of $e_{2}$ and $w$. Moreover when $\pi$ and $\nu$ are positive and strictly lower than 1 , there is some information loss due to the use of $e_{2}$ and $w$ instead of $x_{2}$ and set $B$ is stretched along the second axis (see Figure, Table 3).

As before, we draw 1000 simulations in four sample size experiments : $n=100,500,1000$ and 2500 with $\pi=\nu=0.9$. Table 3 displays descriptive statistics (Mean, quartiles) related to the distribution of the estimated support function at one angle. Table 4 displays the percentage of rejections for the tests for different points along the $\mathrm{x}$-axis. The line which corresponds to the frontier point $(r=1)$ is reported in bold. As before, there is no significative distortion when using supernumerary instruments in the estimation and test procedures.

\subsection{A set with kinks and faces}

In this experiment, the explanatory variable has mass points so that the identified set has exposed faces. Also its support is discrete so that the identified set has kinks. The simulated model is:

$$
y^{*}=\frac{1}{2}+\frac{x}{8}+\varepsilon
$$

where $x$ is equal to -1 with probability $\frac{1}{2}$ and to 1 with probability $\frac{1}{2}$ and where $\varepsilon$ is independent of $x$ and is uniformly distributed on $\left[-\frac{1}{4}, \frac{1}{4}\right]$. The true value of $\beta$ is $\left(\frac{1}{2}, \frac{1}{8}\right)^{\top}$. As before, we only observe $y^{*}$ by intervals $\left(I_{1}=\left[0, \frac{1}{2}\right]\right.$ and $\left.I_{2}=\left[\frac{1}{2}, 1\right]\right)$. The identified set $B_{2}$ can be shown to be the convex envelop of the four points $\left(\frac{3}{4}, \frac{1}{8}\right),\left(\frac{1}{2}, \frac{3}{8}\right),\left(\frac{1}{4}, \frac{1}{8}\right)$ and $\left(\frac{1}{2},-\frac{1}{8}\right)$ (see Appendix D.3). As in the previous example, we simulate 1000 draws for 4 sample sizes: 100, 500, 1000 and 2500 and the same conclusions concerning the estimation of the set remain valid here (see Table 6 in the Additional Appendix) 
One feature of this toy example is that, despite the presence of exposed faces, the additional term $\tau_{1}(q)$ in the asymptotic distribution of the support function (see Proposition 9) vanishes (see Appendix D.3) and we can apply the test procedures developed in the Gaussian case. We focus on the points belonging to the half-line starting from the central point $\beta^{*}=(1 / 2,1 / 8)$ and parallel to the x-axis. Like before, we index the points by $r$ the fraction of the distance to the frontier along this axis and $\beta^{1}=(3 / 4,1 / 8)$ the frontier point is now a kink of set $B$.

Table 5 displays the rejection rate for the test of the frontier for different values of $r$ (from 0.01 to 2) at a 5\%-level test (Table 7 in the Additional Appendix reports results for the test for the interior). In the first panel of columns (labeled $a_{n}=0$ ), we display results ignoring that there is a kink whereas by Proposition 10 we should be using perturbed programs $\left(a_{n}>0\right)$. Surprisingly, for the frontier test we do not overreject too much but we overreject for the interior test. In the second panel, we display the rejection rates using the perturbed program defined in Proposition 10 with $a_{n}=\frac{0.5}{n^{1 / 3}}$. Rejection rates are pretty close to the nominal size for both the frontier test and the interior test. Perturbing the program leads to correct quite efficiently for the presence of kinks except perhaps for very small sample sizes. Sample size properties can also be improved while estimating the variance with i.i.d. bootstrap techniques.

An additional general point is in order about small sample biases. We did not find in practice the sizeable small sample downward biases arising when minimizing an estimated function as discussed by Manski and Pepper (2009) for instance. Our set-up is arguably different since variable $q$ with respect to which the minimum is taken is not random and the standard error of the estimates varies slowly. We leave for further research the evaluation applied to our case of the correction approach recently advocated by Chernozhukov, Lee and Rosen (2009).

\section{Conclusion}

We develop in this paper a class of models defined by incomplete linear moment conditions and we provide examples of how this set up can be applied to economic data. In the most prominent one, the dependent variable in a linear model is censored by intervals. We present simple ways that lead to a sharp characterization of the identified sets. We generalize previous results about estimating such sets and we construct asymptotic tests for null hypotheses concerning the true value of the parameter of interest. These procedures are easy to implement and we can invert them and derive confidence regions for the parameter of interest. We also generalize the sim- 
ple setting of linear prediction using explanatory variables to the case in which supernumerary moment conditions are available. Specifically, we provide an extension to the usual Sargan test that can be performed using the asymptotic tests that we develop. Asymptotic properties of these generalized estimates are derived.

There remains many pending questions. Adapting our test procedure to the case in which the set has exposed faces is high on the agenda because exposed faces are a very common occurrence. Various other extensions were also out of the scope of this paper. First, some examples that we developed require more work in terms of estimation and asymptotic theory even if our set-up provides a building block to study the asymptotic properties of these estimates. For instance, for binary data with discrete or interval-valued regressors, the asymptotic properties of estimation would be the result of marrying the results of this paper with those of Lewbel (2000). Second, other examples about categorical data or two-sample combination need also some adaptation of the identification analysis.

Econometric assumptions can be questioned and extended. For simplicity, we focus on the case in which instruments and errors are not correlated. In structural settings, we would rather impose a stronger condition of mean independence between instruments and errors or even stronger of independence between instruments and errors. As is well known, mean independence (respectively independence) generates an infinite number of moment conditions given by the absence of correlation between any function of instruments and errors (respectively any function of errors). We presumably could use our framework by using only a finite number of moment conditions although the extension to the general case is worth pursuing. It also begs the question of the optimality of inference in the supernumerary restriction case and how it differs from the usual point-identified case.

Along a different vein, our setting remains global and semi-parametric. For non parametric estimation, it would be interesting to adapt our set-up to local approaches such as local linear regression. Other questions are open and seem worth pursuing. The gain of the direct approach that we used with respect to the approach followed by Chernozhukov et al. (2007) using a criterion is an interesting question. It is easy to write a criterion function using support functions (see Magnac and Maurin, 2008). It might be the case that our results help select the best criterion in the latter framework but this is left for future work.

Finally and more ambitiously, the deep foundation of our approach is a convexity argument. 
It indeed allows to replace the problem of identify a set in a very general space of sets by a problem which is finite dimensional since it requires to identify and estimate a function using finitely many parameters, the vectors of the unit sphere of $\mathbb{R}^{p}$. This approach can presumably be extended to any set identified problem when the set is convex. The problem of identifying the frontier of this set might be highly non linear although the real issue is to construct the support function, or the limits of the projection of the identified set in any direction $q$. Estimation and inference would likely follow from our arguments under adapted conditions. 


\section{REFERENCES}

Andrews, D.W.K., 1994, "Empirical Process Methods in Econometrics", eds R.Engle and D.McFadden, Handbook of Econometrics, IV:2247-2294, North Holland: Amsterdam.

Andrews, D.W.K., S., Berry and P. Jia, 2004, "Confidence Regions for Parameters in Discrete Games with Multiple Equilibria with an Application to Discount Chain Store Location", working paper.

Andrews, D.W.K. and P., Guggenberger, 2009, "Validity of Subsampling and Plug-in Asymptotic Inference for Parameters Defined by Moment Inequalities", Econometric Theory, 25:669-709.

Beresteanu, A. and F., Molinari, 2008, "Asymptotic Properties for a Class of Partially Identified Models", Econometrica, 76:763-814.

Blundell, R.W., A. Gosling, H. Ichimura and C. Meghir, 2007, "Changes in the Distribution of Male and Female Wages Accounting for Employment Composition Using Bounds", Econometrica, 75:323-363.

Bollinger, C.R., 1996, "Bounding mean regressions when a binary regressor is mismeasured", Journal of Econometrics, 73:387-399.

Bugni, F., 2009, "Bootstrap Inference in Partially Identified Models" working paper, Duke University.

Canay, I., 2009, 'EL Inference for Partially Identified Models: Large Deviations Optimality and Bootstrap Validity," Northwestern University.

Chernozhukov, V., H. Hong, E. Tamer, 2007, "Inference on Parameter Sets in Econometric Models", Econometrica, 75:1243-1284.

Chernozhukov, V., S. Lee and A.M. Rosen, 2009, "Intersection Bounds: Estimation and Inference", CEMMAP WP 19-09.

Chesher, A., 2005, "Non Parametric Identification under Discrete Variation", Econometrica, 73:1525-1550.

Ciliberto F. and E. Tamer, 2004, "Market structure and multiple equilibria in airline markets", forthcoming Econometrica.

Erickson, T., 1993, "Restricting Regression Slopes in the Errors in Variables Model by Bounding the Error Correlation", Econometrica, 61:959-69.

Fréchet, M., 1951, "Sur les tableaux de corrélation dont les marges sont données", Annales de l'Université de Lyon, III $^{\circ}$ Série Sci. A, 53-77.

Frisch, R., 1934, Statistical Confluence Analysis by Means of Complete Regression Systems, Oslo, Norway: University Institute of Economics.

Galichon A., and M., Henry, 2009, "A Test of Non-Identifying Restrictions and Confidence Regions for Partially Identified Parameters", forthcoming Journal of Econometrics.

Gini, C., 1921, "Sull'interpoliazone di una retta quando i valori della variabile indipendente sono affetti da errori accidentali", Metroeconomica, 1:63-82.

Haile P. and E.Tamer, 2003, "Inference with an Incomplete Model of English Auctions", Journal of Political Economy, 2003, 111:1-51.

Hoeffding, W., 1940, "Masstabinvariante Korrelationstheorie", Shriften des Mathematischen Instituts und des Instituts für Angewandte Mathematik der Universität Berlin, 5(3): 179-233.

Honoré, B. and A. Lleras-Muney, 2004, "Bounds in Competing Risks Models and the War on Cancer", Econometrica 74: 1675-1698.

Horowitz, J.L. and C.F. Manski, 1995, "Identification and Robustness with Contaminated and Corrupted Data", Econometrica, 63:281-302. 
Imbens, G., and C.F., Manski, 2004, "Confidence Intervals for Partially Identified Parameters", Econometrica, 72:1845-1859.

Klepper S., and E.E., Leamer, 1984, "Consistent Sets of Estimates for Regressions with Errors in all Variables", Econometrica, 52:163-183.

Leamer, E.E., 1987, "Errors in Variables in Linear Systems", Econometrica, 55(4): 893-909.

Lehmann, E.L. and J.P Romano, 2005, Testing Statistical Hypotheses, Springer: New York.

Lewbel, A., 2000, "Semiparametric Qualitative Response Model Estimation with Unknown Heteroskedasticity or Instrumental Variables", Journal of Econometrics, 97:145-77.

Magnac, T. and E. Maurin, 2007, "Identification and Information in Monotone Binary Models", Journal of Econometrics, 139:76-104.

Magnac, T. and E. Maurin, 2008, "Partial Identification in Monotone Binary Models: Discrete Regressors and Interval Data", Review of Economic Studies, 75:835-864.

Manski, C.F., 1989, "Anatomy of the Selection Problem", Journal of Human Resources, 24:343-60.

Manski, C.F., 2003, Partial Identification of Probability Distributions, Springer-Verlag: Berlin.

Manski, C.F., and J.V. Pepper, 2009, "More on monotone instrumental variables", The Econometrics Journal, 12:S200-216.

Manski, C.F., and E. Tamer, 2002, "Inference on Regressions with Interval Data on a Regressor or Outcome", Econometrica, 70:519-546.

McFadden, D., 1994, "Contingent Valuation and Social Choice", American Journal of Agricultural Economics, 76:689-708.

Pakes, A., J. Porter, K. Ho and J., Ishii, 2005, "Moment Inequalities and their Applications", working paper.

Piketty, T., 2005, "Top Income Share in the Long Run: An Overview", Journal of the European Economic Association, 3:1-11.

Ridder G. E. and R., Moffitt, 2007, "The econometrics of data combination", in J. J.Heckman and E. E. Leamer (Eds.), Handbook of Econometrics, Volume 6, North-Holland, Amsterdam.

Rockafellar, R.T., 1970, Convex Analysis, Princeton University Press: Princeton.

Romano J.P., and A.M. Shaikh, 2009, "Inference for Identifiable Parameters in Partially Identified Econometric Models", working paper.

Rosen A.M., 2008, "Confidence sets for partially identified parameters that satisfy a finite number of moment inequalities," Journal of Econometrics, 146:107-117.

Stoye, J., 2007, "Bounds on Generalized Linear Predictors with Incomplete Outcome Data", Reliable Computing, 13: 293-302.

Stoye, J., 2009, "More on Confidence Intervals for Partially Identified Parameters", Econometrica, 77:1299-1315.

van der Vaart, A.W., 1998, Asymptotic Statistics, Cambridge University Press: Cambridge.

Vazquez-Alvarez, R., B., Melenberg and A., van Soest, 2001, "Nonparametric bounds in the presence of item nonresponse, unfolding brackets, and anchoring", working paper.

White, H., 1999, Asymptotic Theory for Econometricians, Academic Press: San Diego. 


\section{Appendices}

\section{A Proofs in Section 2}

\section{A.1 Proof of Proposition 1}

(Necessity) Consider $\beta$ in $\mathbb{R}^{K}$ and assume that there is a latent random variable $\varepsilon$ uncorrelated with $x$ such that the latent variable $y^{*} \equiv x \beta+\varepsilon$ lies within the observed bounds, i.e., $x \beta+\varepsilon \in$ $[\underline{y} ; \bar{y}]$. Denoting $y=(\bar{y}+\underline{y}) / 2$ and using that $\varepsilon$ is uncorrelated with $x$, we have,

$$
E\left(x^{\top}(x \beta-y)\right)=E\left(x^{\top}\left(y^{*}-y\right)\right)=E\left(x^{\top} E\left(y^{*}-y \mid x\right)\right)
$$

We also have :

$$
-\frac{(\bar{y}-\underline{y})}{2} \leq y^{*}-y \leq \frac{(\bar{y}-\underline{y})}{2}
$$

which yields bounds on $u(x) \equiv E\left(y^{*}-y \mid x\right)$,

$$
-E\left(\frac{(\bar{y}-\underline{y})}{2} \mid x\right) \leq u(x) \leq E\left(\frac{(\bar{y}-\underline{y})}{2} \mid x\right)
$$

Setting $\Delta(x)=E\left(\frac{\bar{y}-\underline{y}}{2} \mid x\right)$, there thus exists a measurable $u(x) \in[-\Delta(x), \Delta(x)]$ such that $E\left(x^{\top}(x \beta-y)\right)=E\left(x^{\top} u(x)\right)$.

(Sufficiency) Conversely, let us assume that there exists $u(x)$ in $[-\Delta(x), \Delta(x)]$ such that

$$
E\left(x^{\top}(x \beta-y)\right)=E\left(x^{\top} u(x)\right) .
$$

We are going to construct a random variable $\varepsilon$ which is uncorrelated with $x$ and which is such that $y^{*} \equiv x \beta+\varepsilon$ lies within the observed bounds.

First, consider $\lambda$ a random variable whose support is $[0,1]$, which is independent of $\underline{y}$ and $\bar{y}$ and whose conditional mean given $x$ is:

$$
E(\lambda \mid x)=\frac{1}{2} \frac{u(x)}{\Delta(x)}+\frac{1}{2}
$$

Second, define $\varepsilon$ as :

$$
\varepsilon=-x \beta+(1-\lambda) \underline{y}+\lambda \bar{y}
$$

By construction, $y^{*} \equiv x \beta+\varepsilon$ is consistent with the observed censoring mechanism i.e. $y^{*} \in$ $[\underline{y} ; \bar{y}]$. Let us prove that $\varepsilon$ is also uncorrelated with $x$. Consider, for almost any $x$,

$$
\begin{aligned}
E(y \mid x)-E(x \beta+\varepsilon \mid x) & =E\left(\frac{(\bar{y}+\underline{y})}{2} \mid x\right)-E((1-\lambda) \underline{y}+\lambda \bar{y} \mid x) \\
& =E\left((1-2 \lambda) \frac{(\bar{y}-\underline{y})}{2} \mid x\right)=E((1-2 \lambda) \mid x) E\left(\frac{(\bar{y}-\underline{y})}{2} \mid x\right) \\
& =E\left(-\frac{u(x)}{\Delta(x)} \Delta(x) \mid x\right)=-u(x) .
\end{aligned}
$$

where we used that $\lambda$ is independent of $\underline{y}$ and $\bar{y}$. Therefore, we have $E(\varepsilon \mid x)=E(y-x \beta \mid x)+$ $u(x)$, which implies:

$$
E\left(x^{\top} \varepsilon\right)=E\left(x^{\top}(y-x \beta)\right)+E\left(x^{\top} u(x)\right)=-E\left(x^{\top} u(x)\right)+E\left(x^{\top} u(x)\right)=0 .
$$

using the moment condition (A.1) involving $y, \beta$ and $u(x)$. 


\section{B Proofs in Section 3}

\section{B.1 Proof of Proposition 2}

The support function in direction $q \in \mathbb{S}$ is obtained as the supremum of the expression

$$
q^{\top} \beta=E\left(z_{q}(y+u(z))\right),
$$

where $u(z)$ varies in $[\Delta(z), \bar{\Delta}(z)]$. The supremum of the scalar $E\left(z_{q} u(z)\right)$ is obtained by setting $u(z)$ to its maximum (resp. minimum) value when $z_{q}$ is positive (resp. negative) and by setting $u(z)$ to any value when $z_{q}$ is equal to 0 . It yields a set of "supremum" functions:

$$
u_{q}(z)=\underline{\Delta}(z)+(\bar{\Delta}(z)-\underline{\Delta}(z)) \mathbf{1}\left\{z_{q}>0\right\}+\boldsymbol{\Delta}^{*}(z) \mathbf{1}\left\{z_{q}=0\right\}
$$

where $\Delta^{*}(z) \in[\underline{\Delta}(z), \bar{\Delta}(z)]$. Note that $u_{q}(z)$ is unique (a.e. $P_{z}$ ) if $\operatorname{Pr}\left(z_{q}=0\right)=0$. From now on, the uniqueness of $u_{q}(z)$ should always be understood as "almost everywhere $P_{z}$ ".

Recall that by equation (3), $E(\bar{y}-y \mid z)=\bar{\Delta}(z), E(y-y \mid z)=\Delta(z)$, so that the support function or the supremum of (B.2) is equal to:

$$
\delta^{*}(q \mid B)=E\left(z_{q} w_{q}\right),
$$

where:

$$
w_{q}=\underline{y}+\mathbf{1}\left\{z_{q}>0\right\}(\bar{y}-\underline{y}) .
$$

Note that the term $\Delta^{*}(z)$ in $u_{q}(z)$ disappears because it is multiplied within the second expectation by $z_{q}$ which is equal to 0 at these values. It implies, as expected, that $\delta^{*}(q \mid B)$ is unique even though $u_{q}(z)$ is not.

Furthermore, when $\operatorname{Pr}\left(z_{q}=0\right)>0$, since $\Delta^{*}(z)$ varies in $[\underline{\underline{ }}(z), \bar{\Delta}(z)]$, the functions $u_{q}(z)$ defined by equation (B.3) generate all the points $\beta=\left(E\left(z^{\top} x\right)\right)^{-1} E\left(z^{\top}\left(y+u_{q}(z)\right)\right)$ which belong to the tangent space to $B$ whose outer-pointing normal vector is $q$ (an exposed face in the vocabulary used in the next Proposition).

If we select the specific value of $u_{q}(z)$ that corresponds to $\Delta^{*}(z)=0$, we get the particular value of $\beta$ :

$$
\beta_{q}=\left(E\left(z^{\top} x\right)\right)^{-1} E\left(z^{\top} w_{q}\right),
$$

and, by definition:

$$
\delta^{*}(q \mid B)=q^{\top} \beta_{q}
$$

Finally, the interior of $B$ is not empty, if we can prove that, for any $q \in \mathbb{S}$,

$$
\sup _{\beta \in B} q^{\top} \beta>\inf _{\beta \in B} q^{\top} \beta
$$

or equivalently that:

$$
\delta^{*}(q \mid B)>-\delta^{*}(-q \mid B)
$$

Start from consequences of definitions:

$$
z_{q}=q^{\top}\left(E\left(z^{\top} x\right)\right)^{-1} z^{\top}=-z_{-q}, w_{q}-w_{-q}=(\bar{y}-\underline{y})\left(\mathbf{1}\left\{z_{q}>0\right\}-\mathbf{1}\left\{z_{q}<0\right\}\right),
$$

so that:

$$
\begin{aligned}
\delta^{*}(q \mid B)+\delta^{*}(-q \mid B) & =E\left(\left|z_{q}\right|(\bar{y}-\underline{y})\right) \\
& =E\left(\left|z_{q}\right| E((\bar{y}-\underline{y}) \mid z)\right) \\
& =E\left(\left|z_{q}\right|(\bar{\Delta}(z)-\underline{\Delta}(z))\right)>0
\end{aligned}
$$


because of equation (3) and $\left|z_{q}\right|>0$ with positive probability because of the full rank assumption in R.iii.

This quantity $\delta^{*}(q \mid B)+\delta^{*}(-q \mid B)$ is the width of $B$ in direction $q$, and by using the same argument:

$$
\min _{q \in \mathbb{S}}\left(\delta^{*}(q \mid B)+\delta^{*}(-q \mid B)\right)>0
$$

since $\mathbb{S}$ is compact.

\section{B.2 Proof of Lemma 3}

We use the expression derived in Proposition 2:

$$
\delta^{*}(q \mid B)=E\left(z_{q} w_{q}\right)=E\left(z_{q} \underline{y}\right)+E\left(z_{q} \mathbf{1}\left\{z_{q}>0\right\}(\bar{y}-\underline{y})\right) .
$$

First of all, the support function of a convex set is convex and therefore is differentiable except at a countable number of directions $q$ denoted $D_{f}$. In this proof, we characterize $D_{f}$. It corresponds to the set of directions that are orthogonal to the exposed faces of $B$. We also characterize kink points of set $B$.

Characterization of $D_{f} \quad$ The first term on the RHS of equation (B.4) is linear in $q$ since (see the previous proof) :

$$
z_{q}=z\left(E\left(x^{\top} z\right)\right)^{-1} q
$$

and thus is continuously differentiable on $\mathbb{S}$. The second term can be written as:

$$
\psi(q)=E\left(z^{*} q \mathbf{1}\left\{z^{*} q>0\right\}\right)
$$

where $z^{*}=z\left(E\left(x^{\top} z\right)\right)^{-1}(\bar{y}-y)$. The set of points $D_{f}$ is the set of points where $\psi(q)$ is not differentiable.

Fix $q \in \mathbb{S}$. For any $t \in \mathbb{S}$ :

$$
\psi(t)-\psi(q)=E\left(\left(z^{*}(t-q) \mathbf{1}\left\{z^{*} q>0\right\}\right)+E\left(\left(z^{*} t\left(\mathbf{1}\left\{z^{*} t>0\right\}-\mathbf{1}\left\{z^{*} q>0\right\}\right),\right.\right.\right.
$$

so that:

$$
\psi(t)-\psi(q)-E\left(z^{*} \mathbf{1}\left\{z^{*} q>0\right\}\right)(t-q)=E\left(\left(z^{*} t\left(\mathbf{1}\left\{z^{*} t>0\right\}-\mathbf{1}\left\{z^{*} q>0\right\}\right) .\right.\right.
$$

Points of non differentiability depends on the expression in the RHS. It is the sum of three terms

$$
\begin{aligned}
& A_{1}=E\left(z^{*} t \mathbf{1}\left\{z^{*} t>0, z^{*} q<0\right\}\right), \\
& A_{2}=-E\left(z^{*} t \mathbf{1}\left\{z^{*} q>0, z^{*} t \leq 0\right\}\right) \\
& A_{3}=E\left(z^{*} t \mathbf{1}\left\{z^{*} q=0, z^{*} t>0\right\}\right)
\end{aligned}
$$

Regarding $A_{1}$ and $A_{2}$, when $z^{*} t>0$ and $z^{*} q<0$, we have,

$$
0<z^{*} t=z^{*}(t-q)+z^{*} q<z^{*}(t-q),
$$

whereas when $z^{*} q>0$ and $z^{*} t \leq 0$, we have,

$$
z^{*}(t-q)<z^{*} t \leq 0 .
$$


Hence, we get,

$$
\begin{aligned}
& 0 \leq\left|A_{1}\right| \leq E\left(\left\|z^{*}\right\|\right)\|t-q\| \operatorname{Pr}\left(z^{*} t>0, z^{*} q<0\right) \\
& 0 \leq\left|A_{2}\right| \leq E\left(\left\|z^{*}\right\|\right)\|t-q\| \operatorname{Pr}\left(z^{*} q>0, z^{*} t \leq 0\right) .
\end{aligned}
$$

As $\operatorname{Pr}\left(z^{*} t>0, z^{*} q<0\right)=\operatorname{Pr}\left(z^{*}(t-q)>-z^{*} q>0\right)$ we have $\lim _{t \rightarrow q} \operatorname{Pr}\left(z^{*} t>0, z^{*} q<0\right)=$ 0 . Similarly, $\lim _{t \rightarrow q} \operatorname{Pr}\left(z^{*} q>0, z^{*} q \leq 0\right)=0$, so that these inequalities imply:

$$
A_{1}=o(\|t-q\|) \text { and } A_{2}=o(\|t-q\|),
$$

since R.iii implies that $E\left(\left\|z^{*}\right\|\right)$ is bounded.

Regarding the last term $A_{3}$, note that in the case in which $\operatorname{Pr}\left(z^{*} q=0\right)=0$, we have $A_{3}=0$ and thus $\psi(q)$ is differentiable at $q$. Its gradient is given by equation (B.5):

$$
\nabla_{q} \psi(q)=E\left(z^{*} \mathbf{1}\left\{z^{*} q>0\right\}\right)
$$

and is continuous in $q$.

Consider now the case in which $\operatorname{Pr}\left(z^{*} q=0\right)>0$. When $t \rightarrow q$, both in $\mathbb{S}$, define:

$$
t-q=h s+o(h)
$$

where $h=\|t-q\|$ and $s \in \mathbb{S}, s^{T} q=0$. We have

$$
\begin{aligned}
A_{3} & =E\left(z^{*} t \mathbf{1}\left\{z^{*} q=0, z^{*} t>0\right\}\right)=E\left(z^{*}(t-q) \mathbf{1}\left\{z^{*} q=0, z^{*}(t-q)>0\right\}\right) \\
& =\operatorname{Pr}\left(z^{*} q=0\right) E\left(z^{*} s 1\left\{z^{*} s \geq 0\right\} \mid z^{*} q=0\right) h+o(h)
\end{aligned}
$$

It follows that $\psi$ has different gradients in different directions $s$, which depend on the term,

$$
E\left(z^{*} 1\left\{z^{*} s \geq 0\right\} \mid z^{*} q=0\right) .
$$

This vector is constant for any $s$ if and only if (using $s$ and $-s$ ):

$$
E\left(\left|z^{*} s\right| \mid z^{*} q=0\right)=0 .
$$

The support of $z^{*}$ conditional on $\left(z^{*} q=0\right)$ boils down to $\{0\}$, that is, if and only if the conditional support of $z$ itself is $\{0\}$. This case is excluded by condition (R.ii) and therefore, function $\psi(q)$ is not differentiable.

Overall, the points of non differentiability of the support function are directions $q$ such that $\operatorname{Pr}\left(z^{*} q=0\right)=\operatorname{Pr}\left(z_{q}=0\right)>0$. There can be no more than a countable number of such points.

Exposed faces Using Lemma 3 we obtain for any $q$ which does not belong to $D_{f}$ :

$$
\frac{\partial \delta^{*}(q \mid B)}{\partial q^{\top}}=\left(E\left(z^{\top} x\right)\right)^{-1} E\left(z^{\top} w_{q}\right)=\beta_{q}
$$

As $\delta^{*}(q \mid B)=q^{\top} \beta_{q}$, and $\beta_{q} \in \arg \max _{\beta \in B}\left(q^{\top} \beta\right)$, this result is a disguise of the envelope theorem.

Assume now that $B$ has an exposed face $B_{f}$. By definition, $B_{f}$ is the intersection of $B$ with one of its supporting hyperplane $H_{f}$ which is not reduced to a singleton. If $q_{f}$ denotes the vector orthogonal to $H_{f}$, we have for any $\beta_{f}$ in $B_{f}$ :

$$
\delta^{*}\left(q_{f} \mid B\right)=q_{f}^{\top} \beta_{f}
$$


which means (see equation (B.3)) that there exists $\Delta_{f}^{*}(z)$ in $[\underline{\underline{\Delta}}(z), \bar{\Delta}(z)]$ such that:

$$
\begin{aligned}
\beta_{f} & =\beta_{q_{f}}+\left(E\left(z^{\top} x\right)\right)^{-1} E\left(z^{\top} \Delta_{f}^{*}(z) \mathbf{1}\left\{z_{q_{f}}=0\right\}\right) \\
& =\beta_{q_{f}}+\left(E\left(z^{\top} x\right)\right)^{-1} E\left(z^{\top} \Delta_{f}^{*}(z) \mid z_{q_{f}}=0\right) \operatorname{Pr}\left(z_{q_{f}}=0\right) .
\end{aligned}
$$

For the set of all $\beta_{f}$ not to be reduced to the singleton $\left\{\beta_{q_{f}}\right\}$, we clearly need $\operatorname{Pr}\left(z_{q}=0\right)>0$ and the conditional support of $z$ not reduced to $\{0\}$.

Conversely, suppose that there exists a direction $q$ such that $\operatorname{Pr}\left(z_{q}=0\right)>0$ and suppose that the conditional support of $z$ is not reduced to $\{0\}$. Denote $\beta_{q}=\left(E\left(z^{\top} x\right)\right)^{-1} E\left(z^{\top} w_{q}\right)$ and $H_{q}$ the supporting hyperplane at $\beta_{q}$ orthogonal to $q$. Consider the set $B_{f}$ of all $\beta_{f}$ such that there exists $\Delta_{f}^{*}(z)$ in $[\underline{\Delta}(z), \bar{\Delta}(z)]$ such that:

$$
\begin{aligned}
\beta_{f} & =\beta_{q}+\left(E\left(z^{\top} x\right)\right)^{-1} E\left(z^{\top} \boldsymbol{\Delta}_{f}^{*}(z) \mathbf{1}\left\{z_{q}=0\right\}\right) \\
& =\beta_{q}+\left(E\left(z^{\top} x\right)\right)^{-1} E\left(z^{\top} \boldsymbol{\Delta}_{f}^{*}(z) \mid z_{q}=0\right) \operatorname{Pr}\left(z_{q}=0\right) .
\end{aligned}
$$

$B_{f}$ is clearly included in $B \cap H_{q}$.Also, as $\operatorname{Pr}\left(z_{q}=0\right)$ is positive and the conditional support of $z$ is not reduced to $\{0\}$, the second term in the RHS is itself non zero for at least some $\Delta_{f}^{*}(z)$, which implies that $B_{f}$ is not reduced to the singleton $\left\{\beta_{q}\right\}$ and that $B$ has an exposed face.

Kinks Assume that $\operatorname{Pr}\left(z_{q}=0\right)=0$ so that the support function is differentiable and $B$ is strictly convex. Even in this case, it is still possible to observe points $\beta_{k} \in \partial B$ where the tangent space is not unique (kinks), i.e., points of the surface such that there exist at least two distinct vectors $q$ and $r(r \neq q)$ satisfying $\beta_{k}=\beta_{q}=\beta_{r}$. When there exist such points, the relationship between directions of the unit sphere and points of the frontier of $B$ is not one-to-one anymore. It complicates the construction of testing procedures (as shown in Section 4) and this is the reason why it is useful to characterize set-ups where $B$ has kinks. We have,

$$
\begin{aligned}
\beta_{q} & =\beta_{r}, \\
& \Leftrightarrow E\left(z^{\top} w_{q}\right)=E\left(z^{\top} w_{r}\right) \\
& \Leftrightarrow E\left(z^{\top}(\bar{y}-\underline{y})\left(\mathbf{1}\left\{z_{q}>0\right\}-\mathbf{1}\left\{z_{r}>0\right\}\right)\right)=0 \\
& \Leftrightarrow E\left(z^{\top}(\bar{y}-\underline{y})\left(\mathbf{1}\left\{z_{q}>0, z_{r}<0\right\}-\mathbf{1}\left\{z_{q}<0, z_{r}>0\right\}\right)\right)=0,
\end{aligned}
$$

the last equation holding because we have assumed that $\operatorname{Pr}\left(z_{q}=0\right)=0$.

Premultiplying last equation by $q^{\top}\left(E\left(z^{\top} x\right)\right)^{-1}$, we get,

$$
\beta_{q}=\beta_{r} \Rightarrow E\left(z_{q}(\bar{y}-\underline{y})\left(\mathbf{1}\left\{z_{q}>0, z_{r}<0\right\}-\mathbf{1}\left\{z_{q}<0, z_{r}>0\right\}\right)\right)=0 .
$$

Given that the term within the expectation is necessarily non negative, the fact that the expectation is zero implies necessarily

$$
\operatorname{Pr}\left\{z_{q}>0, z_{r}<0\right\}=\operatorname{Pr} \mathbf{1}\left\{z_{q}<0, z_{r}>0\right\}=\mathbf{0}
$$

It follows that the existence of $q$ and $r(r \neq q)$ satisfying the latter condition is not only sufficient, but also necessary for the existence of kinks. 


\section{B.3 Proof of Lemma 4}

We have already proven that conditions (9) and (10) are necessary, we want to prove that they are sufficient. Specifically, we suppose that conditions (9) and (10) hold true and we want to prove that

$$
E\left(z^{\top}(x \beta-(y+u(z)))\right)=0
$$

To prove this, we are going to show that $z$ can be written as a linear combination of $z_{F}$ and $z_{H}$. Note first that :

$$
z_{F}=z\left(E\left(z^{\top} z\right)\right)^{-1} E\left(z^{\top} x\right)\left[E\left(x^{\top} z\right)\left(E\left(z^{\top} z\right)\right)^{-1} E\left(z^{\top} x\right)\right]^{-1 / 2}=z\left(E\left(z^{\top} z\right)\right)^{-1 / 2} Q_{F},
$$

where $Q_{F}$ is a $[m, p]$ matrix of rank $p$ satisfying $Q_{F}^{\top} Q_{F}=I_{p}$ (where $I_{p}$ is the identity matrix of dimension $p)$. Second, denoting $A=\left(\begin{array}{c}0_{p, m-p} \\ I_{m-p, m-p}\end{array}\right)$ the $(m, m-p)$ selection matrix, the definition of $z^{s}$ implies,

$$
z^{s}=z A=z\left(E\left(z^{\top} z\right)\right)^{-1 / 2} A^{s}
$$

where $A^{s}=\left(E\left(z^{\top} z\right)\right)^{1 / 2} A$.

Denoting $P_{F}=Q_{F} Q_{F}^{\top}$ and $P_{H}=I_{m}-P_{F}, P_{F}$ and $P_{H}$ are two orthogonal projections and we have,

$$
\zeta^{s}=z^{s}-z_{F} E\left(z_{F}^{\top} z^{s}\right)=z\left(E\left(z^{\top} z\right)\right)^{-1 / 2}\left(I_{m}-P_{F}\right) A^{s}=z\left(E\left(z^{\top} z\right)\right)^{-1 / 2} P_{H} A^{s},
$$

which implies,

$$
z_{H}=\zeta^{s}\left(\zeta^{s \top} \zeta^{s}\right)^{-1 / 2}=z\left(E\left(z^{\top} z\right)\right)^{-1 / 2} P_{H} A^{s}\left(A^{s^{\top}} P_{H} A^{s}\right)^{-1 / 2}=z\left(E\left(z^{\top} z\right)\right)^{-1 / 2} Q_{H},
$$

where $Q_{H}=P_{H} A^{s}\left(A^{s \top} P_{H} A^{s}\right)^{-1 / 2}$ is a matrix of dimension $[m, m-p]$ of rank $(m-p)$ satisfying $Q_{H}^{\top} Q_{H}=I_{m-p}$ and $Q_{F}^{\top} Q_{H}=0_{p, m-p}$.

Overall, the relationship between $\left(z_{F}, z_{H}\right)$ and $z$ boils down to,

$$
\left(z_{F}, z_{H}\right)=z\left(E\left(z^{\top} z\right)\right)^{-1 / 2}\left(Q_{F}, Q_{H}\right)=z\left(E\left(z^{\top} z\right)\right)^{-1 / 2} Q
$$

where the $(m, m)$ matrix $Q=\left(Q_{F}, Q_{H}\right)$ satisfies $Q^{\top} Q=I_{m}$ and hence has full rank. Hence $z$ may be written $\left(z_{F}, z_{H}\right) Q^{-1}\left(E\left(z^{\top} z\right)\right)^{1 / 2}$ i.e., a linear combination of $z_{F}$ and $z_{H}$. In such a case, conditions (9) and (10) implies

$$
E\left(z^{\top}(x \beta-(y+u(z)))\right)=0
$$

which finishes the proof.

We can now show that the choice of $z^{s}$ is without loss of generality. Suppose that $z_{H}$ associated with a given subset of supernumerary instruments $z^{s}$ satisfies condition (10). Then, $B$ is non empty because condition (10) is sufficient. Yet, if $B$ is non empty and since condition (10) is necessary, condition (10) is necessarily satisfied by any other subset of $(m-p)$ instruments (say $z_{H}^{*}$ ) constructed from an alternative $z^{* s}$ satisfying the same condition as $z^{s}$. Overall, because condition (10) is both necessary and sufficient for the condition that $B$ is not empty, when it is satisfied by a given subset of supernumerary instruments, it is necessarily satisfied by any alternative subsets. 
There is another interesting way to see why restrictions involved by condition (10) are invariant to the choice of the specific subset of supernumerary instruments. As discussed above $z_{H}$ can be written as $z\left(E\left(z^{\top} z\right)\right)^{-1 / 2} Q_{H}$ where the $m-p$ columns of matrix $Q_{H}$ are an orthonormal basis of the kernel of the orthogonal projection onto $x(z)$. Changing one specific subset of supernumerary instruments $z_{H}$ into an alternative subset $z_{H}^{*}$ boils down to moving from one orthonormal basis $Q_{H}$ to an alternative basis $Q_{H}^{*}$ (i.e., to $Q_{H}^{*}=Q_{H} R$, where $R$ is an orthogonal matrix). In other words, for any $z_{H}^{*}$ satisfying the same conditions as $z_{H}$, there exists necessarily an orthogonal matrix $R$ (with $R=Q_{H}^{\top} Q_{H}^{*}$ ) such that $z_{H}^{*}=z_{H} R$. This basic linear relationship between all possible subsets of supernumerary instruments implies that when linear moment condition (10) is satisfied by a given subset it is necessarily satisfied by any alternative one.

\section{B.4 Proof of Proposition 6}

We assume that the Sargan condition (as given by Proposition 5) is satisfied so that the intersection of the set $B_{U}$ and the hyperplane, $\gamma=0$, is not empty. Both sets $\{\gamma=0\}$ and $B_{U}$ are convex. The support function of $B_{U}$ is $\delta^{*}\left(x_{1}^{*} \mid B_{U}\right)$ where $x_{1}^{*}=\left(q_{1}, \lambda_{1}\right)$. The support function of $\{\gamma=0\}$ is as follows if $x_{2}^{*}=\left(q_{2}, \lambda_{2}\right)$ :

$$
\delta^{*}\left(x_{2}^{*} \mid\{\gamma=0\}\right)=\sup _{(\beta, \gamma) \in\{\gamma=0\}} \beta^{\top} q_{2}+\gamma^{\top} \lambda_{2}=\sup _{\beta \in \mathbb{R}^{p}} \beta^{\top} q_{2}=\left\{\begin{array}{c}
0 \text { if } q_{2}=0 \\
+\infty \text { if } q_{2} \neq 0
\end{array}\right.
$$

Corollary 16.4.1 page 146 of Rockafellar (1970) states that the support function $\delta^{*}\left(x^{*}\right)$, where $x^{*}=(q, \lambda)$, of the intersection of two convex sets such that their relative interiors ${ }^{15}$ have one point in common, can be written:

$$
\delta^{*}\left(x^{*} \mid B_{U} \cap\{\gamma=0\}\right)=\inf _{\left(x_{1}^{*}, x_{2}^{*}\right): x_{1}^{*}+x_{2}^{*}=x^{*}}\left(\delta^{*}\left(x_{1}^{*} \mid B_{U}\right)+\delta^{*}\left(x_{2}^{*} \mid\{\gamma=0\}\right)\right)
$$

and the infimum is attained. When the hyperplane $\{\gamma=0\}$ is not tangent to $B_{U}$ and their intersection is not empty, their relative interiors have all the points of the relative interior of their intersection in common, and we have:

$$
\left.\left.\delta^{*}((q, \lambda) \mid B)=\inf _{\left(\lambda_{1}, \lambda_{2}\right): \lambda_{1}+\lambda_{2}=\lambda} \delta^{*}\left(\left(q, \lambda_{1}\right) \mid B_{U}\right)\right)=\inf _{\lambda_{1}} \delta^{*}\left(\left(q, \lambda_{1}\right) \mid B_{U}\right)\right)
$$

as the RHS is independent of $\lambda_{2}$ and $\lambda$. Furthermore, the infimum in $\lambda_{1}$ is attained.

On the other hand, when the hyperplane $\{\gamma=0\}$ is tangent to $B_{U}$, the relative interiors have no points in common. The same corollary 16.4.1 of Rockafellar (1970) states that we should replace equation (B.6) by its closure and the infimum is not necessarily attained. The condition under which the hyperplane $\{\gamma=0\}$ is tangent to $B_{U}$, is obtained when the origin point belongs to the frontier of $B_{\text {Sargan }}$ (see Section 3.2.3). When it is assumed that the support function is differentiable, there are no exposed faces and the tangency of the hyperplane $\{\gamma=0\}$ to $B_{U}$ results in one single point. Set $B$ is reduced to a singleton and is not a proper set any longer.

\footnotetext{
${ }^{15}$ Let the smallest affine set containing $C$, be af $f(C)$. Let $B(x, \varepsilon)$ be the ball centered at $x$ and of diameter $\varepsilon / 2$. The relative interior of a set $C$ is defined as:

$$
\operatorname{ri}(C)=\{x \in \operatorname{aff}(C) ; \exists \varepsilon>0, B(x, \varepsilon) \cap \operatorname{aff}(C) \subset C\}
$$
}




\section{B.5 The Construction of $B_{U}$}

Let $s=(q, \lambda)$ be the direction used for estimating $B_{U}, \lambda$ being the components relative to the variables $z_{H}$. By definition of $B_{U}$, we have that:

$$
\left[\begin{array}{l}
\beta \\
\gamma
\end{array}\right]=\left[E\left(z^{\top} x\right): E\left(z^{\top} z_{H}\right)\right]^{-1} E\left[z^{\top}(y+u(z))\right] .
$$

The support function of $B_{U}$ is as in Proposition 2

$$
\delta^{*}\left(s \mid B_{U}\right)=E\left(z_{s} w_{s}\right),
$$

where $z_{s}=s^{\top} \Omega^{\top} z^{\top}, \quad w_{s}=\underline{y}+(\bar{y}-\underline{y}) \mathbf{1}\left\{z_{s}>0\right\}$ and

$$
\Omega=\left[E\left(z^{\top} x\right): E\left(z^{\top} z_{H}\right)\right]^{-1} .
$$

The last matrix is well defined because of the rank conditions R.ii and Appendix B.3.

The invariance of this construction to the specific choice of $z_{H}$ follows the same argument as before. Write:

$$
z_{H} \gamma=z_{H} Q Q^{\top} \gamma, \lambda^{\top} \gamma=\lambda^{\top} Q Q^{\top} \gamma
$$

for any arbitrary orthogonal matrix $Q$. The solution is thus invariant to the choice of $Q$ provided that $\left(z_{H}, \gamma, \lambda\right)$ is changed into $\left(z_{H} Q, Q^{\top} \gamma, Q^{\top} \lambda\right)$. Minimizing with respect to $\lambda$ or $Q^{\top} \lambda$ is equivalent.

\section{Proofs in Section 4}

We denote $M$ a generic majorizing constant.

\section{C.1 Proof of Proposition 9}

We use that:

$$
\delta^{*}(q \mid B)=E\left(z_{q} w_{q}\right)=q^{\top}\left(E\left(z^{\top} x\right)\right)^{-1} E\left(z^{\top} w_{q}\right)=q^{\top} \Sigma^{\top} E\left(z^{\top} w_{q}\right) .
$$

where $\Sigma=E\left(x^{\top} z\right)^{-1}$. The estimator that we consider is:

$$
\hat{\delta}_{n}^{*}(q \mid B)=\frac{1}{n} \sum z_{n, q i} w_{n, q i}
$$

where:

$$
\begin{gathered}
z_{n, q i}=q^{\top} \cdot \hat{\Sigma}_{n}^{\top} z_{i}^{\top}, \\
w_{n, q i}=\underline{y}_{i}+\mathbf{1}\left\{z_{n, q i}>0\right\}\left(\bar{y}_{i}-\underline{y}_{i}\right),
\end{gathered}
$$

where $\hat{\Sigma}_{n}$ is an estimate of $\Sigma$.

Define $\|\Sigma\|=\operatorname{Tr}(\Sigma)$ and choose $M$ arbitrarily such that $M>\operatorname{Tr}(\Sigma)$. We now show that we can construct an estimate of $\Sigma$ satisfying $\left\|\hat{\Sigma}_{n}\right\| \leq M$. Define $\hat{\Sigma}_{n}^{u}$ the sample analog of $\Sigma$ :

$$
\hat{\Sigma}_{n}^{u}=\left(\frac{1}{n} \sum x_{i}^{\top} z_{i}\right)^{-1}
$$


and define $\hat{\Sigma}_{n}$, the estimate of $\Sigma$, as:

$$
\left\{\begin{array}{cc}
\hat{\Sigma}_{n}=\hat{\Sigma}_{n}^{u} & \text { if }\left\|\hat{\Sigma}_{n}^{u}\right\| \leq M \\
\hat{\Sigma}_{n}=\hat{\Sigma}_{n}^{u}\left(\frac{M}{\left\|\hat{\Sigma}_{n}^{u}\right\|}\right) & \text { if not. }
\end{array}\right.
$$

The element $\left(q, \hat{\Sigma}_{n}\right)$ always belongs to the bounded set $\Theta=\mathbb{S} \times\{\|\Sigma\| \leq M\}$. Under the conditions of Proposition $8, \hat{\Sigma}_{n}$ is almost surely consistent to $\Sigma$ :

$$
\lim _{n \rightarrow \infty} \operatorname{Pr}\left(\sup _{n>N}\left\|\hat{\Sigma}_{n}-\Sigma\right\| \geq \varepsilon\right)=0 .
$$

Under the conditions of Proposition $9, \hat{\Sigma}_{n}^{u}$ and $\hat{\Sigma}_{n}$ are asymptotically equivalent:

$$
\sqrt{n}\left(\hat{\Sigma}_{n}-\hat{\Sigma}_{n}^{u}\right) \underset{n \rightarrow \infty}{\stackrel{P}{\rightarrow}} 0
$$

and the estimate is asymptotically normal:

$$
\sqrt{n}\left(\operatorname{vec}\left(\hat{\Sigma}_{n}^{\top}-\Sigma^{\top}\right)\right) \Longrightarrow N(0, W) .
$$

We proceed in two steps. As the first step is simple, we give the proof consistency and asymptotic normality at the same time.

\section{C.1.1 Consistency and Asymptotic Normality: $\Sigma$ is known}

Suppose that $\Sigma$ is known and denote:

$$
z_{q i}=z_{i} \Sigma q, w_{q i}=\underline{y}_{i}+\mathbf{1}\left\{z_{q i}>0\right\}\left(\bar{y}_{i}-\underline{y}_{i}\right) .
$$

Consider function $f_{\theta}$ indexed by $\theta=(q, \Sigma) \in \Theta$ from the the support of $\left(z_{i}, \underline{y}_{i}, \bar{y}_{i}\right)$ to $\mathbb{R}$ such that:

$$
f_{\theta}\left(z_{i}, \underline{y}_{i}, \bar{y}_{i}\right)=z_{q i} w_{q i}=q^{\top} \Sigma^{\top} z_{i}^{\top}\left(\underline{y}_{i}+\mathbf{1}\left\{q^{\top} \Sigma^{\top} z_{i}^{\top}>0\right\}\left(\bar{y}_{i}-\underline{y}_{i}\right)\right) .
$$

Note that $\mathcal{F}=\left\{f_{\theta} ; \theta \in \Theta\right\}$ is a parametric class and is indexed by a parameter $\theta$ lying in a bounded set $\Theta$.

As the proof of Lemma 3 shows, this function is convex in $\Sigma q$ and therefore Lipschitzian:

$$
\begin{aligned}
\left|f_{\theta_{1}}\left(z_{i}, \underline{y}_{i}, \bar{y}_{i}\right)-f_{\theta_{2}}\left(z_{i}, \underline{y}_{i}, \bar{y}_{i}\right)\right| & \leq \max \left(\left\|z_{i}^{\top} \underline{y}_{i}\right\|,\left\|z_{i}^{\top} \bar{y}_{i}\right\|\right)\left\|q_{1}^{\top} \Sigma_{1}^{\top}-q_{2}^{\top} \Sigma_{2}^{\top}\right\|, \\
& \leq M \max \left(\left\|z_{i}^{\top} \underline{y}_{i}\right\|,\left\|z_{i}^{\top} \bar{y}_{i}\right\|\right)\left\|\theta_{1}-\theta_{2}\right\| .
\end{aligned}
$$

where the last equality (and the constant $M<\infty$ ) is derived from the bounds on $\Theta$.

Under conditions R.iii, we have:

$$
E\left(\max \left(\left\|z_{i}^{\top} \underline{y}_{i}\right\|,\left\|z_{i}^{\top} \bar{y}_{i}\right\|\right)\right)<\infty
$$

so that $\mathcal{F}=\left\{f_{\theta} ; \theta \in \Theta\right\}$ is a Glivenko-Cantelli class (see for instance, van der Vaart, 1998, page 271). By the definition of such a class, we have, uniformly over $\Theta$ :

$$
\frac{1}{n} \sum_{i=1}^{n} f_{\theta}\left(z_{i}, \underline{y}_{i}, \bar{y}_{i}\right)=\frac{1}{n} \sum_{i=1}^{n} z_{q i} w_{q i} \underset{n \rightarrow \infty}{\stackrel{a . s}{\rightarrow}} E\left(z_{q i} w_{q i}\right) .
$$


Also, under the conditions of Proposition 9, we have:

$$
E\left(\max \left(\left\|z_{i}^{\top} \underline{y}_{i}\right\|,\left\|z_{i}^{\top} \bar{y}_{i}\right\|\right)^{2}\right)<\infty
$$

so that $\mathcal{F}=\left\{f_{\theta} ; \theta \in \Theta\right\}$ is a Donsker class (for instance, van der Vaart, 1998, page 271). By the definition of such a class, the empirical process,

$$
\sqrt{n} \tau_{n}(q)=\sqrt{n}\left(\frac{1}{n} \sum_{i=1}^{n} z_{q i} w_{q i}-E\left(z_{q i} w_{q i}\right)\right)
$$

converges in distribution, uniformly in $\Theta$, to a Gaussian process with zero mean and covariance function:

$$
E\left(z_{q i} w_{q i} z_{r i} w_{r i}\right)-E\left(z_{q i} w_{q i}\right) E\left(z_{r i} w_{r i}\right) .
$$

The second step of the proof of Proposition 9 consists in replacing $\Sigma$ by the almost sure limit $\hat{\Sigma}_{n}$ defined above. Consistency is proved in the Additional Appendix since this result is already shown in Beresteanu and Molinari (2008). We will rely heavily on Section 19.4 of van der Vaart (1998) where relevant properties are proposed.

\section{C.1.2 Asymptotic distribution when $\Sigma$ is estimated}

We analyze the asymptotic behavior of $\tau_{n}(q)$ defined as

$$
\tau_{n}(q)=\sqrt{n}\left(\frac{1}{n} \sum_{i=1}^{n} z_{n, q i} w_{n, q i}-E\left(z_{q i} w_{q i}\right)\right)
$$

Denote $\tau_{n}(q) \equiv A_{n}(q)+B_{n}(q)$ where,

$$
A_{n}(q)=\sqrt{n}\left(\frac{1}{n} \sum_{i=1}^{n} z_{n, q i} w_{n, q i}-E\left(z_{n, q i} w_{n, q i}\right)\right), B_{n}(q)=\sqrt{n}\left(E\left(z_{n, q i} w_{n, q i}\right)-E\left(z_{q i} w_{q i}\right)\right)
$$

To begin with $A_{n}(q)$, let $\theta=(q, \Sigma)$ the true value and $\hat{\theta}_{n}=\left(q, \hat{\Sigma}_{n}\right)$ its estimate. Let us prove that if $\hat{\theta}_{n} \underset{n \rightarrow \infty}{\stackrel{P}{\rightarrow}} \theta$ uniformly in $q$ :

$$
E\left(z_{n, q i} w_{n, q i}-z_{q i} w_{q i}\right)^{2}=E\left(f_{\hat{\theta}_{n}}\left(z_{i}, \underline{y}_{i}, \bar{y}_{i}\right)-f_{\theta}\left(z_{i}, \underline{y}_{i}, \bar{y}_{i}\right)\right)^{2} \underset{n \rightarrow \infty}{\stackrel{P}{\rightarrow}} 0 .
$$

Using equation (C.11), we have:

$$
\left|f_{\hat{\theta}_{n}}\left(z_{i}, \underline{y}_{i}, \bar{y}_{i}\right)-f_{\theta}\left(z_{i}, \underline{y}_{i}, \bar{y}_{i}\right)\right| \leq M \max \left(\left\|z_{i}^{\top} \underline{y}_{i}\right\|,\left\|z_{i}^{\top} \bar{y}_{i}\right\|\right)\left\|\hat{\theta}_{n}-\theta\right\| .
$$

so that:

$$
E\left(f_{\hat{\theta}_{n}}\left(z_{i}, \underline{y}_{i}, \bar{y}_{i}\right)-f_{\theta}\left(z_{i}, \underline{y}_{i}, \bar{y}_{i}\right)\right)^{2} \leq M^{2} E\left(\max \left(\left\|z_{i}^{\top} \underline{y}_{i}\right\|,\left\|z_{i}^{\top} \bar{y}_{i}\right\|\right)^{2}\right)\left\|\hat{\theta}_{n}-\theta\right\|^{2}
$$

Under the conditions of Proposition 9, $E\left(\max \left(\left\|z_{i}^{\top} \underline{y}_{i}\right\|,\left\|z_{i}^{\top} \bar{y}_{i}\right\|\right)^{2}\right)<\infty$ and is independent of $q$. As $\left\|\hat{\theta}_{n}-\theta\right\|^{2}$ tends in distribution to 0 uniformly in $q \in \mathbb{S}$ (equation (C.9)), it tends also 
in probability to 0 , uniformly in $q \in \mathbb{S}$, which finishes the proof. Hence, we can apply Lemma 19.24 of van der Vaart (1998), so that $A_{n}(q)$ has the same distribution as:

$$
C_{n}(q)=\sqrt{n}\left(\frac{1}{n} \sum_{i=1}^{n} z_{q i} w_{q i}-E\left(z_{q i} w_{q i}\right)\right) .
$$

uniformly in $q \in S$. Therefore the problem boils down to compute the limit of processes $B_{n}(q)$ and $C_{n}(q)$ as given in the following lemma:

Lemma 13 We have, uniformly in $q \in \mathbb{S}$ :

$$
\begin{aligned}
& B_{n}(q)-\sqrt{n} E\left(\left|q^{\top}\left(\Sigma_{n}^{\top}-\Sigma^{\top}\right) z_{i}^{\top}\right|\left(\bar{y}_{i}-y_{i}\right)\left(\mathbf{1}\left\{z_{i} \Sigma q=0\right\}\right)\right) / 2 \\
& -\sqrt{n} q^{\top}\left(\hat{\Sigma}_{n}^{\top}\left(\Sigma^{\top}\right)^{-1}-I\right) \beta_{q}^{*} \underset{n \rightarrow \infty}{\stackrel{P}{\rightarrow}} 0, \\
& \text { (ii) } C_{n}(q)-\sqrt{n}\left(\frac{1}{n} \sum_{i=1}^{n} z_{q i} \varepsilon_{q i}^{*}\right)-\sqrt{n} q^{\top}\left(I-\hat{\Sigma}_{n}^{\top}\left(\Sigma^{\top}\right)^{-1}\right) \beta_{q}^{*} \underset{n \rightarrow \infty}{\stackrel{P}{\rightarrow}} 0 \text {, } \\
& \text { where } \beta_{q}^{*}=\Sigma^{\top} E\left(z_{i}^{\top} w_{q i}^{*}\right), \varepsilon_{q i}^{*}=w_{q i}-x_{i} \beta_{q}^{*} \text {, and } w_{q i}^{*}=w_{q i}+\frac{1}{2}\left(\bar{y}_{i}-\underline{y}_{i}\right) \mathbf{1}\left\{z_{q i}=0\right\} \text {. }
\end{aligned}
$$

Proof. For convenience sake, we first rewrite $w_{q i}^{*}$ :

$$
w_{q i}^{*}=\underline{y}_{i}+\frac{1}{2}\left(\bar{y}_{i}-\underline{y}_{i}\right)\left(\mathbf{1}\left\{z_{q i}>0\right\}+\mathbf{1}\left\{z_{q i} \geq 0\right\}\right) .
$$

and note that $E\left(z_{q i} w_{q i}\right)=E\left(z_{q i} w_{q i}^{*}\right)$.

We first prove (i). Write:

$$
\begin{aligned}
B_{n}(q) & =\sqrt{n}\left(E\left(z_{n, q i} w_{n, q i}\right)-E\left(z_{q i} w_{q i}^{*}\right)\right)=\sqrt{n} E\left(z_{n, q i}\left(w_{n, q i}-w_{q i}^{*}\right)\right)+E\left(\left(z_{n, q i}-z_{q i}\right) w_{q i}^{*}\right) \\
& \equiv B_{n}^{1}(q)+B_{n}^{2}(q) .
\end{aligned}
$$

By definition of $z_{n, q i}=q^{\top} \hat{\Sigma}_{n}^{\top} z_{i}^{\top}$ and $z_{q i}=q^{\top} \Sigma^{\top} z_{i}^{\top}$, the second term on the RHS is equal to:

$$
\begin{aligned}
B_{n}^{2}(q) & =\sqrt{n}\left(q^{\top}\left(\hat{\Sigma}_{n}-\Sigma\right)^{\top} E\left(z_{i}^{\top} w_{q i}^{*}\right)\right) \\
& =\sqrt{n} q^{\top}\left(\hat{\Sigma}_{n}-\Sigma\right)^{\top}\left(\Sigma^{\top}\right)^{-1} \beta_{q}^{*}, \\
& =\sqrt{n} q^{\top}\left(\hat{\Sigma}_{n}^{\top}\left(\Sigma^{\top}\right)^{-1}-I\right) \beta_{q}^{*}
\end{aligned}
$$

using the definition of $\beta_{q}^{*}$. The first term on the RHS is equal by replacement of $w_{n, q i}$ and $w_{q i}^{*}$ to:

$$
B_{n}^{1}(q)=\sqrt{n} E\left(z_{n, q i}\left(\bar{y}_{i}-\underline{y}_{i}\right)\left(\mathbf{1}\left\{z_{n, q i}>0\right\}-\frac{1}{2}\left(\mathbf{1}\left\{z_{q i}>0\right\}+\mathbf{1}\left\{z_{q i} \geq 0\right\}\right)\right)\right) .
$$

Because $z_{n, q i}$ is a root $n$ consistent estimator of $z_{q i}$, uniformly for any $z_{i}$ in a compact set, this expression converges to 0 in probability when $z_{q i} \neq 0$ since the last term, equal in this case to $\mathbf{1}\left\{z_{n, q i}>0\right\}-\mathbf{1}\left\{z_{q i}>0\right\}$ ), is identically 0 out of a (root $\mathrm{n}$ ) decreasing neighboorhood of the true value. We thus have:

$$
\sqrt{n} E\left(z_{n, q i}\left(\bar{y}_{i}-\underline{y}_{i}\right)\left(\mathbf{1}\left\{z_{n, q i}>0\right\}-\mathbf{1}\left\{z_{q i}>0\right\}\right) \mathbf{1}\left\{z_{q i} \neq 0\right\}\right)=o_{P}(1)
$$


and we are left with the term when $z_{q i}=0$ :

$$
\begin{aligned}
B_{n}^{1}(q)= & \sqrt{n} E\left(z_{n, q i}\left(\bar{y}_{i}-\underline{y}_{i}\right)\left(\mathbf{1}\left\{z_{n, q i}>0, z_{q i}=0\right\}-\frac{1}{2} \mathbf{1}\left\{z_{q i}=0\right\}\right)\right)+o_{P}(1) \\
= & \sqrt{n} E\left(\frac{z_{n, q i}}{2}\left(\bar{y}_{i}-\underline{y}_{i}\right) \mathbf{1}\left\{z_{n, q i}>0, z_{q i}=0\right\}\right) \\
& -\sqrt{n} E\left(\frac{z_{n, q i}}{2}\left(\bar{y}_{i}-\underline{y}_{i}\right) \mathbf{1}\left\{z_{n, q i} \leq 0, z_{q i}=0\right\}\right)+o_{P}(1) \\
= & \sqrt{n} E\left(\frac{\left|z_{n, q i}\right|}{2}\left(\bar{y}_{i}-\underline{y}_{i}\right) \mathbf{1}\left\{z_{q i}=0\right\}\right)+o_{P}(1) \\
= & \sqrt{n} E\left(\frac{\left|z_{n, q i}-z_{q i}\right|}{2}\left(\bar{y}_{i}-\underline{y}_{i}\right) \mathbf{1}\left\{z_{q i}=0\right\}\right)+o_{P}(1) \\
= & \sqrt{n} E\left(\left|q^{\top}\left(\Sigma_{n}^{\top}-\Sigma^{\top}\right) z_{i}^{\top}\right|\left(\bar{y}_{i}-\underline{y}_{i}\right) \mathbf{1}\left\{z_{q i}=0\right\}\right) / 2+o_{P}(1) .
\end{aligned}
$$

Adding $B_{n}^{2}(q)$ and $B_{n}^{1}(q)$ finishes the proof of (i).

To prove (ii), use $z_{q}=q^{\top} \Sigma^{\top} z_{i}^{\top}$ to write :

$$
C_{n}(q)=\sqrt{n}\left(\frac{1}{n} \sum_{i=1}^{n} z_{q i} w_{q i}-E\left(q^{\top} \Sigma^{\top} z_{i}^{\top} w_{q i}^{*}\right)\right) .
$$

Using $w_{q i}=x_{i} \beta_{q}^{*}+\varepsilon_{q i}^{*}$, we have:

$$
C_{n}(q)=\sqrt{n}\left(\frac{1}{n} \sum_{i=1}^{n} z_{q i} \varepsilon_{q i}^{*}\right)+\sqrt{n}\left(\frac{1}{n} \sum_{i=1}^{n} q^{\top} \Sigma^{\top} z_{i}^{\top} x_{i} \beta_{q}^{*}-E\left(q^{\top} \Sigma^{\top} z_{i}^{\top} w_{q i}^{*}\right)\right)
$$

Using $E\left(z_{q i} w_{q i}^{*}\right)=E\left(z_{q i} w_{q i}\right)=E\left(z_{q i} x_{i} \beta_{q}^{*}\right)$, the second term on the right hand side is equal to:

$$
\begin{aligned}
\sqrt{n} q^{\top} \Sigma^{\top} & \left(\frac{1}{n} \sum_{i=1}^{n} z_{i}^{\top} x_{i}\right) \beta_{q}^{*}-\sqrt{n} q^{\top} \beta_{q}^{*} \\
& =\sqrt{n} q^{\top}\left(\Sigma^{\top}\left(\hat{\Sigma}_{n}^{u \top}\right)^{-1}-I\right) \beta_{q}^{*} \\
& =\sqrt{n} q^{\top}\left(\Sigma^{\top}\left(\hat{\Sigma}_{n}^{\top}\right)^{-1}-I\right) \beta_{q}^{*}+o_{p}(1) \\
& =\sqrt{n} q^{\top} \Sigma^{\top}\left(\hat{\Sigma}_{n}^{\top}\right)^{-1}\left(I-\hat{\Sigma}_{n}^{\top}\left(\Sigma^{\top}\right)^{-1}\right) \beta_{q}^{*}+o_{p}(1)
\end{aligned}
$$

The third line uses that $\sqrt{n}\left(\hat{\Sigma}_{n}^{u}-\hat{\Sigma}_{n}\right) \underset{n \rightarrow \infty}{\stackrel{P}{\rightarrow}} 0$ by equation (C.9) and uniform bounds on $q, \Sigma$ and $\beta_{q}^{*}$. Moreover, as $\hat{\Sigma}_{n}$ is bounded and its inverse exists, $\Sigma^{\top}\left(\hat{\Sigma}_{n}^{\top}\right)^{-1} \underset{n \rightarrow \infty}{\stackrel{a . s}{\rightarrow}} I$, and we have, uniformly in $q$ :

$$
C_{n}(q)=\sqrt{n}\left(\frac{1}{n} \sum_{i=1}^{n} q^{\top} \Sigma^{\top} z_{i}^{\top} \varepsilon_{q i}^{*}\right)+\sqrt{n} q^{\top}\left(I-\Sigma^{-1} \hat{\Sigma}_{n}\right)^{\top} \beta_{q}^{*}+o_{p}(1) .
$$

Summing the different terms in the Lemma implies that $\tau_{n}(q)$ is asymptotically equivalent to:

$$
\sqrt{n}\left(\frac{1}{n} \sum_{i=1}^{n} z_{q i} \varepsilon_{q i}^{*}\right)+\sqrt{n} E\left(\left|q^{\top}\left(\Sigma_{n}^{\top}-\Sigma^{\top}\right) z_{i}^{\top}\right|\left(\bar{y}_{i}-\underline{y}_{i}\right) \mathbf{1}\left\{z_{q i}=0\right\}\right) / 2
$$


If there are no exposed faces (i.e., $\operatorname{Pr}\left(z_{i} \Sigma q=0\right)=0$ ), the second term is identically equal to zero whereas $\varepsilon_{q i}^{*}$ boils down to the residual of the IV regression of $w_{q}$ on to $x$ using instruments $z$ so that $\tau_{n}(q)$ converges in distribution, uniformly in $q$, to a Gaussian process centered at zero and of covariance function:

$$
E\left(z_{q i} \varepsilon_{q i} \varepsilon_{r i} z_{r i}\right)
$$

with $\varepsilon_{q i}=w_{q i}-x_{i} \beta_{q}$.

Suppose that there exist exposed faces $\left(\operatorname{Pr}\left(z_{q i}=0\right)>0\right)$. Write:

$$
\Sigma q=\left(I_{p} \otimes q^{\top}\right) \operatorname{vec}\left(\Sigma^{\top}\right)
$$

so that, using the asymptotic normality of the estimate of $\operatorname{vec}\left(\Sigma^{\top}\right)$ in equation (C.10) we have:

$\sqrt{n} q^{\top}\left(\Sigma_{n}^{\top}-\Sigma^{\top}\right) z_{i}^{\top}=\sqrt{n}\left(\operatorname{vec}\left(\Sigma_{n}^{\top}\right)^{\top}-\operatorname{vec}\left(\Sigma^{\top}\right)^{\top}\right)\left(I_{p} \otimes q\right) z_{i}^{\top}=\sqrt{n} \eta^{\top} W^{1 / 2}\left(I_{p} \otimes q\right) z_{i}^{\top}+o_{P}(1)$,

where $\eta$ is a multivariate standard normal random variable of dimension $p^{2}$ independent of $z_{i}$.

\section{C.2 Proof of Proposition 10}

When $\beta_{0}$ is outside (resp. inside) set $B$ but not on the frontier, we know that $\inf _{q} T_{\infty}(q)$ is strictly negative (resp. positive). As $T_{n}(q)$ converges uniformly in $q$ to $T_{\infty}(q), \min _{q} T_{n}(q)$ is negative (resp. positive) and bounded away from zero for $n$ sufficiently large. $\sqrt{n} T_{n}\left(q_{n}\right)$ tends therefore to $-\infty$ (resp. $+\infty)$.

Consider now the case $\beta_{0} \in \partial B$ and let $\mathcal{Q}\left(\beta_{0}\right)$ the set of all $q_{0} \in \mathbb{S}$ which minimize $T_{\infty}\left(q ; \beta_{0}\right)$, i.e., the set of all $q_{0} \in \mathbb{S}$ satisfying $\delta^{*}\left(q_{0} \mid B\right)=q_{0}^{\top} \beta_{0} . \mathcal{Q}\left(\beta_{0}\right)$ is a non-empty compact subset of $\mathbb{S}$. We first consider the case in which $\mathcal{Q}\left(\beta_{0}\right)$ is a singleton. In the second part, the proof is extended to the case in which $\mathcal{Q}\left(\beta_{0}\right)$ may contain more than one element of $\mathbb{S}$.

\section{C.2.1 $\mathcal{Q}\left(\beta_{0}\right)$ is a singleton: $\mathcal{Q}\left(\beta_{0}\right)=\left\{q_{0}\right\}$}

As $\delta^{*}(q \mid B)$ is differentiable (assumption D), the empirical stochastic process defined for $q \in \mathbb{S}$ as,

$$
\sqrt{n}\left(T_{n}\left(q ; \beta_{0}\right)-T_{\infty}\left(q ; \beta_{0}\right)\right)=\sqrt{n}\left(\hat{\delta}_{n}^{*}(q \mid B)-\delta^{*}(q \mid B)\right)=\tau_{n}(q)
$$

converges to a Gaussian process (Proposition 9) whose sample paths are uniformly continuous on the unit sphere $\mathbb{S}$ endowed with the usual Euclidean norm. Hence $\tau_{n}($.$) is stochastically$ equicontinuous (for instance, p. 2251 of Andrews, 1994).

Let $q_{n} \in \mathbb{S}$ be any sequence of directions defined as near minimizers of the empirical counterpart $T_{n}\left(q ; \beta_{0}\right)$ defined as,

$$
T_{n}\left(q_{n} ; \beta_{0}\right) \leq \min _{q} T_{n}\left(q ; \beta_{0}\right)+o_{P}(1) .
$$

Standard arguments employed for Z-estimators (e.g. van der Vaart, 1998) when the objective function has a unique well separated minimum, imply that:

$$
\operatorname{plim}_{n \rightarrow \infty} q_{n}=q_{0} .
$$

Because (i) $\tau_{n}($.$) is stochastically equicontinuous (ii) q_{n} \in \mathbb{S}$ (iii) $\operatorname{plim}_{n \rightarrow \infty} q_{n}=q_{0}$, Andrews (1994, equation (3.36), p. 2265) shows that:

$$
\sqrt{n}\left(T_{n}\left(q_{n} ; \beta_{0}\right)-T_{n}\left(q_{0} ; \beta_{0}\right)\right) \underset{n \rightarrow \infty}{\stackrel{P}{\rightarrow}} 0 .
$$

The proof finishes by using the asymptotic distribution of $\sqrt{n} T_{n}\left(q_{0} ; \beta_{0}\right)$ as stated in the text. 


\section{C.2.2 $\mathcal{Q}\left(\beta_{0}\right)$ is not a singleton}

The proof proceeds in various steps:

1. We select and characterize a unique $q_{0}^{*}$ from $\mathcal{Q}\left(\beta_{0}\right)$.

2. We construct a sequence of well separated minima of minimization programs which tends to $q_{0}^{*}$.

3. We show that any sequence of minimizers of the empirical programs converge to $q_{0}^{*}$.

1. The selection of a single $q_{0}^{*} \in \mathcal{Q}\left(\beta_{0}\right) \quad$ For this, we select a vector oriented outwards $B$ and consider its projection on the smallest convex cone which includes $\mathcal{Q}\left(\beta_{0}\right)$ :

$$
\mathcal{C}\left(\beta_{0}\right)=\left\{\lambda q_{0} ; q_{0} \in \mathcal{Q}\left(\beta_{0}\right), \lambda \geq 0\right\}=\left\{v ; \delta^{*}(v \mid B)-v^{\top} \beta_{0} \leq 0\right\} .
$$

The vector oriented outwards $B$ can be constructed as the difference between $\beta_{0}$ which is a frontier point of $B$ and any interior point $\beta^{*}$ of $B$. For instance the "center" of $B$ obtained by setting $\left.u(z)=\frac{\Delta(z)+\bar{\Delta}(z)}{2}\right)$ is interior and

$$
\beta^{*}=E\left(\Sigma^{\top} z^{\top} \frac{\bar{y}+\underline{y}}{2}\right)
$$

Denote $v_{0}=\beta_{0}-\beta^{*} \neq 0$ and note that, as $\beta^{*} \in \operatorname{int}(B)$, we have for all $q_{0}$ in $\mathcal{Q}\left(\beta_{0}\right)$ :

$$
\delta^{*}\left(q_{0} \mid B\right)-q_{0}^{\top} \beta^{*}>0 \Longrightarrow q_{0}^{\top} v_{0}>0 .
$$

The projection of $v_{0}$ on the convex cone $\mathcal{C}\left(\beta_{0}\right)$ is given by:

$$
\min _{v, \delta^{*}(v \mid B)-v^{\top} \beta_{0} \leq 0} \frac{\left(v_{0}-v\right)^{\top}\left(v_{0}-v\right)}{2}
$$

This projection is unique and defined by $v_{0}^{*}=\lambda^{*} q_{0}^{*}$ where $\left(\lambda^{*}, q_{0}^{*}\right)$ is the argument of the minimum:

$$
\min _{\left(\lambda \geq 0, q \in \mathcal{Q}\left(\beta_{0}\right)\right)}\left(v_{0}-\lambda q\right)^{\top}\left(v_{0}-\lambda q\right) \propto \min _{\left(\lambda \geq 0, q \in \mathcal{Q}\left(\beta_{0}\right)\right)}\left\{-2 \lambda q^{\top} v_{0}+\lambda^{2}\right\}
$$

which yields $\lambda^{*}=q_{0}^{* \top} v_{0}>0$ (see equation C.14) whereas $q_{0}^{*}$ is the argument of:

$$
\max _{q \in Q\left(\beta_{0}\right)} q^{\top} v_{0}
$$

Vector $q_{0}^{*}$ is unique because it is a (normalized) projection. Furthermore, when $\frac{v_{0}}{\left\|v_{0}\right\|} \in \mathcal{Q}\left(\beta_{0}\right)$ (or equivalently $v_{0} \in \mathcal{C}\left(\beta_{0}\right)$ ), we have $q_{0}^{*}=\frac{v_{0}}{\left\|v_{0}\right\|}$ whereas in other cases $q_{0}^{*}$ belongs to the frontier of $\mathcal{Q}\left(\beta_{0}\right)$.

2. Minimization programs whose well separated solutions converges to $q_{0}^{*} \quad$ The estimation of $q_{0}^{*}$ cannot proceed directly from program (C.15) since we do not know the set of constraints, $\mathcal{Q}\left(\beta_{0}\right)$. Consider the generalization of (C.15) for any $\alpha \geq 0$ :

$$
b(\alpha) \equiv \min _{v, \delta^{*}(v \mid B)-v^{\top} \beta_{0} \leq \alpha} \frac{\left(v_{0}-v\right)^{\top}\left(v_{0}-v\right)}{2}
$$


where $b(\alpha)$ is continuous and non increasing in $\alpha$ because the constraint is continuous. The unique solution of this program, denoted $v_{\alpha}^{*}$, is the projection of $v_{0}=\beta_{0}-\beta^{*}$ on the convex cone $\left\{v \in \mathbb{R}^{p}, \delta^{*}(v \mid B)-v^{\top} \beta_{0} \leq \alpha\right\}$.

We state a sequence of Lemmas that are proved below in Section C.2.3.

It turns out that the following equivalent characterization to this program will be more amenable to estimation.

Lemma 14 For any $\alpha>0$, the strictly convex program (C.16) is equivalent to the minimization of:

$$
\Psi_{a}(q)=\delta^{*}(q \mid B)-q^{\top} \beta_{0}-a q^{\top} v_{0},
$$

where $a$ is an increasing function of $\alpha$.

This equivalence covers the case where $a>0$. We need to complete this result by showing how the minimizer $q_{a}$ of $\Psi_{a}(q)$ converges to $q_{0}^{*}$ when $a \rightarrow 0$.

Lemma 15 The limit of the sequence $\left\{q_{a}\right\}_{a>0}$ exists when $a \rightarrow 0$ and is equal to $q_{0}^{*}$. Furthermore:

$$
\Psi_{a}\left(q_{0}^{*}\right)-\Psi_{a}\left(q_{a}\right)=o(a)
$$

Moreover, we have the following uniform result:

\section{Lemma 16}

$$
\forall \varepsilon>0, \exists a_{0}>0, \exists \eta>0 \text { such that } \inf _{0<a \leq a_{0},\left\|q-q_{0}^{*}\right\| \geq \varepsilon} \frac{\Psi_{a}(q)-\Psi_{a}\left(q_{a}\right)}{a}>\eta .
$$

3. Estimation of $q_{a}$ and convergence to $q_{0}^{*}$ Finally, we construct the estimate of $q_{a}$. Fix $a>0$. Define the perturbed estimated convex program as:

$$
\Psi_{n, a}\left(q ; \beta_{0}\right)=\hat{\delta}_{n}^{*}(q \mid B)-q^{\top} \beta_{0}-a q^{\top} v_{0, n}
$$

where $v_{0, n}=\beta_{0}-\hat{\beta}_{n}^{*}$ and $\hat{\beta}_{n}^{*}=\frac{1}{n} \sum_{i=1}^{n} \hat{\Sigma}_{n}^{\top} z_{i} \frac{\bar{y}_{i}+\underline{y}_{i}}{2}$.

Define $q_{n, a}$ as a near minimizer of $\Psi_{n, a}$ :

$$
\Psi_{n, a}\left(q_{n, a}\right) \leq \inf _{q} \Psi_{n, a}(q)+O_{P}\left(n^{-1 / 2}\right) .
$$

We have

$$
\Psi_{n, a}\left(q_{n, a}\right) \leq \Psi_{n, a}\left(q_{a}\right)+O_{P}\left(n^{-1 / 2}\right) .
$$

whereas the square-root uniform convergence of $\Psi_{n, a}$ to $\Psi_{a}$ ensures that:

$$
\Psi_{n, a}\left(q_{n, a}\right)=\Psi_{a}\left(q_{n, a}\right)+O_{P}\left(n^{-1 / 2}\right) .
$$

Using successively the last equality and the previous inequality, we canwrite,

$$
\begin{aligned}
0 \leq \Psi_{a}\left(q_{n, a}\right)-\Psi_{a}\left(q_{a}\right) & =\Psi_{n, a}\left(q_{n, a}\right)-\Psi_{a}\left(q_{a}\right)+O_{P}\left(n^{-1 / 2}\right), \\
& \leq \Psi_{n, a}\left(q_{a}\right)-\Psi_{a}\left(q_{a}\right)+O_{P}\left(n^{-1 / 2}\right), \\
& \leq \sup _{q}\left|\Psi_{a}(q)-\Psi_{n, a}(q)\right|+O_{P}\left(n^{-1 / 2}\right) .
\end{aligned}
$$


We thus have:

$$
\frac{\Psi_{a}\left(q_{n, a}\right)-\Psi_{a}\left(q_{a}\right)}{a} \leq \frac{\sup _{q}\left|\Psi_{a}(q)-\Psi_{n, a}(q)\right|+O_{P}\left(n^{-1 / 2}\right)}{a} .
$$

Let $a_{n}=O\left(n^{-\alpha}\right)$ a sequence such that $\alpha<1 / 2$. Because of equicontinuity and $n^{1 / 2}$ convergence of $\hat{\delta}_{n}^{*}(q \mid B)$ to $\delta^{*}(q \mid B)$ and of $v_{0, n}$ to $v_{0}$, we have that:

$$
n^{\alpha} \sup _{q}\left|\Psi_{a_{n}}(q)-\Psi_{n, a_{n}}(q)\right| \underset{n \rightarrow \infty}{\stackrel{P}{\rightarrow}} 0
$$

Then:

$$
\frac{\Psi_{a_{n}}\left(q_{n, a_{n}}\right)-\Psi_{a_{n}}\left(q_{a_{n}}\right)}{a_{n}} \leq o_{P}(1)
$$

and therefore:

$$
\forall \eta>0, \lim _{n \rightarrow \infty} \operatorname{Pr}\left(\frac{\Psi_{a_{n}}\left(q_{n, a_{n}}\right)-\Psi_{a_{n}}\left(q_{a_{n}}\right)}{a_{n}}>\eta\right)=0
$$

By condition (C.17), for any $\varepsilon>0$, there exist $n_{0}$ and $\eta>0$ such that, for $n \geq n_{0}$ the event:

$$
\left\{d\left(q_{n, a_{n}}, q_{0}^{*}\right) \geq \varepsilon\right\} \subset\left\{\frac{\Psi_{a_{n}}\left(q_{n, a_{n}}\right)-\Psi_{a_{n}}\left(q_{a_{n}}\right)}{a_{n}}>\eta\right\} .
$$

Therefore:

$$
\forall \varepsilon>0, \lim _{n \rightarrow \infty} \operatorname{Pr}\left(d\left(q_{n, a_{n}}, q_{0}^{*}\right) \geq \varepsilon\right)=0 \Longrightarrow q_{n, a_{n}}-q_{0}^{*} \underset{n \rightarrow \infty}{\stackrel{P}{\rightarrow}} 0 .
$$

To finish the proof of Proposition 10 we can now use the same argument than in Section C.2.1 so that:

$$
\sqrt{n}\left(T_{n}\left(q_{n, a_{n}} ; \beta_{0}\right)-T_{n}\left(q_{0}^{*} ; \beta_{0}\right)\right) \underset{n \rightarrow \infty}{\stackrel{P}{\rightarrow}} 0 .
$$

The variance of $T_{n}\left(q_{0}^{*} ; \beta_{0}\right)$ is estimated as the variance of $T_{n}\left(q_{n, a_{n}} ; \beta_{0}\right)$.

\section{C.2.3 Proofs of Lemma 14 to 16}

Proof of Lemma 14: Let $\alpha_{0}=\delta^{*}\left(v_{0} \mid B\right)-v_{0}^{\top} \beta_{0}$, we have $v_{\alpha}^{*}=v_{0}$ for any $\alpha \geq \alpha_{0}$ whereas in other cases the optimal solution $v_{\alpha}^{*}$ is such that the constraint is binding, $\delta^{*}\left(v_{\alpha}^{*} \mid B\right)-v_{\alpha}^{* \top} \beta_{0}=\alpha$. If $\frac{v_{0}}{\left\|v_{0}\right\|} \in \mathcal{Q}\left(\beta_{0}\right)$, we have that $\alpha_{0}=0$ and

$$
q_{0}^{*}=\frac{v_{\alpha}^{*}}{\left\|v_{\alpha}^{*}\right\|}=\frac{v_{0}}{\left\|v_{0}\right\|}, \forall \alpha \geq 0
$$

When $\frac{v_{0}}{\left\|v_{0}\right\|} \notin \mathcal{Q}\left(\beta_{0}\right)$ and $\alpha$ runs from 0 to $\alpha_{0}$, $v_{\alpha}^{*}$ describes a trajectory between $v_{0}^{*}$ and $v_{0}$. We now characterize this trajectory.

It is easier to work with the equivalent dual program (Rockafellar, 1970):

$$
\alpha=\min _{v, \frac{\left(v_{0}-v\right)^{\top}\left(v_{0}-v\right)}{2} \leq b(\alpha)}\left(\delta^{*}(v \mid B)-v^{\top} \beta_{0}\right),
$$

where $b(\alpha)$ runs from $\frac{\left(v_{0}-v_{0}^{*}\right)^{\top}\left(v_{0}-v_{0}^{*}\right)}{2}$ to 0 to generate the same trajectory $\left\{v_{\alpha}^{*}\right\}_{\alpha \geq 0}$. Writing the program (C.18) as the Lagrangian where $a>0$ :

$$
L(v, a)=\delta^{*}(v \mid B)-v^{\top} \beta_{0}+a\left(\frac{\left(v_{0}-v\right)^{\top}\left(v_{0}-v\right)}{2}-b(\alpha)\right)
$$


we obtain the first order condition (by assumption $\mathrm{D}, \delta^{*}(v \mid B)$ is differentiable):

$$
\beta_{q_{\alpha}}-\beta_{0}-a(\alpha)\left(v_{0}-v_{\alpha}^{*}\right)=0
$$

where $q_{\alpha}=\frac{v_{\alpha}^{*}}{\left\|v_{\alpha}^{*}\right\|} \in \mathbb{S}$ and $\beta_{q_{\alpha}}=\left.\frac{\partial \delta^{*}(v \mid B)}{\partial v}\right|_{v_{\alpha}^{*}}$. To obtain $a$, multiply the equation by $\left(v_{0}-v_{\alpha}^{*}\right)^{\top}$ :

$$
2 a(\alpha) b(\alpha)=\left(v_{0}-v_{\alpha}^{*}\right)^{\top}\left(\beta_{q_{\alpha}}-\beta_{0}\right) .
$$

When $\alpha=0, \beta_{q_{\alpha}}=\beta_{0}$ and therefore $a(\alpha)=0$ since $\frac{v_{0}}{\left\|v_{0}\right\|} \notin \mathcal{Q}\left(\beta_{0}\right)$ and $b(\alpha)>0$. Furthermore, $a(\alpha)$ is continuous in $\alpha$ for any $\alpha<\alpha_{0}$ since all objects in the expression are continuous.

We now prove that $a(\alpha)$ is increasing with $\alpha$. Consider $0<\alpha<\alpha^{\prime}<\alpha_{0}$ and the optimal solutions $v_{\alpha}^{*}$ and $v_{\alpha^{\prime}}^{*}$, where $v_{\alpha}^{*} \neq v_{\alpha^{\prime}}^{*}$ because:

$$
\delta^{*}\left(v_{\alpha}^{*} \mid B\right)-v_{\alpha}^{* \top} \beta_{0}=\alpha<\delta^{*}\left(v_{\alpha^{\prime}}^{*} \mid B\right)-v_{\alpha^{\prime}}^{* \top} \beta_{0}=\alpha^{\prime} .
$$

Note that by optimality:

$$
\begin{gathered}
L\left(v_{\alpha}^{*}, a(\alpha)\right)=\delta^{*}\left(v_{\alpha}^{*} \mid B\right)-v_{\alpha}^{* \top} \beta_{0}<\delta^{*}\left(v_{\alpha^{\prime}}^{*} \mid B\right)-v_{\alpha^{\prime}}^{* \top} \beta_{0}+a(\alpha)\left(\frac{\left(v_{0}-v_{\alpha^{\prime}}^{*}\right)^{\top}\left(v_{0}-v_{\alpha^{\prime}}^{*}\right)}{2}-b(\alpha)\right), \\
L\left(v_{\alpha}^{*}, a\left(\alpha^{\prime}\right)\right)=\delta^{*}\left(v_{\alpha}^{*} \mid B\right)-v_{\alpha}^{* \top} \beta_{0}+a\left(\alpha^{\prime}\right)\left(\frac{\left(v_{0}-v_{\alpha}^{*}\right)^{\top}\left(v_{0}-v_{\alpha}^{*}\right)}{2}-b\left(\alpha^{\prime}\right)\right)>\delta^{*}\left(v_{\alpha^{\prime}}^{*} \mid B\right)-v_{\alpha^{\prime}}^{* \top} \beta_{0} .
\end{gathered}
$$

so that by differencing:

$$
a\left(\alpha^{\prime}\right)\left(b(\alpha)-b\left(\alpha^{\prime}\right)\right)>-a(\alpha)\left(b\left(\alpha^{\prime}\right)-b(\alpha)\right) \Rightarrow\left(a\left(\alpha^{\prime}\right)-a(\alpha)\right)\left(b(\alpha)-b\left(\alpha^{\prime}\right)\right)>0 .
$$

As $b(\alpha)$ is non increasing, it implies that $a(\alpha)$ is increasing with $\alpha$ from $a(0)=0$ to $\lim _{\alpha \rightarrow \alpha_{0}} a(\alpha)=$ $+\infty$.

We can thus generates the $\operatorname{arc}\left\{v_{\alpha}^{*}\right\}_{\alpha>0}$ equivalently by making $a$ varies between 0 and $\infty$. Let us rewrite the minimization program (C.19) in order to consider vectors on $\mathbb{S}$ since estimates are defined on $\mathbb{S}$ only:

$$
\begin{aligned}
L(\lambda q, a) & =\delta^{*}(\lambda q \mid B)-(\lambda q)^{\top} \beta_{0}+a\left(\frac{\left(v_{0}-\lambda q\right)^{\top}\left(v_{0}-\lambda q\right)}{2}-b(\alpha)\right), \\
& =\lambda\left(\delta^{*}(q \mid B)-q^{\top} \beta_{0}\right)+a\left(\frac{\left(v_{0}-\lambda q\right)^{\top}\left(v_{0}-\lambda q\right)}{2}-b(\alpha)\right) .
\end{aligned}
$$

Minimizing wrt $\lambda$ yields the FOC for the optimal solution $\lambda_{q}$ :

$$
\delta^{*}(q \mid B)-q^{\top} \beta_{0}+a\left(\lambda_{q}-q^{\top} v_{0}\right)=0,
$$

which implies that:

$$
\begin{aligned}
L\left(\lambda_{q} q, a\right) & =a\left(-\frac{\lambda_{q}^{2}}{2}-b(\alpha)\right), \\
-a \lambda_{q} & =\delta^{*}(q \mid B)-q^{\top} \beta_{0}-a q^{\top} v_{0} \equiv \Psi_{a}(q) .
\end{aligned}
$$

When $a>0$, minimizing $L\left(\lambda_{q} q, a\right)$ is equivalent to maximizing $\lambda_{q}$ and thus equivalent to minimizing $\Psi_{a}(q)$. As $L\left(\lambda_{q} q, a\right)$ is a strictly convex program, the minimizer of $\Psi_{a}(q)$ is unique and well separated. 
Proof of Lemma 15: To begin with, it is useful to note that $-a\left\|v_{0}\right\|$ provides a lower bound of $\Psi_{a}(q)$,

$$
\Psi_{a}(q)=\delta^{*}(q \mid B)-q^{\top} \beta_{0}-a q^{\top} v_{0} \geq-a\left\|v_{0}\right\|,
$$

because $\beta_{0} \in B$ and $q$ and $\frac{v_{0}}{\left\|v_{0}\right\|}$ belong to $\mathbb{S}$.

We are going to consider in turn two cases:

- Assume first that $\frac{v_{0}}{\left\|v_{0}\right\|} \in \mathcal{Q}\left(\beta_{0}\right)$. In such a case, $q_{0}^{*}=\frac{v_{0}}{\left\|v_{0}\right\|}$ and $\Psi_{a}\left(q_{0}^{*}\right)=-a\left\|v_{0}\right\|$. Hence, given that $q_{a}$ is unique and that $-a\left\|v_{0}\right\|$ is a lower bound for $\Psi_{a}(q)$, we have necessarily $q_{a}=q_{0}^{*}$ for any $a>0$.

- Assume now that $\frac{v_{0}}{\left\|v_{0}\right\|} \notin \mathcal{Q}\left(\beta_{0}\right)$. By definition of $q_{a}$ as a minimum,

$$
\Psi_{a}\left(q_{a}\right)=\delta^{*}\left(q_{a} \mid B\right)-q_{a}^{\top} \beta_{0}-a q_{a}^{\top} v_{0} \leq \Psi_{a}\left(q_{0}^{*}\right)=-a q_{0}^{* \top} v_{0},
$$

since $\delta^{*}\left(q_{0}^{*} \mid B\right)=q_{0}^{* \top} \beta_{0}$. It implies that:

$$
0 \leq \delta^{*}\left(q_{a} \mid B\right)-q_{a}^{\top} \beta_{0} \leq a\left(q_{a}-q_{0}^{*}\right)^{\top} v_{0} \leq 2 a\left\|v_{0}\right\|
$$

since $\beta_{0} \in B$, (the left-hand side, $\delta^{*}\left(q_{a} \mid B\right)-q_{a}^{\top} \beta_{0}$, is non-negative) and since $\left\|q_{a}-q_{0}^{*}\right\| \leq$ 2. Consequently, we have,

$$
\lim _{a \rightarrow 0}\left(\delta^{*}\left(q_{a} \mid B\right)-q_{a}^{\top} \beta_{0}\right)=0,
$$

and the distance between set $\mathcal{Q}\left(\beta_{0}\right)$ and $q_{a}$ tends to zero by continuity of the function $\delta^{*}(q \mid B)-q^{\top} \beta_{0}$.

Consider now $q_{m}$ any accumulation point of the sequence $q_{a}$ i.e., any point satisfying, $\forall \eta>0, \exists a_{0}>0$ such that $\forall a<a_{0},\left\|q_{a}-q_{m}\right\|<\eta .{ }^{16}$ Because $\mathcal{Q}\left(\beta_{0}\right)$ is compact, $q_{m} \in \mathcal{Q}\left(\beta_{0}\right)$. We are going to show that $q_{m}=q_{0}^{*}$. By definition of $q_{a}$ and $q_{0}^{*}$, we have

$$
\frac{\Psi_{a}\left(q_{a}\right)}{a} \leq \frac{\Psi_{a}\left(q_{0}^{*}\right)}{a}=-q_{0}^{* \top} v_{0} \leq-q_{m}^{\top} v_{0}
$$

where the first inequality holds true because $q_{a}$ minimizes $\Psi_{a}$ on the unit sphere whereas the second inequality holds true because $q_{m} \in \mathcal{Q}\left(\beta_{0}\right)$ and $q_{0}^{*}$ maximizes $q^{\top} v_{0}$ on $\mathcal{Q}\left(\beta_{0}\right)$. Furthermore, since $\delta^{*}(q \mid B) \geq q^{\top} \beta_{0}$ for any $q$ on the unit sphere, we have,

$$
\frac{\Psi_{a}\left(q_{a}\right)}{a}=\frac{\delta^{*}\left(q_{a} \mid B\right)-q_{a}^{\top} \beta_{0}}{a}-q_{a}^{\top} v_{0} \geq-q_{a}^{\top} v_{0}
$$

Combining this inequality with the two previous ones, we have,

$$
-q_{a}^{\top} v_{0} \leq-q_{0}^{* \top} v_{0} \leq-q_{m}^{\top} v_{0}
$$

By taking limits and using that $q_{a}$ tends to $q_{m}$ when $a$ tends to zero, we obtain that $q_{m}^{\top} v_{0}=$ $q_{0}^{* \top} v_{0}$. Given the definition of $q_{0}^{*}$, it means that $q_{m}$ is the argument of $\max _{q \in Q\left(\beta_{0}\right)} q^{\top} v_{0}$. But this argument is unique and is precisely $q_{0}^{*}$. Hence, we have necessarily $q_{m}=q_{0}^{*}$ and therefore:

$$
\lim _{a \rightarrow 0}\left\|q_{a}-q_{0}^{*}\right\|=0
$$

Furthermore, as:

$$
0 \leq \frac{\Psi_{a}\left(q_{0}^{*}\right)-\Psi_{a}\left(q_{a}\right)}{a} \leq\left(q_{a}-q_{0}^{*}\right)^{\top} v_{0}
$$

we have:

$$
\Psi_{a}\left(q_{0}^{*}\right)-\Psi_{a}\left(q_{a}\right)=o(a) .
$$

\footnotetext{
${ }^{16}$ Such a sequence exists as the distance between $q_{a}$ and $\mathcal{Q}\left(\beta_{0}\right)$, a compact set, tends to zero. In the following we will work with $a$ instead of working with a sequence indexed by $a$ without loss of generality.
} 
Proof of Lemma 16: First, the Lemma is trivially satisfied when $\frac{v_{0}}{\left\|v_{0}\right\|} \in \mathcal{Q}\left(\beta_{0}\right)$ since $q_{a}=$ $q_{0}^{*}=\frac{v_{0}}{\left\|v_{0}\right\|}$ and therefore:

$$
\frac{\Psi_{a}(q)-\Psi_{a}\left(q_{a}\right)}{a} \geq-\left(q-\frac{v_{0}}{\left\|v_{0}\right\|}\right)^{\top} v_{0}=\frac{1}{2}\left\|v_{0}\right\|\left\|q-\frac{v_{0}}{\left\|v_{0}\right\|}\right\|^{2},
$$

the last equality resulting from the following expansion:

$$
\|q\|^{2}=1=\left\|q-\frac{v_{0}}{\left\|v_{0}\right\|}+\frac{v_{0}}{\left\|v_{0}\right\|}\right\|^{2}=1+2\left(q-\frac{v_{0}}{\left\|v_{0}\right\|}\right)^{\top} \frac{v_{0}}{\left\|v_{0}\right\|}+\left\|q-\frac{v_{0}}{\left\|v_{0}\right\|}\right\|^{2} .
$$

Consequently, this quantity is bounded from below by a positive number when $\left\|q-\frac{v_{0}}{\left\|v_{0}\right\|}\right\| \geq \varepsilon$.

Assume now that $\frac{v_{0}}{\left\|v_{0}\right\|} \notin \mathcal{Q}\left(\beta_{0}\right)$. We will first show that, for a given $q$, the infimum if it is attained when $a$ tends to zero is strictly positive. Using the results of Lemma (15), we know that when $a \rightarrow 0, q_{a} \rightarrow q_{0}^{*}$ and $\frac{\Psi_{a}\left(q_{a}\right)}{a} \rightarrow-q_{0}^{* \top} v_{0}$.

- Either $q \in \mathcal{Q}\left(\beta_{0}\right)$ and $\frac{\Psi_{a}(q)}{a}=-q^{\top} v_{0} \geq-q_{0}^{* \top} v_{0}$, by construction of $q_{0}^{*}$. Consequently,

$$
\frac{\Psi_{a}(q)-\Psi_{a}\left(q_{a}\right)}{a} \underset{a \rightarrow 0}{\rightarrow}-\left(q-q_{0}^{*}\right)^{\top} v_{0}
$$

which is strictly positive when $\left\|q-q_{0}^{*}\right\| \geq \varepsilon$.

- Or $q \notin \mathcal{Q}\left(\beta_{0}\right)$. In this case $\frac{\Psi_{a}(q)}{a} \rightarrow+\infty$ and cannot deliver the infimum.

As $q_{a}$ tends to $q_{0}^{*}$ when $a$ tends to zero, there exists some $a_{0}$ for which the joint events $\left\{0<a \leq a_{0}\right\}$ and $\left\{\left\|q-q_{0}^{*}\right\| \geq \varepsilon\right\}$ imply that $\left\|q-q_{a}\right\| \geq \frac{\varepsilon}{2}$. Assume now by contradiction that the infimum over $0<a \leq a_{0}$ is not positive. By continuity of function $\frac{\Psi_{a}(q)-\Psi_{a}\left(q_{a}\right)}{a}$ in $a$ and $q$ when $a>0$ (see Lemma 14), and as the infimum is positive at the limit $a \rightarrow 0$, a non-positive infimum can only be obtained at some $a>0$. It is a contradiction because $q_{a}$ is a well separated minimum for any $a>0$ (Lemma 14).

The infimum in $0 \leq a \leq a_{0}$ is therefore positive for any $q$ such that $q \in \mathbb{S} \cap\left\{\left\|q-q_{0}^{*}\right\| \geq \varepsilon\right\}$. The last set is a compact set in $q$. The infimum over such $q \mathrm{~s}$ is thus positive also.

\section{C.3 Proof of Proposition 12}

By condition $\mathrm{S}$, the relative interiors of sets $B_{U}$ and $\{\gamma=0\}$ have points in common and the infimum is attained at $\lambda_{0}(q)$ (see end of proof, Section B.4). As $\mathbb{S}_{p}$ is compact, denote $\Lambda$ a compact set of $\mathbb{R}^{m}$ such that for all $q \in \mathbb{S}_{p}, \lambda_{0}(q) \in \operatorname{int}(\Lambda)$.

The proof consists in three steps:

1. Under assumption $\mathrm{D}$ that the unconstrained set $B_{U}$ has no faces, the estimate of the unconstrained support function is a consistent and asymptotically Gaussian random process (Proposition 9).

2. The minimization of the estimate $\hat{\delta}_{n}^{*}\left((q, \lambda) \mid B_{U}\right)$ with respect to $\lambda$ holding $q$ constant for any $q$ can be analyzed as in Proposition 10 . 
(a) If $\lambda_{0}(q)$, the minimizer of the true support function, is unique, then any near minimizer in $\lambda$ of $\hat{\delta}_{n}^{*}\left((q, \lambda) \mid B_{U}\right)$ is a $\sqrt{n}$-consistent and asymptotically normal estimate of $\delta^{*}(q \mid B)$.

(b) If $\lambda_{0}(q)$ is not unique, we define a perturbed criterion so as to construct an estimate, $\lambda_{n}(q)$ of one single element $\lambda_{0}^{*}(q)$. Then $\hat{\delta}_{n}^{*}\left(\left(q, \lambda_{n}(q)\right) \mid B_{U}\right)$ is a $\sqrt{n}-$ consistent and asymptotically normal estimate of $\delta^{*}(q \mid B)$.

In both cases, this argument is valid for any finite list of $q$ and the vector of those estimates are jointly asymptotically normal.

3. The derived process $\tau_{n}(q)=\sqrt{n}\left(\hat{\delta}_{n}^{*}\left(\left(q, \lambda_{n}(q)\right) \mid B_{U}\right)-\delta^{*}(q \mid B)\right)$ is stochastically equicontinuous.

Using Andrews (1994, p2251), the three steps prove that $\tau_{n}(q)$ is a consistent and asymptotically Gaussian random process.

Step 1: According to what was developed above, the empirical stochastic process $\tau_{n}^{U}($.$) ,$ defined for $s=(q, \lambda) \in \mathbb{S}_{m}$, the unit sphere in $\mathbb{R}^{m}$, as,

$$
\tau_{n}^{U}(s)=\sqrt{n}\left(\hat{\delta}_{n}^{*}\left(s \mid B_{U}\right)-\delta^{*}\left(s \mid B_{U}\right)\right),
$$

converges to a Gaussian process whose sample paths are uniformly continuous on the unit sphere $\mathbb{S}$, using the usual Euclidean norm. Hence $\tau_{n}^{U}($.$) is stochastically equicontinuous (for instance,$ p.2251 of Andrews, 1994).

Step 2: Fix $q \in \mathbb{S}_{p}$ the unit sphere in $\mathbb{R}^{p}$ and let $\mathcal{S}(q)$ the set of all $s(q)=\left(q, \lambda_{0}(q)\right)$ that minimize $\delta^{*}\left(s \mid B_{U}\right)$ with respect to $\lambda$ i.e.:

$$
\delta^{*}(q \mid B)=\delta^{*}\left(s(q) \mid B_{U}\right)=\min _{\lambda \in \Lambda} \delta^{*}\left(s \mid B_{U}\right) .
$$

$\mathcal{S}(q)$ is a non-empty subset included in the interior of the compact set $S_{B}=\mathbb{S}_{p} \times \Lambda \subset \mathbb{R}_{m}$ by the above. Note also that to obtain the standard evaluation on the unit sphere some renormalization is necessary since $\|s\| \geq\|q\|=1$, and this is done using the positive homogeneity of support functions:

$$
\delta^{*}\left(s \mid B_{U}\right)=\|s\| \delta^{*}\left(\frac{s}{\|s\|} \mid B_{U}\right) .
$$

where $\frac{s}{\|s\|} \in \mathbb{S}_{m}$. In the following, we will directly deal with the support function $\delta^{*}\left(s \mid B_{U}\right)$ extended to the compact set $S_{B}$ in this way.

We first consider the case where $\mathcal{S}(q)$ is a singleton. In the second part of the proof, $\mathcal{S}(q)$ potentially contains more than one element of $\mathbb{R}^{m}$, the issue being to select one specific element of $\mathcal{S}(q)$ and to construct a consistent estimate of it.

a) Suppose that $\mathcal{S}(q)$ is a singleton, $\mathcal{S}(q)=\left\{s_{0}=\left(q, \lambda_{0}\right)\right\} \subset \operatorname{int}\left(S_{B}\right)$.

Let $s_{n}=\left(q, \lambda_{n}\right) \in S_{B}$ be any sequence of directions defined as near minimizers of the empirical counterpart $\hat{\delta}_{n}^{*}\left(s_{n} \mid B_{U}\right)$ defined as,

$$
\hat{\delta}_{n}^{*}\left(s_{n} \mid B_{U}\right) \leq \min _{\lambda \in \Lambda} \hat{\delta}_{n}^{*}\left(s=(q, \lambda) \mid B_{U}\right)+o_{P}(1) .
$$

Define the estimate of $\delta^{*}(q \mid B)$ as the value at the near minimizer:

$$
\hat{\delta}_{n}^{*}(q \mid B)=\hat{\delta}_{n}^{*}\left(s_{n} \mid B_{U}\right) .
$$


First, standard arguments employed for Z-estimators (see van der Vaart, 1998, for instance) imply that:

$$
\operatorname{plim}_{n \rightarrow \infty} \lambda_{n}=\lambda_{0}
$$

Second, because i) $\tau_{n}^{U}($.$) is stochastically equicontinuous ii) s_{n} \in \mathbb{S}_{p} \times \Lambda$ iii) $\operatorname{plim}_{n \rightarrow \infty} s_{n}=s_{0}$, Andrews (1994, equation (3.36), p:2265) shows that:

$$
\sqrt{n}\left(\hat{\delta}_{n}^{*}\left(s_{n} \mid B_{U}\right)-\hat{\delta}_{n}^{*}\left(s_{0} \mid B_{U}\right)\right) \underset{n \rightarrow \infty}{\stackrel{P}{\rightarrow}} 0 .
$$

The step finishes by using the asymptotic distribution of $\hat{\delta}_{n}^{*}\left(s_{0} \mid B_{U}\right)$ :

$$
\sqrt{n}\left(\hat{\delta}_{n}^{*}\left(s_{0} \mid B_{U}\right)-\delta^{*}\left(s_{0} \mid B_{U}\right)\right) \underset{n \rightarrow \infty}{\stackrel{d}{\rightsquigarrow}} N\left(0, V_{s_{0}}\right),
$$

which implies that:

$$
\sqrt{n}\left(\hat{\delta}_{n}^{*}(q \mid B)-\delta^{*}(q \mid B)\right) \underset{n \rightarrow \infty}{\stackrel{d}{\rightsquigarrow}} N\left(0, V_{s_{0}}\right),
$$

using equation (C.24) and where $V_{s_{0}}$ is consistently estimated by $V_{s_{n}}$.

Remark that the same result applies to a finite vector $\left(\hat{\delta}_{n}^{*}\left(q_{1} \mid B\right), \hat{\delta}_{n}^{*}\left(q_{2} \mid B\right), ., \hat{\delta}_{n}^{*}\left(q_{J} \mid B\right)\right)$ using the same arguments.

ii) Suppose now that $\mathcal{S}(q)$ is not a singleton because there are various minimizers of $\delta^{*}(s \mid$ $\left.B_{U}\right)$ in $\lambda$. Note first that set $\mathcal{S}(q) \subset \operatorname{int}\left(S_{B}\right)$ is convex and compact because $\delta^{*}\left(s \mid B_{U}\right)$ is convex and continuous. We are first going to select and characterize a unique $\left(q, \lambda_{0}^{*}\right)$ from $\mathcal{S}(q)$. Consider the smallest convex cone which includes $\mathcal{S}(q)$ :

$$
\mathcal{C S}(q)=\left\{c . s_{0} ; s_{0} \in \mathcal{S}(q), c \geq 0\right\} .
$$

and consider the projection of $(q, 0)$ on $\mathcal{C S}(q)$. This projection is unique and defined by $c^{*} s_{0}^{*}$ where $\left(c^{*}, s_{0}^{*}\right)$ is the argument of the minimum:

$$
\min _{(c \geq 0,(q, \lambda) \in \mathcal{S}(q))}\|((1-c) q,-c \lambda)\|^{2}=\min _{(c \geq 0, s \in \mathcal{S}(q))}\left\{(1-c)^{2}+c^{2} \lambda^{\top} \lambda\right\}
$$

since $q^{\top} q=1$. It yields $c^{*}=\frac{1}{1+\lambda^{\top} \lambda}>0$ whereas $\lambda_{0}^{*}$ is the argument of:

$$
\min _{\lambda,(q, \lambda) \in \mathcal{S}(q)} \lambda^{\top} \lambda
$$

Vector $\lambda_{0}^{*}$ is unique because it is a (normalized) projection. Given this fact, we can define a sequence of perturbed programs such that $s_{0}^{*}$ corresponds to the limit of the sequence of minima. Specifically, for any $a>0$, let :

$$
\Psi_{a}(s)=\delta^{*}\left(s \mid B_{U}\right)+a \lambda^{\top} \lambda
$$

Because $\delta^{*}\left(s \mid B_{U}\right)$ is convex in $\lambda$ and $\lambda^{\top} \lambda$ is strictly convex in $\lambda, \Psi_{a}(s)$ is a strictly convex function in $\lambda$.

The minimum $s_{a}=\left(q, \lambda_{a}\right)$ of $\Psi_{a}(s)$ is unique because we minimize a strictly convex function on a compact set $S_{B}$. Furthermore, we now show that $\lambda_{a}$ tends to $\lambda_{0}^{*}$ when $a \rightarrow 0$. 
Lemma 17 The limit of the sequence $\left\{\lambda_{a}\right\}_{a>0}$ exists when $a \rightarrow 0$ and is equal to $\lambda_{0}^{*}$.

Proof. To begin with, it is useful to note that $\delta^{*}\left(s_{0}^{*} \mid B_{U}\right)$ provides a lower bound for $\Psi_{a}(s)$,

$$
\Psi_{a}(s) \geq \delta^{*}\left(s_{0}^{*} \mid B_{U}\right),
$$

because $s_{0}^{*}$ is a minimizer of $\delta^{*}\left(s \mid B_{U}\right)$.

We consider two cases:

- Assume first that $(q, 0) \in \mathcal{S}(q)$. In such a case, $\lambda_{0}^{*}=0$ and $\Psi_{a}\left(s_{0}^{*}\right)=\delta^{*}\left(s_{0}^{*} \mid B_{U}\right)$. Hence, given that $\lambda_{a}$ is unique and that $\delta^{*}\left(s_{0}^{*} \mid B_{U}\right)$ is a lower bound for $\Psi_{a}(s)$, we have necessarily $s_{a}=s_{0}^{*}$ for any $a>0$ and hence $\lambda_{a}=\lambda_{0}^{*}$.

- Assume now that $(q, 0) \notin \mathcal{S}(q)$. By definition of $\lambda_{a}$ as a minimum,

$$
\begin{aligned}
\Psi_{a}\left(s_{a}\right) & =\delta^{*}\left(s_{a} \mid B_{U}\right)+a \lambda_{a}^{\top} \lambda_{a} \\
& \leq \Psi_{a}\left(s_{0}^{*}\right)=\delta^{*}\left(s_{0}^{*} \mid B_{U}\right)+a \lambda_{0}^{* \top} \lambda_{0},
\end{aligned}
$$

It implies that:

$$
0 \leq \delta^{*}\left(s_{a} \mid B_{U}\right)-\delta^{*}\left(s_{0}^{*} \mid B_{U}\right) \leq a\left(\lambda_{0}^{* \top} \lambda_{0}^{*}-\lambda_{a}^{\top} \lambda_{a}\right) \leq a \lambda_{0}^{* \top} \lambda_{0}^{*},
$$

so that the distance between $s_{a}$ and the set $\mathcal{S}(q)=\left\{s ; \delta^{*}\left(s \mid B_{U}\right)=\delta^{*}\left(s_{0}^{*} \mid B_{U}\right)\right\}$ tends to zero when $a$ tends to zero by the continuity of function $\delta^{*}\left(s \mid B_{U}\right)$.

Consider now $\lambda_{m}$ any accumulation point of the sequence $\lambda_{a}$ i.e., any point satisfying, $\forall \eta>0, \exists a>0$ such that $\left\|\lambda_{a}-\lambda_{m}\right\|<\eta$. Because $\mathcal{S}(q)$ is compact, $s_{m}=\left(q, \lambda_{m}\right) \in$ $\mathcal{S}(q)$. We are going to show that $s_{m}=s_{0}^{*}$. By definition of $\lambda_{a}$ and $\lambda_{0}^{*}$, we have

$$
\frac{\Psi_{a}\left(s_{a}\right)-\delta^{*}\left(s_{0}^{*} \mid B_{U}\right)}{a} \leq \frac{\Psi_{a}\left(s_{0}^{*}\right)-\delta^{*}\left(s_{0}^{*} \mid B_{U}\right)}{a}=\lambda_{0}^{* \top} \lambda_{0}^{*} \leq \lambda_{m}^{\top} \lambda_{m} .
$$

where the first inequality holds true because $s_{a}$ minimizes $\Psi_{a}$ whereas the second inequality holds true because $s_{m} \in \mathcal{S}(q)$ is compact and $\lambda_{0}^{*}$ minimizes $\lambda_{0}^{* \top} \lambda_{0}^{*}$ on $\mathcal{S}(q)$. Furthermore, because $s_{0}^{*} \in \mathcal{S}(q)$

$$
\lambda_{a}^{\top} \lambda_{a}=\frac{\Psi_{a}\left(s_{a}\right)-\delta^{*}\left(s_{a} \mid B_{U}\right)}{a} \leq \frac{\Psi_{a}\left(s_{a}\right)-\delta^{*}\left(s_{0}^{*} \mid B_{U}\right)}{a} .
$$

Combining the two equations,

$$
\lambda_{a}^{\top} \lambda_{a} \leq \lambda_{0}^{* \top} \lambda_{0}^{*} \leq \lambda_{m}^{\top} \lambda_{m}
$$

By taking limits and using that $\lambda_{a}$ tends to $\lambda_{m}$ when $a$ tends to zero, we obtain that $\lambda_{m}=$ $\lambda_{0}^{*}$. We thus have shown that:

$$
\lim _{a \rightarrow 0}\left\|\lambda_{a}-\lambda_{0}^{*}\right\|=0
$$

Furthermore, we check:

$$
0 \leq \frac{\Psi_{a}\left(s_{0}^{*}\right)-\Psi_{a}\left(s_{a}\right)}{a} \leq \lambda_{0}^{* \top} \lambda_{0}^{*}-\lambda_{a}^{\top} \lambda_{a}
$$

so that, since $\lambda_{a} \rightarrow \lambda_{0}^{*}$ when $a \rightarrow 0$ :

$$
\Psi_{a}\left(s_{0}^{*}\right)-\Psi_{a}\left(s_{a}\right)=o(a) .
$$


The next step is to construct an estimate of $\lambda_{a}$. Before moving on to this step, we are going to prove a lemma that will be useful for showing that the estimate of $\lambda_{a}$ actually converges to $\lambda_{0}^{*}$.

\section{Lemma 18}

$$
\forall \varepsilon>0, \exists a_{0}>0, \exists \eta>0, \forall a \text { such that } \inf _{0<a \leq a_{0},\left\|\lambda-\lambda_{0}^{*}\right\| \geq \varepsilon} \frac{\Psi_{a}(s)-\Psi_{a}\left(s_{a}\right)}{a}>\eta .
$$

Proof. First, the Lemma is trivially satisfied when $s_{0}^{*}=(q, 0)$ since $\lambda_{a}=\lambda_{0}^{*}=0$ for any $a$ and therefore:

$$
\frac{\Psi_{a}(s)-\Psi_{a}\left(s_{a}\right)}{a}=\lambda^{T} \lambda
$$

which is bounded from below by $\varepsilon^{2}$ when $\|\lambda\| \geq \varepsilon$.

Assume that $(q, 0) \notin \mathcal{S}(q)$. Like for Lemma 16, we will first show that the infimum is positive for a given $q$ and then use the compactness of the space where $q$ evolves to conclude. We know from the previous Lemma that $\lambda \rightarrow \lambda_{0}^{*}$ when $a$ tends to 0 .

- When $s=(q, \lambda) \in \mathcal{S}(q), \frac{\Psi_{a}(s)-\Psi_{a}\left(s_{a}\right)}{a} \rightarrow \lambda^{T} \lambda-\lambda_{0}^{* \top} \lambda_{0}$ when $a$ tends to zero; which is strictly positive when $\left\|\lambda-\lambda_{0}^{*}\right\| \geq \varepsilon$.

- When $s=(q, \lambda) \notin \mathcal{S}(q), \frac{\Psi_{a}(s)-\Psi_{a}\left(s_{a}\right)}{a} \rightarrow+\infty$ when $a$ tends to zero and cannot deliver the infimum.

As $\lambda_{a} \rightarrow \lambda_{0}^{*}$ when $a$ tends to zero, there exists some positive $a_{0}$ such that the joint events $\left\{0<a<a_{0}\right\}$ and $\left\{\left\|\lambda-\lambda_{0}^{*}\right\| \geq \varepsilon\right\}$ implies that $\left\|\lambda-\lambda_{a}\right\| \geq \varepsilon / 2$. Assume now by contradiction that the infimum over $0<a<a_{0}$ is not positive. By continuity of function $\frac{\Psi_{a}(s)-\Psi_{a}\left(s_{a}\right)}{a}$ in $a$ and $s$ when $a>0$, and as the infimum is positive at the limit $a \rightarrow 0$, a non-positive infimum can only be obtained at some $a>0$ and $s_{a} \in S_{B}$. It is a contradiction because $s_{a}$ is a well separated minimum for any $a>0$. The compactness of $S_{B} \cap\left\{s \in S_{B},\left\|\lambda-\lambda_{0}^{*}\right\| \geq \varepsilon\right\}$ ensures that the infimum over such $s \mathrm{~s}$ in this set is positive also.

Finally, we construct the estimate of $\lambda_{a}$. Fix $a>0$. Define the perturbed estimated program as:

$$
\Psi_{n, a}(s)=\hat{\delta}_{n}^{*}\left(s \mid B_{U}\right)+a \lambda^{\top} \lambda .
$$

and restrict the set over which we take the supremum as $s \in S_{B}$

Define $s_{n, a}$ as a near minimizer of $\Psi_{n, a}$ over $S_{B}$. We can adapt the same kind of argument than the one used in the proof of Proposition 10 to show that

$$
\frac{\Psi_{a}\left(s_{n, a}\right)-\Psi_{a}\left(s_{a}\right)}{a} \leq \frac{\sup _{s \in S_{B}}\left|\delta^{*}\left(s \mid B_{U}\right)-\hat{\delta}_{n}^{*}\left(s \mid B_{U}\right)\right|+O_{P}\left(n^{-1 / 2}\right)}{a} .
$$

Let $a_{n}=O_{P}\left(n^{-\alpha}\right)$ where $\alpha<1 / 2$. Because of equicontinuity and $n^{1 / 2}$ convergence of $\hat{\delta}_{n}^{*}\left(s \mid B_{U}\right)$ to $\delta^{*}\left(s \mid B_{U}\right)$, when $s \in S_{B}$, we have that:

$$
n^{\alpha} \sup _{s \in S_{B}}\left|\delta^{*}\left(s \mid B_{U}\right)-\hat{\delta}_{n}^{*}\left(s \mid B_{U}\right)\right| \underset{n \rightarrow \infty}{\stackrel{P}{\rightarrow}} 0 .
$$


Then:

$$
\frac{\Psi_{a_{n}}\left(s_{n, a_{n}}\right)-\Psi_{a_{n}}\left(s_{a_{n}}\right)}{a_{n}} \leq o_{P}(1)
$$

We thus have:

$$
\forall \eta, \lim _{n \rightarrow \infty} \operatorname{Pr}\left(\frac{\Psi_{a_{n}}\left(s_{n, a_{n}}\right)-\Psi_{a_{n}}\left(s_{a_{n}}\right)}{a_{n}}>\eta\right)=0 .
$$

By condition (C.27), for any $\varepsilon$, there exist $\eta>0$ and $n_{0}$ such that for any $n \geq n_{0}$ the event:

$$
\left\{d\left(s_{n, a_{n}}, s_{0}^{*}\right) \geq \varepsilon\right\} \subset\left\{\frac{\Psi_{a_{n}}\left(s_{n, a_{n}}\right)-\Psi_{a_{n}}\left(s_{a_{n}}\right)}{a_{n}}>\eta\right\} .
$$

Therefore:

$$
\forall \varepsilon, \lim _{n \rightarrow \infty} \operatorname{Pr}\left(d\left(s_{n, a_{n}}, s_{0}^{*}\right)>\varepsilon\right)=0 \Longrightarrow s_{n, a_{n}}-s_{0}^{*} \underset{n \rightarrow \infty}{\stackrel{P}{\rightarrow}} 0 .
$$

Then the same argument than in part ( $i)$ applies and:

$$
\sqrt{n}\left(\hat{\delta}_{n}^{*}\left(s_{a_{n}, n} \mid B_{U}\right)-\hat{\delta}_{n}^{*}\left(s_{0}^{*} \mid B_{U}\right)\right) \underset{n \rightarrow \infty}{\stackrel{P}{\rightarrow}} 0 .
$$

We can then use the asymptotic distribution of $\hat{\delta}_{n}^{*}\left(s_{0}^{*} \mid B_{U}\right)$ in place of $\hat{\delta}_{n}^{*}\left(s_{a_{n}, n} \mid B_{U}\right)$.

By the same development, it applies to a finite vector of such estimates defined at values $q_{1}, q_{2}, . ., q_{J}$

Step 3: We now turn to equicontinuity. As the process $\tau_{n}^{U}(s)$ is equicontinuous, we know that for any $\varepsilon>0$ and $\eta>0$ there exists $\delta$ such that:

$$
\lim _{n \rightarrow \infty} \operatorname{Pr}\left[\sup _{s_{1}, s_{2} \in \mathbb{S}_{m},\left\|s_{1}-s_{2}\right\|<\delta}\left|\tau_{n}^{U}\left(s_{1}\right)-\tau_{n}^{U}\left(s_{2}\right)\right|>\eta\right]<\varepsilon .
$$

It is straightforward to extend this result to the compact set $S_{B}=\mathbb{S}_{p} \times \Lambda$ so that:

$$
\forall \varepsilon, \forall \eta, \exists \delta>0, \lim _{n \rightarrow \infty} \operatorname{Pr}\left[\sup _{s_{1}, s_{2} \in S_{B},\left\|s_{1}-s_{2}\right\|<\delta}\left|\tau_{n}^{U}\left(s_{1}\right)-\tau_{n}^{U}\left(s_{2}\right)\right|>\eta\right]<\varepsilon .
$$

Let $s_{1 n}$ and $s_{2 n}$ be defined as:

$$
\hat{\delta}_{n}^{*}\left(s_{1 n} \mid B_{U}\right)=\hat{\delta}_{n}^{*}\left(q_{1}, \lambda_{n}\left(q_{1}\right) \mid B_{U}\right), \hat{\delta}_{n}^{*}\left(s_{2 n} \mid B_{U}\right)=\hat{\delta}_{n}^{*}\left(q_{2}, \lambda_{n}\left(q_{2}\right) \mid B_{U}\right)
$$

where for $j=1,2, \lambda_{n}\left(q_{j}\right)$ are minimizers of $\hat{\delta}_{n}^{*}\left(q_{j}, \lambda_{n}\left(q_{j}\right) \mid B_{U}\right)$ defined as:

$$
\hat{\delta}_{n}^{*}\left(s_{j n} \mid B_{U}\right)=\min _{\lambda \in \Lambda} \hat{\delta}_{n}^{*}\left(s_{j} \mid B_{U}\right)=\min _{\lambda \in \Lambda} \hat{\delta}_{n}^{*}\left(\left(q_{j}, \lambda\right) \mid B_{U}\right),
$$

if they are unique or by the argument used in Step 2 b) if they are not. Consider the difference:

$$
\begin{aligned}
\hat{\delta}_{n}^{*}\left(s_{1 n} \mid\right. & \left.B_{U}\right)-\hat{\delta}_{n}^{*}\left(s_{2 n} \mid B_{U}\right)=\min _{\lambda \in \Lambda} \hat{\delta}_{n}^{*}\left(\left(q_{1}, \lambda\right) \mid B_{U}\right)-\min _{\lambda \in \Lambda} \hat{\delta}_{n}^{*}\left(\left(q_{2}, \lambda\right) \mid B_{U}\right) \\
& =\min _{\lambda \in \Lambda}\left(\hat{\delta}_{n}^{*}\left(\left(q_{1}, \lambda\right) \mid B_{U}\right)-\hat{\delta}_{n}^{*}\left(\left(q_{2}, \lambda\right) \mid B_{U}\right)+\hat{\delta}_{n}^{*}\left(\left(q_{2}, \lambda\right) \mid B_{U}\right)\right)-\min _{\lambda \in \Lambda} \hat{\delta}_{n}^{*}\left(\left(q_{2}, \lambda\right) \mid B_{U}\right) \\
& \geq \min _{\lambda \in \Lambda}\left(\hat{\delta}_{n}^{*}\left(\left(q_{1}, \lambda\right) \mid B_{U}\right)-\hat{\delta}_{n}^{*}\left(\left(q_{2}, \lambda\right) \mid B_{U}\right)\right) .
\end{aligned}
$$


Or alternatively:

$$
\begin{aligned}
\hat{\delta}_{n}^{*}\left(s_{1 n} \mid\right. & \left.B_{U}\right)-\hat{\delta}_{n}^{*}\left(s_{2 n} \mid B_{U}\right)=\min _{\lambda \in \Lambda} \hat{\delta}_{n}^{*}\left(\left(q_{1}, \lambda\right) \mid B_{U}\right)-\min _{\lambda} \hat{\delta}_{n}^{*}\left(\left(q_{2}, \lambda\right) \mid B_{U}\right) \\
& =\min _{\lambda \in \Lambda} \hat{\delta}_{n}^{*}\left(\left(q_{1}, \lambda\right) \mid B_{U}\right)-\min _{\lambda \in \Lambda}\left(\hat{\delta}_{n}^{*}\left(\left(q_{1}, \lambda\right) \mid B_{U}\right)-\hat{\delta}_{n}^{*}\left(\left(q_{1}, \lambda\right) \mid B_{U}\right)+\hat{\delta}_{n}^{*}\left(\left(q_{2}, \lambda\right) \mid B_{U}\right)\right) \\
& \leq-\min _{\lambda \in \Lambda}\left(-\hat{\delta}_{n}^{*}\left(\left(q_{1}, \lambda\right) \mid B_{U}\right)+\hat{\delta}_{n}^{*}\left(\left(q_{2}, \lambda\right) \mid B_{U}\right)\right) \\
& =\max _{\lambda \in \Lambda}\left(\hat{\delta}_{n}^{*}\left(\left(q_{1}, \lambda\right) \mid B_{U}\right)-\hat{\delta}_{n}^{*}\left(\left(q_{2}, \lambda\right) \mid B_{U}\right)\right) .
\end{aligned}
$$

In consequence:

$$
\left|\hat{\delta}_{n}^{*}\left(s_{1 n} \mid B_{U}\right)-\hat{\delta}_{n}^{*}\left(s_{2 n} \mid B_{U}\right)\right| \leq \max _{\lambda \in \Lambda}\left|\hat{\delta}_{n}^{*}\left(\left(q_{1}, \lambda\right) \mid B_{U}\right)-\hat{\delta}_{n}^{*}\left(\left(q_{2}, \lambda\right) \mid B_{U}\right)\right| .
$$

By definition:

$$
\tau_{n}\left(q_{1}\right)-\tau_{n}\left(q_{2}\right)=\tau_{n}^{U}\left(s_{1 n}\right)-\tau_{n}^{U}\left(s_{2 n}\right)=\sqrt{n}\left(\hat{\delta}_{n}^{*}\left(s_{1 n} \mid B_{U}\right)-\hat{\delta}_{n}^{*}\left(s_{2 n} \mid B_{U}\right)\right)
$$

so that for any $(\varepsilon, \eta)$ and for any $\delta$ satisfying equation (C.28) :

$$
\begin{aligned}
& \lim _{n \rightarrow \infty} \operatorname{Pr}\left[\sup _{\left\|q_{1}-q_{2}\right\|<\delta}\left|\tau_{n}\left(q_{1}\right)-\tau_{n}\left(q_{2}\right)\right|>\eta\right] \\
= & \lim _{n \rightarrow \infty} \operatorname{Pr}\left[\sup _{\left\|q_{1}-q_{2}\right\|<\delta}\left|\sqrt{n}\left(\hat{\delta}_{n}^{*}\left(s_{1 n} \mid B_{U}\right)-\hat{\delta}_{n}^{*}\left(s_{2 n} \mid B_{U}\right)\right)\right|>\eta\right] \\
\leq & \lim _{n \rightarrow \infty} \operatorname{Pr}\left[\sup _{\left\|q_{1}-q_{2}\right\|<\delta} \sqrt{n} \max _{\lambda \in \Lambda}\left|\hat{\delta}_{n}^{*}\left(\left(q_{1}, \lambda\right) \mid B_{U}\right)-\hat{\delta}_{n}^{*}\left(\left(q_{2}, \lambda\right) \mid B_{U}\right)\right|>\eta\right] \\
= & \lim _{n \rightarrow \infty} \operatorname{Pr}\left[\sup _{s_{1}, s_{2} \in S_{B},\left\|s_{1}-s_{2}\right\|<\delta}\left|\sqrt{n}\left(\hat{\delta}_{n}^{*}\left(\left(q_{1}, \lambda\right) \mid B_{U}\right)-\hat{\delta}_{n}^{*}\left(\left(q_{2}, \lambda\right) \mid B_{U}\right)\right)\right|>\eta\right] \\
= & \lim _{n \rightarrow \infty} \operatorname{Pr}\left[\sup _{s_{1}, s_{2} \in S_{B},\left\|s_{1}-s_{2}\right\|<\delta}\left|\sqrt{n}\left(\hat{\delta}_{n}^{*}\left(s_{1} \mid B_{U}\right)-\hat{\delta}_{n}^{*}\left(s_{2} \mid B_{U}\right)\right)\right|>\eta\right]<\varepsilon .
\end{aligned}
$$

that proves that the process $\tau_{n}(q)$ is equicontinuous by equation (C.28).

The proof when the minimizers are replaced by near-minimizers can be adapted in a straightforward way.

\section{Computations of Section 5}

\section{D.1 Example of Section 5.1}

The simulated model is:

$$
y^{*}=0 . x_{1}+0 . x_{2}+\varepsilon
$$

We compute $\delta^{*}(q \mid B)$ using $z=x$ as instruments. As $\Sigma^{-1}=E\left(x^{\top} x\right)=I_{2}$, we have:

$$
\left\{\begin{array}{c}
z_{q}=x q=\cos \theta x_{1}+\sin \theta x_{2} \\
w_{q}=y-\Delta+2 \Delta \mathbf{1}\left\{z_{q}>0\right\}
\end{array}\right.
$$


Using

we obtain:

$$
\left(\begin{array}{l}
x_{1} \\
x_{2} \\
z_{q}
\end{array}\right) \sim \mathcal{N}\left(\left[\begin{array}{l}
0 \\
0 \\
0
\end{array}\right],\left[\begin{array}{ccc}
1 & 0 & \cos \theta \\
0 & 1 & \sin \theta \\
\cos \theta & \sin \theta & 1
\end{array}\right]\right)
$$

and therefore:

$$
E x_{1} \mathbf{1}_{z_{q}>0}=\frac{1}{\sqrt{2 \pi}} \cos \theta \text { and } E x_{2} \mathbf{1}_{z_{q}>0}=\frac{1}{\sqrt{2 \pi}} \sin \theta
$$

$$
\delta^{*}(q \mid B)=E\left(z_{q} w_{q}\right)=\frac{2 \Delta}{\sqrt{2 \pi}} .
$$

The frontier points are:

$$
\beta_{q}=E\left(x^{\top} w_{q}\right)=\frac{2 \Delta}{\sqrt{2 \pi}}\left[\begin{array}{c}
\cos \theta \\
\sin \theta
\end{array}\right]
$$

\section{D.2 Example of Section 5.2}

The simulated model is:

$$
y^{*}=0 . x_{1}+0 . x_{2}+\varepsilon
$$

$x_{2}=\pi e_{2}+\sqrt{1-\pi^{2}} e_{3}, w=\nu e_{3}+\sqrt{1-\nu^{2}} e_{4}$ where $\left(e_{2}, e_{3}, e_{4}\right)$ is a standard unit normal vector. It is convenient to define $\mu=\nu \sqrt{1-\pi^{2}}$ and $a^{2}=\pi^{2}+\mu^{2}=\pi^{2}+\nu^{2}\left(1-\pi^{2}\right)$.

To conform with general notations, let $x=\left(x_{1}, x_{2}\right)$ and $z=\left(x_{1}, e_{2}, w\right)$. As there exists one supernumerary restriction, we first evaluate $z_{F}$ and $z_{H}$ as defined in Appendix B. As $E\left(z^{\top} z\right)=$ $I_{3}$, we have:

$$
E\left(x^{\top} z\right)=\left(\begin{array}{ccc}
1 & 0 & 0 \\
0 & \pi & \mu
\end{array}\right), \quad\left[E\left(x^{\top} z\right) E\left(z^{\top} z\right)^{-1} E\left(z^{\top} x\right)\right]^{-1 / 2}=\left(\begin{array}{cc}
1 & 0 \\
0 & a^{-1}
\end{array}\right)
$$

and:

$$
z_{F}^{\top}=\left[E\left(x^{\top} z\right)\left(E\left(z^{\top} z\right)\right)^{-1} E\left(z^{\top} x\right)\right]^{-1 / 2} E\left(x^{\top} z\right) E\left(z^{\top} z\right)^{-1} z^{\top}=\left(\begin{array}{c}
x_{1} \\
\frac{\pi e_{2}+\mu w}{a}
\end{array}\right),
$$

which is standard unit bivariate normally distributed. Moreover as:

$$
E\left(z_{F}^{\top} z\right)=\left(\begin{array}{ccc}
1 & 0 & 0 \\
0 & \frac{\pi}{a} & \frac{\mu}{a}
\end{array}\right)
$$

the normalized vector $\left(\begin{array}{ccc}0 & \frac{\mu}{a}-\frac{\pi}{a}\end{array}\right)^{\top}$ belongs to the kernel of this operator and in consequence, $z_{H}=\frac{\mu e_{2}-\pi w}{a}$.

To construct $B_{U}$, we use $\left(z_{F}, z_{H}\right)$ and we write:

$$
\Sigma^{\top}=\left[E\left(\begin{array}{c}
x_{1} \\
a^{-1}\left(\pi e_{2}+\mu w\right) \\
z_{H}
\end{array}\right)\left(\begin{array}{lll}
x_{1} & x_{2} & z_{H}
\end{array}\right)\right]^{-1}=\left(\begin{array}{ccc}
1 & 0 & 0 \\
0 & a^{-1} & 0 \\
0 & 0 & 1
\end{array}\right) .
$$

Let $q=\left(q_{1}, q_{2}\right)^{\top}$ such that $q_{1}^{2}+q_{2}^{2}=1$ and define:

$$
\begin{aligned}
z_{q, \lambda} & =\left(q^{\top} \lambda\right)\left(\begin{array}{c}
x_{1} \\
a^{-2}\left(\pi e_{2}+\mu w\right) \\
z_{H}
\end{array}\right) \\
& =x_{1} q_{1}+\left(a^{-2}\left(\pi e_{2}+\mu w\right)\right) q_{2}+z_{H} \lambda .
\end{aligned}
$$


The variance of $z_{q, \lambda}$ is therefore

$$
V_{q, \lambda}=q_{1}^{2}+\frac{q_{2}^{2}}{a^{2}}+\lambda^{2}
$$

As in the previous example,

$$
w_{q, \lambda}=y-\Delta+2 \Delta \mathbf{1}\left\{z_{q, \lambda}>0\right\} .
$$

The covariances of $z_{q, \lambda}$ with the variables of interest are:

$$
E\left(z_{q, \lambda} x_{1}\right)=q_{1}, E\left(z_{q, \lambda}\left(a^{-1}\left(\pi e_{2}+\mu w\right)\right)\right)=a^{-1} q_{2}, E\left(z_{q, \lambda} z_{H}\right)=\lambda,
$$

so that for instance,

$$
E x_{1} \mathbf{1}_{z_{q, \lambda}>0}=\frac{1}{\sqrt{2 \pi}} \frac{q_{1}}{\sqrt{V_{q, \lambda}}}
$$

using the normality assumptions. In consequence, a closed-form expression for $\delta^{*}\left(q, \lambda \mid B_{U}\right)$ is:

$$
\delta^{*}\left(q, \lambda \mid B_{U}\right)=\frac{2 \Delta}{\sqrt{2 \pi}} \sqrt{q_{1}^{2}+\frac{q_{2}^{2}}{a^{2}}+\lambda^{2}} .
$$

This function is minimized when $\lambda=0$ and $B_{U}$ is an ellipsoid orthogonal to the hyperplane $\gamma=0$. Its projection on the hyperplane is also an ellipse and the identified set is an ellipse:

$$
\delta^{*}(q \mid B)=\frac{2 \Delta}{\sqrt{2 \pi}} \sqrt{q_{1}^{2}+\frac{q_{2}^{2}}{a^{2}}} .
$$

\section{D.3 Example of section 5.3}

The simulated model is:

$$
y^{*}=\frac{1}{2}+\frac{x}{8}+\varepsilon
$$

and variable $z \equiv\left(1, x_{1}\right)^{\top}$ are the instruments. As $\Sigma=\left(E\left(z^{\top} z\right)\right)^{-1}=I_{2}$, we can derive the variables of interest:

$$
\left\{\begin{array}{l}
z_{q}=z \Sigma q=\cos \theta+x \sin \theta \\
w_{q}=y+\frac{1}{2} \mathbf{1}\left\{z_{q}>0\right\} \\
\underline{y}=\frac{1}{2}\left\{y^{*} \geq 0.5\right\}
\end{array}\right.
$$

$E(\underline{y})=\frac{1}{4}$ and $E(x \underline{y})=\frac{1}{8}$ so we can derive the frontier points $\beta_{q}$ :

$$
\begin{aligned}
\beta_{q} & =\Sigma E\left(z^{\top} w_{q}\right)=E\left(z^{\top} \underline{y}\right)+\frac{1}{2} E\left(z^{\top} \mathbf{1}\left\{z_{q}>0\right\}\right. \\
& =\left[\begin{array}{c}
\frac{1}{4} \\
\frac{1}{8}
\end{array}\right]+\left[\begin{array}{c}
\frac{1}{2} E\left(\mathbf{1}\left\{z_{q}>0\right\}\right) \\
\frac{1}{2} E\left(x_{1} \mathbf{1}\left\{z_{q}>0\right\}\right)
\end{array}\right] .
\end{aligned}
$$

Let $\theta_{0}=\pi / 4$. For $\theta$ being between $-\theta_{0}$ and $\theta_{0} z_{q}$ is always positive whatever the value of $x$ :

$$
\begin{aligned}
E 1\left\{z_{q}>0\right\} & =1 \\
\operatorname{Ex} 1\left\{z_{q}>0\right\} & =0
\end{aligned}
$$

and $\beta_{q}=\left[\frac{3}{4} ; \frac{1}{8}\right]^{\top}$. 
For $\theta$ being between $\theta_{0}$ and $-\theta_{0}+\pi, z_{q}$ is negative when $x=-1$, otherwise positive:

$$
\begin{aligned}
\operatorname{E1}\left\{z_{q}>0\right\} & =\frac{1}{2} \\
\operatorname{Ex} \mathbf{1}\left\{z_{q}>0\right\} & =\frac{1}{2}
\end{aligned}
$$

and $\beta_{q}=\left[\frac{1}{2} ; \frac{3}{8}\right]^{\top}$.

We obtain similarly $\beta_{q}=\left[\frac{1}{4} ; \frac{1}{8}\right]^{\top}$ when $\theta$ is between $\theta_{0}+\pi$ and $\theta_{0}+\pi$ and $\beta_{q}=\left[\frac{1}{2} ;-\frac{1}{8}\right]^{\top}$ for $\theta$ being between $\theta_{0}-\pi$ and $-\theta_{0}$.

The term $\tau_{1}(q)$ defined in proposition 9 is equal to zero when $P\left(z_{q}=0\right)=0$, i.e. when $\theta \neq \frac{(2 k+1) \Pi}{4}$. When $\theta=\Pi / 4, z_{q}=0$ when $x=-1$ which occurs with probability $1 / 2$. However the term $q^{\top}\left(\hat{\Sigma}_{n}-\Sigma\right) z^{\top}$ is equal to $\frac{1}{\sqrt{2}}(1+x) \frac{1}{n} \sum_{i=1}^{n} x_{i}$ which is equal to zero when $x=-1$. $\tau_{1}(q)$ the additional term in the asymptotic distribution is therefore equal to zero. The proof is similar for other values of $\theta$. 


\section{Figures and Tables}

Table 1: Results related to the Monte Carlo simulations - example 1

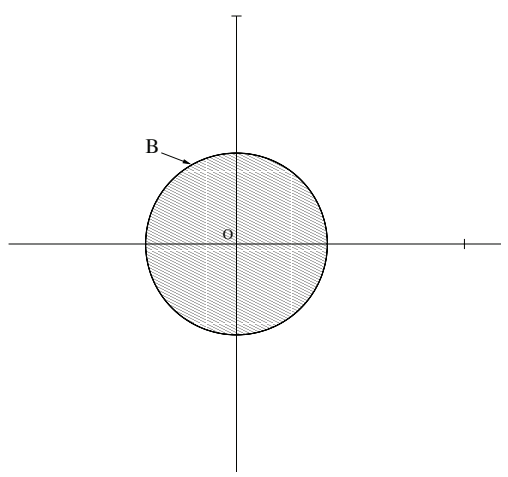

Set B, $y=0 . x_{1}+0 . x_{2}+\varepsilon,\left(x_{1}, x_{2}\right)^{T} \sim N\left(0, I_{2}\right)$

Support function $\delta(q)$ for $q=(0,1)^{T}$

True unknown value 0.199

\begin{tabular}{ccccc}
\hline \hline $\mathrm{n}$ & Mean & $\mathrm{Q} 1$ & $\mathrm{Q} 2$ & $\mathrm{Q} 3$ \\
\hline 100 & 0.198 & 0.178 & 0.197 & 0.216 \\
500 & 0.199 & 0.190 & 0.199 & 0.208 \\
1000 & 0.199 & 0.193 & 0.199 & 0.206 \\
2500 & 0.199 & 0.196 & 0.199 & 0.203 \\
\hline \hline
\end{tabular}


Table 2: Percentage of rejections for the two tests - first example

\begin{tabular}{|c|c|c|c|c|c|c|c|c|}
\hline \multirow[b]{2}{*}{$\mathrm{r}$} & \multicolumn{4}{|c|}{$\begin{array}{c}\text { Test } 1 \\
\left(H_{0}: \beta^{r} \in B\right)\end{array}$} & \multicolumn{4}{|c|}{$\begin{array}{c}\text { Test } 2 \\
\left(H_{0}: \beta^{r} \in \partial B\right)\end{array}$} \\
\hline & $n=100$ & $n=500$ & $n=1000$ & $n=2500$ & $n=100$ & $n=500$ & $n=1000$ & $n=2500$ \\
\hline 0.01 & $0 \%$ & $0 \%$ & $0 \%$ & $0 \%$ & $70.9 \%$ & $100 \%$ & $100 \%$ & $100 \%$ \\
\hline 0.05 & $0 \%$ & $0 \%$ & $0 \%$ & $0 \%$ & $69.9 \%$ & $100 \%$ & $100 \%$ & $100 \%$ \\
\hline 0.1 & $0 \%$ & $0 \%$ & $0 \%$ & $0 \%$ & $67.7 \%$ & $100 \%$ & $100 \%$ & $100 \%$ \\
\hline 0.2 & $0 \%$ & $0 \%$ & $0 \%$ & $0 \%$ & $60.1 \%$ & $100 \%$ & $100 \%$ & $100 \%$ \\
\hline 0.3 & $0 \%$ & $0 \%$ & $0 \%$ & $0 \%$ & $51.6 \%$ & $99.9 \%$ & $100 \%$ & $100 \%$ \\
\hline 0.4 & $0 \%$ & $0 \%$ & $0 \%$ & $0 \%$ & $40.5 \%$ & $99.6 \%$ & $100 \%$ & $100 \%$ \\
\hline 0.5 & $0 \%$ & $0 \%$ & $0 \%$ & $0 \%$ & $29.4 \%$ & $97.3 \%$ & $99.9 \%$ & $100 \%$ \\
\hline 0.6 & $0.5 \%$ & $0 \%$ & $0 \%$ & $0 \%$ & $19.6 \%$ & $85.4 \%$ & $99 \%$ & $100 \%$ \\
\hline 0.65 & $0.7 \%$ & $0 \%$ & $0 \%$ & $0 \%$ & $16.2 \%$ & $73.3 \%$ & $97.1 \%$ & $100 \%$ \\
\hline 0.7 & $1 \%$ & $0 \%$ & $0 \%$ & $0 \%$ & $12.7 \%$ & $61.1 \%$ & $89.8 \%$ & $99.9 \%$ \\
\hline 0.75 & $1.3 \%$ & $0.1 \%$ & $0 \%$ & $0 \%$ & $9.7 \%$ & $45.8 \%$ & $76.2 \%$ & $99 \%$ \\
\hline 0.8 & $1.6 \%$ & $0.1 \%$ & $0 \%$ & $0 \%$ & $7.9 \%$ & $31.5 \%$ & $58.2 \%$ & $92.3 \%$ \\
\hline 0.85 & $2.6 \%$ & $0.3 \%$ & $0.2 \%$ & $0 \%$ & $6.5 \%$ & $19.7 \%$ & $36.5 \%$ & $73.2 \%$ \\
\hline 0.9 & $3.2 \%$ & $0.7 \%$ & $0.5 \%$ & $0.1 \%$ & $5.7 \%$ & $10.4 \%$ & $19.7 \%$ & $39.9 \%$ \\
\hline 0.95 & $5.1 \%$ & $2 \%$ & $1.5 \%$ & $0.6 \%$ & $5.3 \%$ & $5.1 \%$ & $8.5 \%$ & $13.6 \%$ \\
\hline 1 & $6.9 \%$ & $5 \%$ & $5.2 \%$ & $5.5 \%$ & $5.6 \%$ & $4.1 \%$ & $5.2 \%$ & $5 \%$ \\
\hline 1.05 & $10.1 \%$ & $10.7 \%$ & $14 \%$ & $22.9 \%$ & $6.5 \%$ & $6.4 \%$ & $9.4 \%$ & $15.3 \%$ \\
\hline 1.1 & $14 \%$ & $21.5 \%$ & $29.9 \%$ & $54.1 \%$ & $8.4 \%$ & $12.3 \%$ & $20.8 \%$ & $43.2 \%$ \\
\hline 1.15 & $17.7 \%$ & $33.9 \%$ & $50.7 \%$ & $82.8 \%$ & $11.2 \%$ & $24 \%$ & $37.1 \%$ & $74.4 \%$ \\
\hline 1.2 & $21.5 \%$ & $47.1 \%$ & $70.7 \%$ & $97.1 \%$ & $14.9 \%$ & $35.9 \%$ & $58.7 \%$ & $93.3 \%$ \\
\hline 1.25 & $25 \%$ & $62.3 \%$ & $85.6 \%$ & $99.6 \%$ & $19.1 \%$ & $50.4 \%$ & $78.1 \%$ & $99.1 \%$ \\
\hline 1.3 & $30.6 \%$ & $75.2 \%$ & $94.7 \%$ & $100 \%$ & $22.3 \%$ & $64.7 \%$ & $89.9 \%$ & $100 \%$ \\
\hline 1.35 & $36.4 \%$ & $86.4 \%$ & $98.1 \%$ & $100 \%$ & $26.2 \%$ & $77.4 \%$ & $96.3 \%$ & $100 \%$ \\
\hline 1.4 & $43.9 \%$ & $93.4 \%$ & $99.6 \%$ & $100 \%$ & $31.7 \%$ & $87.6 \%$ & $98.8 \%$ & $100 \%$ \\
\hline 1.45 & $49.8 \%$ & $97.6 \%$ & $99.9 \%$ & $100 \%$ & $37.4 \%$ & $94 \%$ & $99.7 \%$ & $100 \%$ \\
\hline 1.5 & $57.8 \%$ & $98.8 \%$ & $100 \%$ & $100 \%$ & $45.1 \%$ & $97.9 \%$ & $99.9 \%$ & $100 \%$ \\
\hline 2 & $96.3 \%$ & $100 \%$ & $100 \%$ & $100 \%$ & $93.8 \%$ & $100 \%$ & $100 \%$ & $100 \%$ \\
\hline 2.25 & $99.3 \%$ & $100 \%$ & $100 \%$ & $100 \%$ & $98.6 \%$ & $100 \%$ & $100 \%$ & $100 \%$ \\
\hline 2.5 & $99.9 \%$ & $100 \%$ & $100 \%$ & $100 \%$ & $99.7 \%$ & $100 \%$ & $100 \%$ & $100 \%$ \\
\hline 2.75 & $100 \%$ & $100 \%$ & $100 \%$ & $100 \%$ & $99.9 \%$ & $100 \%$ & $100 \%$ & $100 \%$ \\
\hline 3 & $100 \%$ & $100 \%$ & $100 \%$ & $100 \%$ & $100 \%$ & $100 \%$ & $100 \%$ & $100 \%$ \\
\hline
\end{tabular}

The point tested is $\beta^{r}=\frac{r}{\sqrt{2} \Pi}\left[\begin{array}{l}1 \\ 0\end{array}\right] . \beta^{1}$ is on the frontier of $\mathrm{B}$. 
Table 3: Results related to the Monte Carlo simulations - example 2 with supernumerary instruments

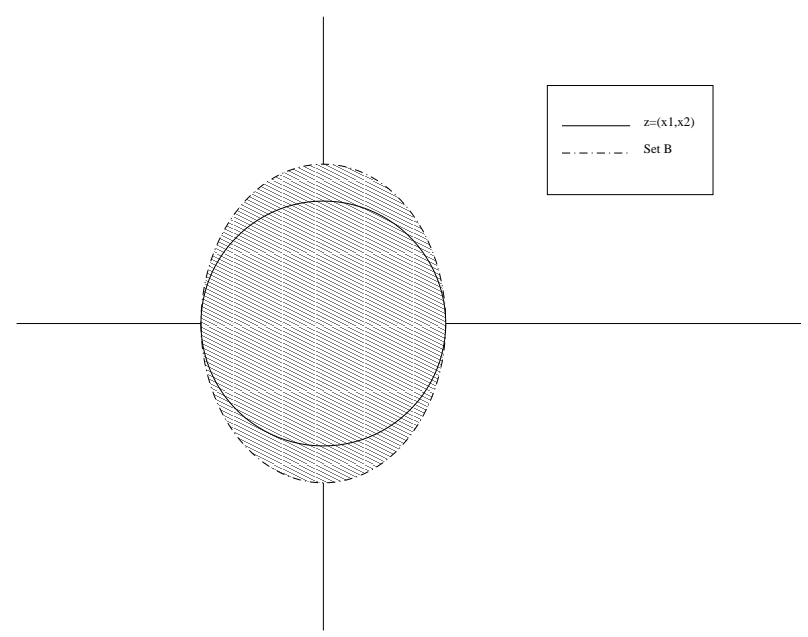

Set B, $y=0 . x_{1}+0 . x_{2}+\varepsilon, z=\left(x_{1}, e_{2}, w\right)$

Support function $\delta(q)$ for $q=(0,1)^{T}$

True unknown value: 0.243

\begin{tabular}{ccccc}
\hline \hline $\mathrm{n}$ & Mean & $\mathrm{Q} 1$ & $\mathrm{Q} 2$ & $\mathrm{Q} 3$ \\
\hline 100 & 0.244 & 0.216 & 0.242 & 0.268 \\
500 & 0.244 & 0.232 & 0.244 & 0.256 \\
1000 & 0.243 & 0.234 & 0.243 & 0.252 \\
2500 & 0.243 & 0.238 & 0.243 & 0.248 \\
\hline \hline
\end{tabular}


Table 4: Percentage of rejections for the two tests - second example

\begin{tabular}{|c|c|c|c|c|c|c|c|c|}
\hline \multirow{3}{*}{$\begin{array}{l}r \\
0\end{array}$} & \multicolumn{4}{|c|}{$\begin{array}{c}\text { Test } 1 \\
\left(H_{0}: \beta^{r} \in B\right)\end{array}$} & \multicolumn{4}{|c|}{$\begin{array}{c}\text { Test } 2 \\
\left(H_{0}: \beta^{r} \in \partial B\right)\end{array}$} \\
\hline & $n=100$ & $n=500$ & $n=1000$ & $n=2500$ & $n=100$ & $n=500$ & $n=1000$ & $n=2500$ \\
\hline & $0 \%$ & $0 \%$ & $0 \%$ & $0 \%$ & $62.1 \%$ & $100 \%$ & $100 \%$ & $100 \%$ \\
\hline 0.05 & $0 \%$ & $0 \%$ & $0 \%$ & $0 \%$ & $62 \%$ & $100 \%$ & $100 \%$ & $100 \%$ \\
\hline 0.1 & $0 \%$ & $0 \%$ & $0 \%$ & $0 \%$ & $59.7 \%$ & $100 \%$ & $100 \%$ & $100 \%$ \\
\hline 0.2 & $0 \%$ & $0 \%$ & $0 \%$ & $0 \%$ & $54 \%$ & $100 \%$ & $100 \%$ & $100 \%$ \\
\hline 0.3 & $0 \%$ & $0 \%$ & $0 \%$ & $0 \%$ & $45.6 \%$ & $100 \%$ & $100 \%$ & $100 \%$ \\
\hline 0.4 & $0 \%$ & $0 \%$ & $0 \%$ & $0 \%$ & $34.4 \%$ & $99.5 \%$ & $100 \%$ & $100 \%$ \\
\hline 0.5 & $0.1 \%$ & $0 \%$ & $0 \%$ & $0 \%$ & $23.2 \%$ & $96.4 \%$ & $99.9 \%$ & $100 \%$ \\
\hline 0.6 & $0.4 \%$ & $0 \%$ & $0 \%$ & $0 \%$ & $15.7 \%$ & $83.5 \%$ & $99.3 \%$ & $100 \%$ \\
\hline 0.7 & $1 \%$ & $0 \%$ & $0 \%$ & $0 \%$ & $9.7 \%$ & $59.1 \%$ & $87.8 \%$ & $99.8 \%$ \\
\hline 0.8 & $2.8 \%$ & $0 \%$ & $0 \%$ & $0 \%$ & $6.4 \%$ & $28 \%$ & $52.1 \%$ & $90.7 \%$ \\
\hline 0.85 & $3.6 \%$ & $0.3 \%$ & $0.1 \%$ & $0 \%$ & $5.7 \%$ & $15.6 \%$ & $32.9 \%$ & $70 \%$ \\
\hline 0.9 & $4.6 \%$ & $0.9 \%$ & $0.5 \%$ & $0.1 \%$ & $5.4 \%$ & $8.9 \%$ & $15.4 \%$ & $33.7 \%$ \\
\hline 0.92 & $5.2 \%$ & $1.5 \%$ & $0.7 \%$ & $0.2 \%$ & $5.3 \%$ & $6.3 \%$ & $9.5 \%$ & $23 \%$ \\
\hline 0.94 & $5.4 \%$ & $2.1 \%$ & $1 \%$ & $0.8 \%$ & $5.6 \%$ & $5 \%$ & $6.1 \%$ & $14.6 \%$ \\
\hline 0.96 & $5.6 \%$ & $2.8 \%$ & $2 \%$ & $1.3 \%$ & $5.5 \%$ & $4.7 \%$ & $4.6 \%$ & $7.8 \%$ \\
\hline 0.98 & $6.8 \%$ & $3.5 \%$ & $3.2 \%$ & $3.4 \%$ & $5.9 \%$ & $4.4 \%$ & $4.4 \%$ & $4.8 \%$ \\
\hline 0.99 & $7.1 \%$ & $4.4 \%$ & $4.4 \%$ & $4.1 \%$ & $5.8 \%$ & $4.4 \%$ & $4.4 \%$ & $5 \%$ \\
\hline 1 & $7.9 \%$ & $5.4 \%$ & $5.9 \%$ & $5.5 \%$ & $6.1 \%$ & $4.8 \%$ & $3.9 \%$ & $5.2 \%$ \\
\hline 1.01 & $8.3 \%$ & $6.3 \%$ & $7.2 \%$ & $8.5 \%$ & $6.3 \%$ & $4.8 \%$ & $4.7 \%$ & $5.6 \%$ \\
\hline 1.02 & $8.5 \%$ & $7.3 \%$ & $8.4 \%$ & $11.5 \%$ & $6.4 \%$ & $5 \%$ & $5.8 \%$ & $6.7 \%$ \\
\hline 1.04 & $9.7 \%$ & $9.7 \%$ & $12.1 \%$ & $18.7 \%$ & $6.6 \%$ & $6 \%$ & $8 \%$ & $12.4 \%$ \\
\hline 1.06 & $10.2 \%$ & $12.9 \%$ & $16.6 \%$ & $28.5 \%$ & $7.3 \%$ & $7.6 \%$ & $10.1 \%$ & $19.5 \%$ \\
\hline 1.08 & $11.3 \%$ & $17.4 \%$ & $22.4 \%$ & $40.4 \%$ & $7.9 \%$ & $9.9 \%$ & $14.3 \%$ & $28.9 \%$ \\
\hline 1.1 & $12.3 \%$ & $20.3 \%$ & $29.3 \%$ & $55.8 \%$ & $8.5 \%$ & $12.7 \%$ & $20.1 \%$ & $41.4 \%$ \\
\hline 1.2 & $21.6 \%$ & $47.5 \%$ & $70.6 \%$ & $97.3 \%$ & $13.8 \%$ & $35.2 \%$ & $58.6 \%$ & $94.3 \%$ \\
\hline 1.3 & $33.6 \%$ & $75.3 \%$ & $95.9 \%$ & $100 \%$ & $22.9 \%$ & $64.9 \%$ & $92.2 \%$ & $100 \%$ \\
\hline 1.4 & $46.1 \%$ & $93.3 \%$ & $99.5 \%$ & $100 \%$ & $34.7 \%$ & $87.5 \%$ & $98.8 \%$ & $100 \%$ \\
\hline 1.5 & $60.9 \%$ & $98.3 \%$ & $100 \%$ & $100 \%$ & $47 \%$ & $97.2 \%$ & $100 \%$ & $100 \%$ \\
\hline 1.6 & $69.6 \%$ & $99.9 \%$ & $100 \%$ & $100 \%$ & $60.9 \%$ & $99.6 \%$ & $100 \%$ & $100 \%$ \\
\hline 1.8 & $88.5 \%$ & $100 \%$ & $100 \%$ & $100 \%$ & $81.5 \%$ & $100 \%$ & $100 \%$ & $100 \%$ \\
\hline 2.05 & $97.9 \%$ & $100 \%$ & $100 \%$ & $100 \%$ & $94.8 \%$ & $100 \%$ & $100 \%$ & $100 \%$ \\
\hline 2.3 & $99.8 \%$ & $100 \%$ & $100 \%$ & $100 \%$ & $99.3 \%$ & $100 \%$ & $100 \%$ & $100 \%$ \\
\hline 2.55 & $100 \%$ & $100 \%$ & $100 \%$ & $100 \%$ & $100 \%$ & $100 \%$ & $100 \%$ & $100 \%$ \\
\hline 2.8 & $100 \%$ & $100 \%$ & $100 \%$ & $100 \%$ & $100 \%$ & $100 \%$ & $100 \%$ & $100 \%$ \\
\hline
\end{tabular}


Table 5: Percentage of rejections for the test $H_{0}: \beta^{r} \in \partial B$. Non-smooth set.

\begin{tabular}{|c|c|c|c|c|c|c|c|c|}
\hline \multirow[b]{2}{*}{$r$} & \multicolumn{4}{|c|}{ Test with $a_{n}=0$} & \multicolumn{4}{|c|}{ Test with $a_{n}=\frac{0.5}{n^{1 / 3}}$} \\
\hline & $n=100$ & $n=500$ & $n=1000$ & $n=2500$ & $n=100$ & $n=500$ & $n=1000$ & $n=2500$ \\
\hline 0.010 & $100 \%$ & $100 \%$ & $100 \%$ & $100 \%$ & $100 \%$ & $100 \%$ & $100 \%$ & $100 \%$ \\
\hline 0.050 & $100 \%$ & $100 \%$ & $100 \%$ & $100 \%$ & $100 \%$ & $100 \%$ & $100 \%$ & $100 \%$ \\
\hline 0.100 & $99.8 \%$ & $100 \%$ & $100 \%$ & $100 \%$ & $100 \%$ & $100 \%$ & $100 \%$ & $100 \%$ \\
\hline 0.200 & $100 \%$ & $100 \%$ & $100 \%$ & $100 \%$ & $100 \%$ & $100 \%$ & $100 \%$ & $100 \%$ \\
\hline 0.300 & $99.8 \%$ & $100 \%$ & $100 \%$ & $100 \%$ & $99.8 \%$ & $100 \%$ & $100 \%$ & $100 \%$ \\
\hline 0.400 & $98.5 \%$ & $100 \%$ & $100 \%$ & $100 \%$ & $98.5 \%$ & $100 \%$ & $100 \%$ & $100 \%$ \\
\hline 0.500 & $94.1 \%$ & $100 \%$ & $100 \%$ & $100 \%$ & $95 \%$ & $100 \%$ & $100 \%$ & $100 \%$ \\
\hline 0.600 & $78.2 \%$ & $100 \%$ & $100 \%$ & $100 \%$ & $84 \%$ & $100 \%$ & $100 \%$ & $100 \%$ \\
\hline 0.700 & $41.9 \%$ & $99.8 \%$ & $100 \%$ & $100 \%$ & $61.3 \%$ & $99.8 \%$ & $100 \%$ & $100 \%$ \\
\hline 0.800 & $9.9 \%$ & $90.9 \%$ & $100 \%$ & $100 \%$ & $29.1 \%$ & $96.2 \%$ & $100 \%$ & $100 \%$ \\
\hline 0.850 & $4.2 \%$ & $60.6 \%$ & $94.5 \%$ & $100 \%$ & $15.4 \%$ & $85.7 \%$ & $99.2 \%$ & $100 \%$ \\
\hline 0.900 & $1.8 \%$ & $20 \%$ & $52.6 \%$ & $97.7 \%$ & $7.6 \%$ & $55.3 \%$ & $87.3 \%$ & $99.9 \%$ \\
\hline 0.910 & $1.6 \%$ & $14.7 \%$ & $40.4 \%$ & $92.9 \%$ & $6.4 \%$ & $48.3 \%$ & $79.6 \%$ & $99.8 \%$ \\
\hline 0.920 & $1.8 \%$ & $9 \%$ & $28.5 \%$ & $84.2 \%$ & $5.8 \%$ & $40.1 \%$ & $70.2 \%$ & $98.8 \%$ \\
\hline 0.930 & $2.3 \%$ & $5.6 \%$ & $18.8 \%$ & $66.4 \%$ & $4.8 \%$ & $30.8 \%$ & $59.8 \%$ & $96.2 \%$ \\
\hline 0.940 & $2.3 \%$ & $3.4 \%$ & $11 \%$ & $44.1 \%$ & $4.3 \%$ & $23.6 \%$ & $47 \%$ & $90.1 \%$ \\
\hline 0.950 & $2.7 \%$ & $2.1 \%$ & $6.6 \%$ & $25.4 \%$ & $3.9 \%$ & $17 \%$ & $33.5 \%$ & $76.7 \%$ \\
\hline 0.960 & $2.9 \%$ & $1.7 \%$ & $3.4 \%$ & $13.1 \%$ & $3.7 \%$ & $11.9 \%$ & $20.9 \%$ & $54.4 \%$ \\
\hline 0.970 & $3.3 \%$ & $1.6 \%$ & $2.8 \%$ & $5.5 \%$ & $3.8 \%$ & $7.9 \%$ & $12 \%$ & $32.1 \%$ \\
\hline 0.980 & $3.8 \%$ & $2.4 \%$ & $3.4 \%$ & $2.4 \%$ & $3.8 \%$ & $4.9 \%$ & $8 \%$ & $14.9 \%$ \\
\hline 0.990 & $4.9 \%$ & $3.9 \%$ & $3.9 \%$ & $1.9 \%$ & $4.7 \%$ & $4.2 \%$ & $5.3 \%$ & $6.2 \%$ \\
\hline $1 \%$ & $6.4 \%$ & $6.5 \%$ & $6.4 \%$ & $4.7 \%$ & $5.3 \%$ & $5.2 \%$ & $5.2 \%$ & $4.9 \%$ \\
\hline 1.010 & $7.9 \%$ & $9.3 \%$ & $11.7 \%$ & $12.3 \%$ & $5.7 \%$ & $7.3 \%$ & $9.2 \%$ & $9.2 \%$ \\
\hline 1.020 & $9 \%$ & $13.1 \%$ & $19.7 \%$ & $30.4 \%$ & $6.4 \%$ & $9.9 \%$ & $15.8 \%$ & $22.6 \%$ \\
\hline 1.030 & $10.1 \%$ & $16.9 \%$ & $29.4 \%$ & $53.2 \%$ & $7.9 \%$ & $13.6 \%$ & $23.6 \%$ & $44.4 \%$ \\
\hline 1.040 & $12.3 \%$ & $25.6 \%$ & $41 \%$ & $73.5 \%$ & $9.8 \%$ & $19.1 \%$ & $34.8 \%$ & $67.4 \%$ \\
\hline 1.050 & $14.1 \%$ & $33.7 \%$ & $54.3 \%$ & $88.2 \%$ & $11.9 \%$ & $27.8 \%$ & $48.6 \%$ & $86.4 \%$ \\
\hline 1.060 & $16.4 \%$ & $44 \%$ & $67.4 \%$ & $95.7 \%$ & $13.6 \%$ & $37.8 \%$ & $62.5 \%$ & $94.7 \%$ \\
\hline 1.070 & $19.3 \%$ & $52.9 \%$ & $80.3 \%$ & $98.9 \%$ & $15.7 \%$ & $46.5 \%$ & $76.7 \%$ & $98.7 \%$ \\
\hline 1.080 & $21.8 \%$ & $63.3 \%$ & $88.7 \%$ & $99.5 \%$ & $18.1 \%$ & $59.1 \%$ & $86.8 \%$ & $99.4 \%$ \\
\hline 1.090 & $24.2 \%$ & $71 \%$ & $93.9 \%$ & $99.9 \%$ & $21.2 \%$ & $67.7 \%$ & $92.2 \%$ & $99.8 \%$ \\
\hline 1.100 & $27.7 \%$ & $79.2 \%$ & $97 \%$ & $100 \%$ & $23.6 \%$ & $76.6 \%$ & $96 \%$ & $100 \%$ \\
\hline 1.150 & $48.4 \%$ & $98.5 \%$ & $100 \%$ & $100 \%$ & $44.6 \%$ & $98.3 \%$ & $100 \%$ & $100 \%$ \\
\hline 1.200 & $68.6 \%$ & $100 \%$ & $100 \%$ & $100 \%$ & $66 \%$ & $100 \%$ & $100 \%$ & $100 \%$ \\
\hline 1.300 & $95.1 \%$ & $100 \%$ & $100 \%$ & $100 \%$ & $94.7 \%$ & $100 \%$ & $100 \%$ & $100 \%$ \\
\hline 1.400 & $99.9 \%$ & $100 \%$ & $100 \%$ & $100 \%$ & $99.8 \%$ & $100 \%$ & $100 \%$ & $100 \%$ \\
\hline 1.500 & $100 \%$ & $100 \%$ & $100 \%$ & $100 \%$ & $100 \%$ & $100 \%$ & $100 \%$ & $100 \%$ \\
\hline 1.600 & $100 \%$ & $100 \%$ & $100 \%$ & $100 \%$ & $100 \%$ & $100 \%$ & $100 \%$ & $100 \%$ \\
\hline 1.700 & $100 \%$ & $100 \%$ & $100 \%$ & $100 \%$ & $100 \%$ & $100 \%$ & $100 \%$ & $100 \%$ \\
\hline 1.800 & $100 \%$ & $100 \%$ & $100 \%$ & $100 \%$ & $100 \%$ & $100 \%$ & $100 \%$ & $100 \%$ \\
\hline 1.900 & $100 \%$ & $100 \%$ & $100 \%$ & $100 \%$ & $100 \%$ & $100 \%$ & $100 \%$ & $100 \%$ \\
\hline 2.000 & $100 \%$ & $100 \%$ & $100 \%$ & $100 \%$ & $100 \%$ & $100 \%$ & $100 \%$ & $100 \%$ \\
\hline
\end{tabular}




\section{E Additional Appendix}

\section{E.1 Proof of Proposition 8}

We denote $M$ a generic majorizing constant. The estimate of the support function is:

$$
\frac{1}{n} \sum_{i=1}^{n} z_{n, q i} w_{n, q i}=\frac{1}{n} \sum_{i=1}^{n} f_{\hat{\theta}_{n}}\left(z_{i}, \underline{y}_{i}, \bar{y}_{i}\right)
$$

where $\hat{\theta}_{n}=\left(q, \hat{\Sigma}_{n}\right)$. First, under the conditions of Proposition 8, the class $\mathcal{F}=\left\{f_{\theta} ; \theta \in \Theta\right\}$ is a Glivenko-Cantelli class. By construction of the estimate $\hat{\Sigma}_{n}$ (see Appendix C.1 above), $\hat{\theta}_{n}$ belongs to $\Theta$. It is thus immediate that, for every sequence of functions $f_{\hat{\theta}_{n}} \in \mathcal{F}$, and uniformly in $q \in \mathbb{S}$, we have:

$$
\left|\frac{1}{n} \sum_{i=1}^{n} f_{\hat{\theta}_{n}}\left(z_{i}, \underline{y}_{i}, \bar{y}_{i}\right)-E\left(f_{\hat{\theta}_{n}}\left(z_{i}, \underline{y}_{i}, \bar{y}_{i}\right)\right)\right| \underset{n \rightarrow \infty}{\stackrel{a . s}{\longrightarrow}} 0 .
$$

Second, as matrix $\Sigma$ is estimated by its almost surely consistent empirical analogue $\hat{\Sigma}_{n}$ :

$$
\lim _{n \rightarrow \infty} \operatorname{Pr}\left(\sup _{n>N}\left\|\hat{\Sigma}_{n}-\Sigma\right\| \geq \varepsilon\right)=0,
$$

we have:

$$
\lim _{n \rightarrow \infty} \operatorname{Pr}\left(\sup _{n>N} \sup _{q \in \mathbb{S}}\left\|\hat{\theta}_{n}-\theta\right\| \geq \varepsilon\right)=0
$$

Use equation (C.11):

$$
\left.\left|f_{\hat{\theta}_{n}}\left(z_{i}, \underline{y}_{i}, \bar{y}_{i}\right)-f_{\theta}\left(z_{i}, \underline{y}_{i}, \bar{y}_{i}\right)\right|=\left|z_{n, q i} w_{n, q i}-z_{q i} w_{q i}\right| \leq \max \left(\left\|z_{i}^{\top} \underline{y}_{i}\right\|, \| z_{i}^{\top} \bar{y}_{i}\right) \|\right) M\left\|\hat{\theta}_{n}-\theta\right\|
$$

to conclude that, uniformly over $q \in \mathbb{S}$, we have:

$$
\left|f_{\hat{\theta}_{n}}\left(z_{i}, \underline{y}_{i}, \bar{y}_{i}\right)-f_{\theta}\left(z_{i}, \underline{y}_{i}, \bar{y}_{i}\right)\right| \underset{n \rightarrow \infty}{\stackrel{a . s}{\rightarrow}} 0 .
$$

To finish the proof, notice that the sequence $f_{\hat{\theta}_{n}}\left(z_{i}, \underline{y}_{i}, \bar{y}_{i}\right)$ is uniformly bounded for $q \in \mathbb{S}$, because, by majorization and triangular inequality, we have:

$$
f_{\hat{\theta}_{n}}\left(z_{i}, \underline{y}_{i}, \bar{y}_{i}\right)=\left|z_{n, q i} w_{n, q i}\right| \leq\left\|q^{\top} \Sigma_{n}^{\top}\right\|\left(\left\|z_{i}^{\top} \bar{y}_{i}\right\|+\left\|z_{i}^{\top} \underline{y}_{i}\right\|\right)=\left\|\Sigma_{n}\right\|\left(\left\|z_{i}^{\top} \bar{y}_{i}\right\|+\left\|z_{i}^{\top} \underline{y}_{i}\right\|\right)
$$

since $\|q\|=1$. Therefore, as $\left\|\Sigma_{n}\right\| \leq M$ :

$$
\sup _{q \in \mathbb{S}}\left|f_{\hat{\theta}_{n}}\left(z_{i}, \underline{y}_{i}, \bar{y}_{i}\right)\right| \leq M\left(\left\|z_{i}^{\top} \bar{y}_{i}\right\|+\left\|z_{i}^{\top} \underline{y}_{i}\right\|\right)
$$

As $z_{i}, \bar{y}, \underline{y}_{i}$ are in $L^{2}$ (Assumption R.iii), it implies that:

$$
E \sup _{q \in \mathbb{S}}\left|f_{\hat{\theta}_{n}}\left(z_{i}, \underline{y}_{i}, \bar{y}_{i}\right)\right| \leq M<+\infty .
$$

Thus, equation (E.30) implies that, by the dominated convergence theorem, uniformly over $q$,

$$
E\left|f_{\hat{\theta}_{n}}\left(z_{i}, \underline{y}_{i}, \bar{y}_{i}\right)-f_{\theta}\left(z_{i}, \underline{y}_{i}, \bar{y}_{i}\right)\right| \underset{n \rightarrow \infty}{\stackrel{a . s}{\rightarrow}} 0 .
$$

From the latter equation, equation (E.29) and the triangular inequality, we thus conclude that, uniformly for $q \in \mathbb{S}$ :

$$
\frac{1}{n} \sum_{i=1}^{n} z_{n, q i} w_{n, q i} \underset{n \rightarrow \infty}{\stackrel{a . s}{\longrightarrow}} E\left(z_{q i} w_{q i}\right) .
$$




\section{E.2 Construction of the Confidence Region in Proposition 11}

Like before, for the simplicity of the exposition, we focus on the case where $B$ is strictly convex. We here provide a simple way to construct $C I_{\alpha}^{n}$ when $\alpha<1 / 2$ :

$$
C I_{\alpha}^{n}=\left\{\beta ; \frac{\sqrt{n}}{\sqrt{\hat{V}_{q_{n}}}}\left(T_{n}\left(q_{n} ; \beta\right)\right)>\mathcal{N}_{\alpha}\right\}
$$

where

$$
T_{n}(q ; \beta)=\left(\hat{\delta}_{n}^{*}(q \mid B)-q^{\top} \beta\right),
$$

and where $q_{n}$ is one argument of the minimum of $T_{n}(q ; \beta)$ over the unit sphere (and therefore depends on $\beta$ ). Therefore, the confidence region is also given by $C I_{\alpha}^{n}=\left\{\beta ; \min _{q \in \mathbb{S}}\left(T_{n}(q ; \beta)\right)>\right.$ $\left.\frac{\sqrt{\hat{V}_{q_{n}}}}{\sqrt{n}} \mathcal{N}_{\alpha}\right\}$

The estimated set $\hat{B}_{n}$ is included in $C I_{\alpha}^{n}$ as $\mathcal{N}_{\alpha}<0$ for any $\alpha<1 / 2$ and as for all $\beta$ belonging to the the estimated set, $\hat{B}_{n}$ :

$$
\min _{q \in \mathbb{S}}\left(\hat{\delta}_{n}^{*}(q \mid B)-q^{\top} \beta\right) \geq 0
$$

Consider any point $\beta_{f} \in \partial \hat{B}_{n} \subset C I_{\alpha}^{n}$, the frontier of the estimated set $\hat{B}_{n}$. There exists at least one, and possibly a set (which is the intersection of a cone and $\mathbb{S}$ ) denoted $\mathcal{C}\left(\beta_{f}\right)$, of vectors $q_{f} \in \mathbb{S}$ such that:

$$
\begin{aligned}
& T_{n}\left(q_{f} ; \beta_{f}\right)=\hat{\delta}_{n}^{*}\left(q_{f} \mid B\right)-q_{f}^{\top} \beta_{f}=0, \\
& \quad \forall q \in \mathbb{S}, T_{n}\left(q ; \beta_{f}\right) \geq T_{n}\left(q_{f} ; \beta_{f}\right)=0
\end{aligned}
$$

Choose such a $q_{f}$ and consider the points $\beta_{f}(\lambda)$, where $\lambda \geq 0$, on the half-line defined by $\beta_{f}$ and direction $q_{f}$ :

$$
\beta_{f}(\lambda)=\beta_{f}+\lambda q_{f}
$$

We have:

$$
\begin{aligned}
T_{n}\left(q ; \beta_{f}(\lambda)\right) & =T_{n}\left(q ; \beta_{f}\right)+q^{\top}\left(\beta_{f}-\beta_{f}(\lambda)\right) \\
& =T_{n}\left(q ; \beta_{f}\right)-\lambda q^{\top} q_{f}
\end{aligned}
$$

where $-\lambda q^{\top} q_{f} \geq-\lambda q_{f}^{\top} q_{f}=-\lambda$ and $T_{n}\left(q ; \beta_{f}\right) \geq T_{n}\left(q_{f} ; \beta_{f}\right)=0$ for any $q$, as seen above. As a consequence,

$$
T_{n}\left(q ; \beta_{f}(\lambda)\right) \geq-\lambda=T_{n}\left(q_{f} ; \beta_{f}(\lambda)\right) .
$$

where vector $q_{f}$ which minimizes $T_{n}\left(q ; \beta_{f}\right)$ minimizes also $T_{n}\left(q ; \beta_{f}(\lambda)\right)$.

We can therefore characterize the points of the half-line which belongs to $C I_{\alpha}^{n}$. Given that $\lambda$ is positive,

$$
\beta_{f}(\lambda) \in C I_{\alpha}^{n} \text { if and only if } \lambda \leq-\frac{\sqrt{\hat{V}_{q_{f}}}}{\sqrt{n}} \mathcal{N}_{\alpha},
$$

so that segment $\left(\beta_{f}, \beta_{f}-\frac{\sqrt{\hat{V}_{f}}}{\sqrt{n}} \mathcal{N}_{\alpha} q_{f}\right]$ is included in $C I_{\alpha}^{n}$. We thus proved that:

$$
\hat{B}_{n} \cup\left\{\cup_{\beta_{f} \in \partial B_{n}} \cup_{q_{f} \in \mathcal{C}\left(\beta_{f}\right)}\left(\beta_{f}, \beta_{f}-\frac{\sqrt{\hat{V}_{q_{f}}}}{\sqrt{n}} \mathcal{N}_{\alpha} q_{f}\right)\right\} \subset C I_{\alpha}^{n},
$$


where $\mathcal{C}\left(\beta_{f}\right)$ is the cone defined above.

Conversely, let us prove that $C I_{\alpha}^{n}$ is included in the set on the LHS. Let $\beta_{c}$ a point in $C I_{\alpha}^{n}$. If $\beta_{c}$ belongs to $\hat{B}_{n}$, the inclusion is proved. Assume that $\beta_{c}$ is outside the estimated set and let $\beta_{f}$ the point on the frontier of $\hat{B}_{n}$ which is the projection of $\beta_{c}$ on $\hat{B}_{n}$. The projection is unique because set $\hat{B}$ is convex.

Write $\beta_{c}-\beta_{f}=\lambda q_{f}$ for some direction $q_{f} \in \mathbb{S}$ and some $\lambda>0$. We have that:

$$
q_{f}^{\top}\left(\beta_{c}-\beta_{f}\right) \leq q_{f}^{\top}\left(\beta_{c}-\beta\right),
$$

for any $\beta \in \hat{B}_{n}$ because $\beta_{f}$ is the projection of $\beta_{c}$ on set $\hat{B}_{n}$ along the direction $q_{f}$. We thus have $q_{f}^{\top} \beta_{f} \geq q_{f}^{\top} \beta$ which proves that $\hat{\delta}_{n}^{*}\left(q_{f} \mid B\right)=q_{f}^{\top} \beta_{f}$. The pair $\left(\beta_{f}, q_{f}\right)$ satisfies the condition of the previous paragraphs.

As $\beta_{c}$ is a point of $C I_{\alpha}^{n}, \lambda$ is necessary less or equal than the value $-\frac{\sqrt{\hat{V}_{q_{f}}}}{\sqrt{n}} \mathcal{N}_{\alpha}$. Thus it belongs to the LHS of equation (E.31). As a consequence, equation (E.31) is an equality.

\section{E.3 Behaviour of $\xi_{n}(\beta)$ when the set is a singleton}

When $B=\left\{\beta_{0}\right\}$, it means that $w_{q}$ is constant, equal to $y_{e}$ (either $\bar{y}$ or $\underline{y}$ ). Consequently, $\beta_{0}=$ $E\left(z^{\top} x\right)^{-1} E\left(z^{\top} y_{e}\right)$. Let $\beta_{n}$ be the point where the previous expectations are replaced by their empirical counterpart: $\hat{\delta}_{n}^{*}=q^{\top} \beta_{n}$. A CLT can therefore be applied to $\beta_{n}$ :

$$
\sqrt{n}\left(\beta_{n}-\beta_{0}\right) \underset{n \rightarrow+\infty}{\longrightarrow} N(0, V),
$$

where $V$ is some p.d matrix.

If we test a point $\beta \neq \beta_{0}, \xi_{n}(\beta)$ tends to $-\infty\left(q_{0}\right.$ is in this case $\left.\frac{\beta-\beta_{0}}{\left\|\beta-\beta_{0}\right\|}\right)$.

When $\beta=\beta_{0}$,

$$
\begin{aligned}
T_{n}\left(q ; \beta_{0}\right) & =\left(\hat{\delta}_{n}(q)-q^{\top} \beta_{0}\right) \\
& =q^{\top}\left(\beta_{n}-\beta_{0}\right)
\end{aligned}
$$

In this case $q_{n}=-\frac{\beta_{n}-\beta_{0}}{\left\|\beta_{n}-\beta_{0}\right\|}$ and $T_{n}\left(q_{n} ; \beta_{0}\right)=-\left\|\beta_{n}-\beta_{0}\right\|$.

And, after standardization:

$$
\xi_{n}\left(\beta_{0}\right)=-\|u\|,
$$

where $u$ tends asymptotically toward a standard normal distribution. If we use the usual critical values to construct the confidence region, i.e. $\mathcal{N}_{\alpha}$, the probability that $\xi_{n}\left(\beta_{0}\right)$ is greater than this value is not $1-\alpha$ but $1-2 \alpha$.

\section{E.4 Uniform confidence regions}

The empirical counterpart $\hat{\Delta}_{n}$ of the diameter of the set B is:

$$
\hat{\Delta}_{n}=\max _{q \in \mathbb{S}}\left(\hat{\delta}_{n}^{*}(q \mid B)+\hat{\delta}_{n}^{*}(-q \mid B)\right) .
$$

Using a proof analogue to the one developed in Proposition 10:

$$
\sqrt{n}\left(\hat{\Delta}_{n}-\Delta\right) \underset{n \rightarrow \infty}{\stackrel{P}{\longrightarrow}} 0 .
$$

The next proposition provides an extension of Lemma 4 of Imbens and Manski (2004) in the multivariate case for constructing a uniform confidence region: 


\section{Proposition 19 Let}

$$
\hat{\sigma}_{n}=\sqrt{\hat{V}_{q_{n}}}=\sqrt{q_{n}^{\top} \hat{\Sigma}_{n} \hat{V}\left(z^{\top} \varepsilon_{q_{n}}\right) \hat{\Sigma}_{n} q_{n}}
$$

where $q_{n}$ is the argument of the maximum of equation (E.32).

A confidence region $\tilde{C} I_{\alpha}^{n}$ of asymptotic level equal to $1-\alpha$ is defined by the collection of the points such that $\xi(\beta) \geq \tilde{\mathcal{N}}_{\alpha}^{\alpha}$ where $\tilde{\mathcal{N}}_{\alpha}$ satisfies the equation

$$
\begin{gathered}
\Phi\left(\tilde{\mathcal{N}}_{\alpha}+\sqrt{n} \frac{\hat{\Delta}_{n}}{\hat{\sigma}_{n}}\right)-\Phi\left(-\tilde{\mathcal{N}}_{\alpha}\right)=\alpha . \\
\lim _{n \rightarrow+\infty} \inf _{\beta \in B, \Delta \geq 0} \operatorname{Pr}\left(\beta \in \tilde{C} I_{\alpha}^{n}\right)=1-\alpha .
\end{gathered}
$$




\section{E.5 Supplementary Tables}

Table 6: Results related to the Monte Carlo simulations - nonsmooth set

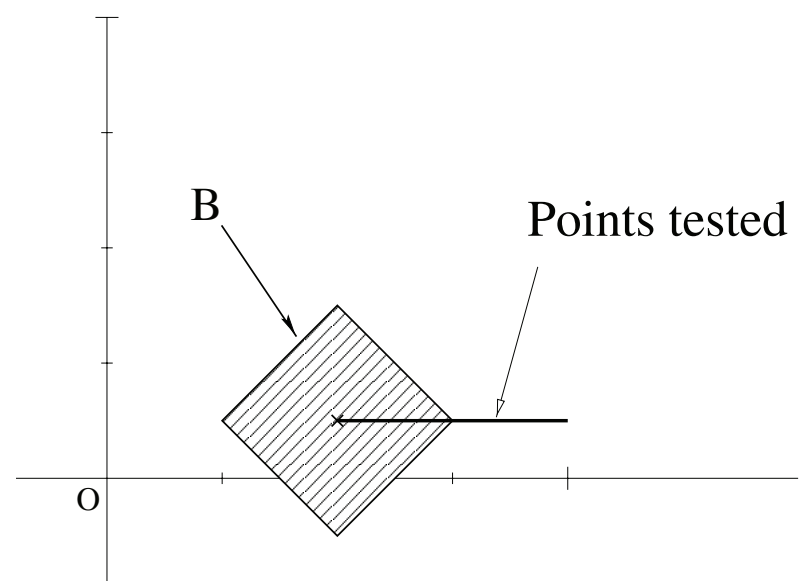

Set B, $y=\frac{1}{2}+\frac{x}{8}+\varepsilon, x \in\{-1,1\}$

Support function $\delta(q)$ for $q=(0,1)^{T}$

True unknown value: 0.375

\begin{tabular}{ccccc}
\hline \hline $\mathrm{n}$ & Mean & $\mathrm{Q} 1$ & $\mathrm{Q} 2$ & $\mathrm{Q} 3$ \\
\hline 100 & 0.374 & 0.360 & 0.375 & 0.390 \\
500 & 0.375 & 0.369 & 0.375 & 0.382 \\
1000 & 0.375 & 0.371 & 0.375 & 0.380 \\
2500 & 0.375 & 0.372 & 0.375 & 0.378 \\
\hline \hline
\end{tabular}


Table 7: Percentage of rejections for the test $H_{0}: \beta^{r} \in B$. Non-smooth set.

\begin{tabular}{|c|c|c|c|c|c|c|c|c|}
\hline \multirow[b]{2}{*}{$r$} & \multicolumn{4}{|c|}{ Test with $a_{n}=0$} & \multicolumn{4}{|c|}{ Test with $a_{n}=\frac{0.5}{n^{1} / 3}$} \\
\hline & $n=100$ & $n=500$ & $n=1000$ & $n=2500$ & $n=100$ & $n=500$ & $n=1000$ & $n=2500$ \\
\hline 0.010 & $0 \%$ & $0 \%$ & $0 \%$ & $0 \%$ & $0 \%$ & $0 \%$ & $0 \%$ & $0 \%$ \\
\hline 0.050 & $0 \%$ & $0 \%$ & $0 \%$ & $0 \%$ & $0 \%$ & $0 \%$ & $0 \%$ & $0 \%$ \\
\hline 0.100 & $0 \%$ & $0 \%$ & $0 \%$ & $0 \%$ & $0 \%$ & $0 \%$ & $0 \%$ & $0 \%$ \\
\hline 0.200 & $0 \%$ & $0 \%$ & $0 \%$ & $0 \%$ & $0 \%$ & $0 \%$ & $0 \%$ & $0 \%$ \\
\hline 0.300 & $0 \%$ & $0 \%$ & $0 \%$ & $0 \%$ & $0 \%$ & $0 \%$ & $0 \%$ & $0 \%$ \\
\hline 0.400 & $0 \%$ & $0 \%$ & $0 \%$ & $0 \%$ & $0 \%$ & $0 \%$ & $0 \%$ & $0 \%$ \\
\hline 0.500 & $0 \%$ & $0 \%$ & $0 \%$ & $0 \%$ & $0 \%$ & $0 \%$ & $0 \%$ & $0 \%$ \\
\hline 0.600 & $0 \%$ & $0 \%$ & $0 \%$ & $0 \%$ & $0 \%$ & $0 \%$ & $0 \%$ & $0 \%$ \\
\hline 0.700 & $0 \%$ & $0 \%$ & $0 \%$ & $0 \%$ & $0 \%$ & $0 \%$ & $0 \%$ & $0 \%$ \\
\hline 0.800 & $0 \%$ & $0 \%$ & $0 \%$ & $0 \%$ & $0 \%$ & $0 \%$ & $0 \%$ & $0 \%$ \\
\hline 0.850 & $0.5 \%$ & $0 \%$ & $0 \%$ & $0 \%$ & $0.2 \%$ & $0 \%$ & $0 \%$ & $0 \%$ \\
\hline 0.900 & $1.9 \%$ & $0.1 \%$ & $0 \%$ & $0 \%$ & $0.9 \%$ & $0 \%$ & $0 \%$ & $0 \%$ \\
\hline 0.910 & $2.0 \%$ & $0.1 \%$ & $0 \%$ & $0 \%$ & $1.2 \%$ & $0 \%$ & $0 \%$ & $0 \%$ \\
\hline 0.920 & $2.5 \%$ & $0.3 \%$ & $0 \%$ & $0 \%$ & $1.5 \%$ & $0.1 \%$ & $0 \%$ & $0 \%$ \\
\hline 0.930 & $3.1 \%$ & $0.3 \%$ & $0 \%$ & $0 \%$ & $2.0 \%$ & $0.1 \%$ & $0 \%$ & $0 \%$ \\
\hline 0.940 & $3.3 \%$ & $0.4 \%$ & $0 \%$ & $0 \%$ & $2.4 \%$ & $0.1 \%$ & $0 \%$ & $0 \%$ \\
\hline 0.950 & $4.0 \%$ & $1.1 \%$ & $0.2 \%$ & $0 \%$ & $2.9 \%$ & $0.6 \%$ & $0 \%$ & $0 \%$ \\
\hline 0.960 & $5.5 \%$ & $1.8 \%$ & $1.1 \%$ & $0 \%$ & $3.8 \%$ & $0.9 \%$ & $0.1 \%$ & $0 \%$ \\
\hline 0.970 & $6.6 \%$ & $3.3 \%$ & $2.3 \%$ & $0.2 \%$ & $4.8 \%$ & $1.5 \%$ & $0.6 \%$ & $0 \%$ \\
\hline 0.980 & $8.2 \%$ & $5.1 \%$ & $3.6 \%$ & $1.3 \%$ & $5.6 \%$ & $2.6 \%$ & $1.8 \%$ & $0.3 \%$ \\
\hline 0.990 & $9.1 \%$ & $7.4 \%$ & $6.2 \%$ & $3.3 \%$ & $6.3 \%$ & $4.6 \%$ & $3.3 \%$ & $1.4 \%$ \\
\hline 1.000 & $10.8 \%$ & $10.6 \%$ & $11.7 \%$ & $9.3 \%$ & $7.9 \%$ & $7.6 \%$ & $7.4 \%$ & $5.3 \%$ \\
\hline 1.010 & $12.9 \%$ & $14.3 \%$ & $20.3 \%$ & $22.1 \%$ & $9.5 \%$ & $10.8 \%$ & $14.0 \%$ & $15.4 \%$ \\
\hline 1.020 & $14.8 \%$ & $21.4 \%$ & $29.5 \%$ & $44.7 \%$ & $11.5 \%$ & $14.7 \%$ & $22.6 \%$ & $34.3 \%$ \\
\hline 1.030 & $16.9 \%$ & $28.6 \%$ & $40.9 \%$ & $65.9 \%$ & $13.0 \%$ & $20.9 \%$ & $33.7 \%$ & $57.4 \%$ \\
\hline 1.040 & $20.1 \%$ & $38.2 \%$ & $53.9 \%$ & $83.4 \%$ & $15.9 \%$ & $30.5 \%$ & $47.2 \%$ & $79.0 \%$ \\
\hline 1.050 & $22.5 \%$ & $48.8 \%$ & $66.8 \%$ & $93.2 \%$ & $18.7 \%$ & $41.0 \%$ & $60.6 \%$ & $91.6 \%$ \\
\hline 1.060 & $25.6 \%$ & $56.9 \%$ & $79.7 \%$ & $97.4 \%$ & $21.3 \%$ & $49.3 \%$ & $74.7 \%$ & $96.9 \%$ \\
\hline 1.070 & $29.4 \%$ & $65.9 \%$ & $88.2 \%$ & $99.4 \%$ & $23.7 \%$ & $61.0 \%$ & $85.9 \%$ & $98.9 \%$ \\
\hline 1.080 & $33.1 \%$ & $74.7 \%$ & $93.3 \%$ & $99.8 \%$ & $28.0 \%$ & $70 \%$ & $91.6 \%$ & $99.7 \%$ \\
\hline 1.090 & $37.6 \%$ & $81.2 \%$ & $96.8 \%$ & $100 \%$ & $32.5 \%$ & $78.2 \%$ & $95.9 \%$ & $100 \%$ \\
\hline 1.100 & $43.4 \%$ & $87.0 \%$ & $98.5 \%$ & $100 \%$ & $37.3 \%$ & $85.0 \%$ & $98.2 \%$ & $100 \%$ \\
\hline 1.150 & $61.8 \%$ & $99.4 \%$ & $100 \%$ & $100 \%$ & $57.7 \%$ & $99.2 \%$ & $100 \%$ & $100 \%$ \\
\hline 1.200 & $81.0 \%$ & $100 \%$ & $100 \%$ & $100 \%$ & $77.5 \%$ & $100 \%$ & $100 \%$ & $100 \%$ \\
\hline 1.300 & $97.8 \%$ & $100 \%$ & $100 \%$ & $100 \%$ & $97.6 \%$ & $100 \%$ & $100 \%$ & $100 \%$ \\
\hline 1.400 & $99.9 \%$ & $100 \%$ & $100 \%$ & $100 \%$ & $99.9 \%$ & $100 \%$ & $100 \%$ & $100 \%$ \\
\hline 1.500 & $100 \%$ & $100 \%$ & $100 \%$ & $100 \%$ & $100 \%$ & $100 \%$ & $100 \%$ & $100 \%$ \\
\hline 1.600 & $100 \%$ & $100 \%$ & $100 \%$ & $100 \%$ & $100 \%$ & $100 \%$ & $100 \%$ & $100 \%$ \\
\hline 1.700 & $100 \%$ & $100 \%$ & $100 \%$ & $100 \%$ & $100 \%$ & $100 \%$ & $100 \%$ & $100 \%$ \\
\hline 1.800 & $100 \%$ & $100 \%$ & $100 \%$ & $100 \%$ & $100 \%$ & $100 \%$ & $100 \%$ & $100 \%$ \\
\hline 1.900 & $100 \%$ & $100 \%$ & $100 \%$ & $100 \%$ & $100 \%$ & $100 \%$ & $100 \%$ & $100 \%$ \\
\hline 2.000 & $100 \%$ & $100 \%$ & $100 \%$ & $100 \%$ & $100 \%$ & $100 \%$ & $100 \%$ & $100 \%$ \\
\hline
\end{tabular}

